\title{
Role of antibodies in autoimmunity of the central nervous system
}

\author{
Dissertation \\ for the award of the degree \\ "Doctor rerum naturalium" \\ of the Georg-August-Universität Göttingen
}

within the doctoral program "Molecular Biology of Cells"

of the Georg-August University School of Science (GAUSS)

submitted by

César Cordero Gómez

from Cádiz

Göttingen, 2019 



\title{
Thesis committee
}

\author{
Prof. Dr. Alexander Flügel
}

Institute for neuroimmunology and multiple sclerosis research (IMSF), University Medical Center Göttingen

Prof. Dr. Jürgen Wienands

Institute for cellular and molecular immunology. University Medical Center Göttingen

Prof. Dr. Heidi Hahn

Institute for human genetics, section of molecular developmental genetics. University Medical Center Göttingen

\section{Members of the examination board}

Referee: Prof. Dr. Alexander Flügel, Institute for neuroimmunology and multiple sclerosis research (IMSF), University Medical Center Göttingen

$2^{\text {nd }}$ Referee: Prof. Dr. Wienands, Institute for cellular and molecular immunology. University Medical Center Göttingen

\section{Further members of the examination board}

Prof. Dr. Heidi Hahn, Institute for human genetics, section of molecular developmental genetics. University Medical Center Göttingen

Prof. Dr. Wolgang Brück

Department of Neuropathology, University Medical Center Göttingen

Prof. Dr. Stadelmann-Nessler

Department of Neuropathology, University Medical Center Göttingen

Prof. Dr. mult. Thomas Meyer

Laboratory for molecular psychocardiology, University Medical Center Göttingen 
Declaration

I hereby declare that I have written the PhD thesis entitled "Role of antibodies in autoimmunity of the CNS" on my own with no other sources and aids than quoted. This thesis has not been submitted elsewhere for any academic degree.

\section{César Cordero Gómez}

Göttingen, September 2019 
Table of contents

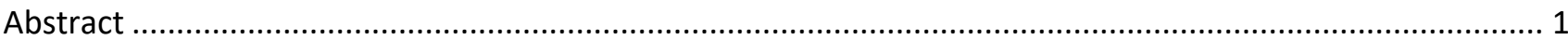

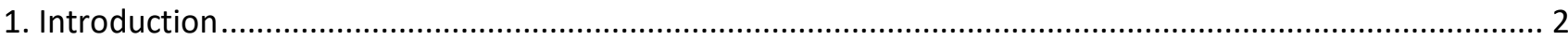

1.1. Multiple sclerosis as a heterogeneous immune-mediated disease ...................................................... 2

1.2. Animal models for the study of Multiple Sclerosis: Experimental Autoimmune Encephalomyelitis........... 3

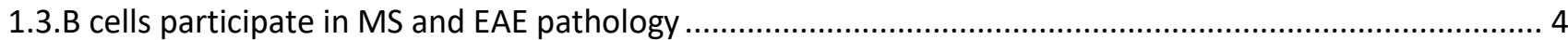

1.4. B cell activation leads to the production of different antibody isotypes through Class-switch

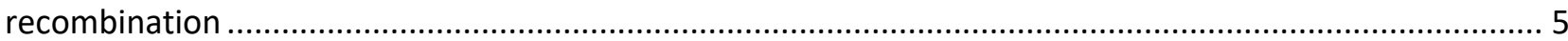

1.4.1. BCR activation triggers antigen loading into MHC-II and exposure at the $\mathrm{B}$ cell membrane............... 5

1.4.2. Class switch recombination is crucial for the maturation of the antibody response ......................... 7

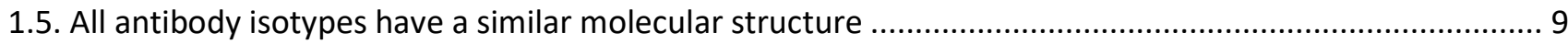

1.6. Antibody isotypes have different properties and biological functions ............................................... 10

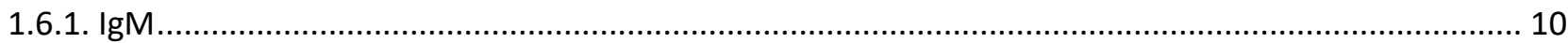

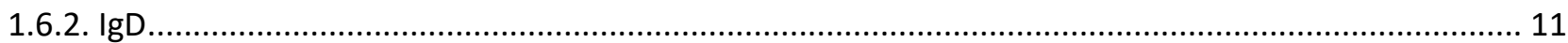

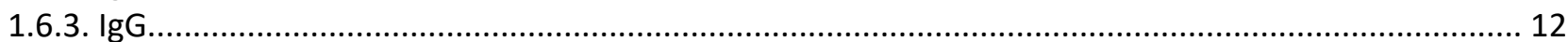

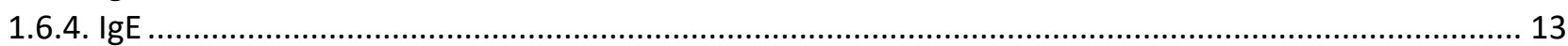

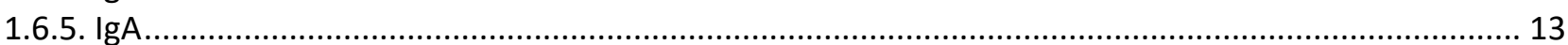

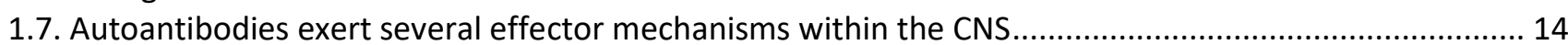

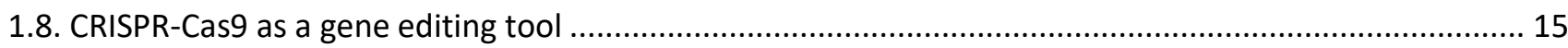

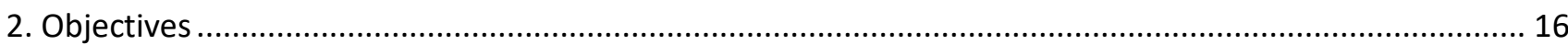

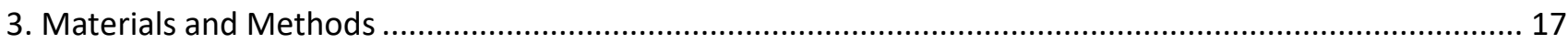

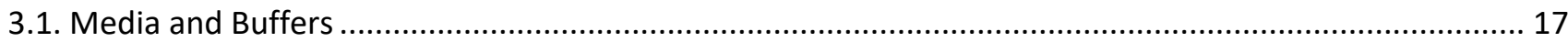

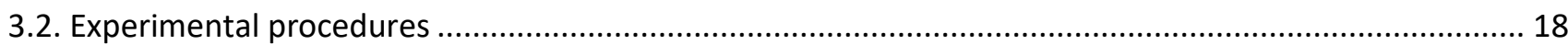

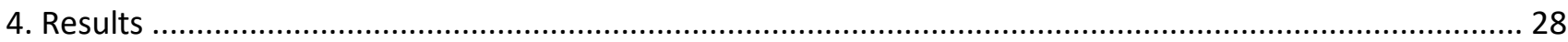

4.1. CRISPR-Cas9-driven isotype switch enables the generation of MOG specific antibodies with different

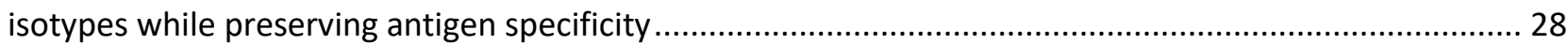

4.1.1. 8.18c5 hybridoma cells produce exclusively MOG-specific IgG1 antibodies ................................... 28

4.1.2. Design and cloning strategy of a CRISPR-Cas9 based approach for the induction of antibody isotype

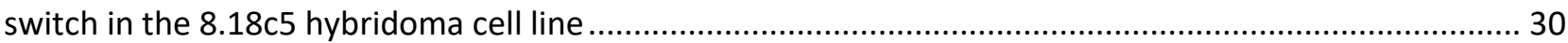

4.1.3. High cleavage efficiency of the target sequence is achieved with specific guide RNAs .................... 33

4.1.4. CRISPR-Cas9 targeting upstream the IgH constant regions of IgG1 and IgE lead to isotype switch in

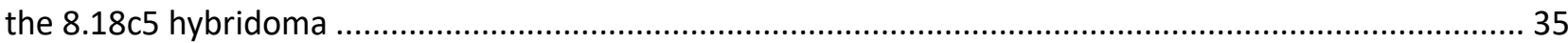

4.1.5. Selected isotype switched hybridoma clones release MOG-specific antibodies of the desired isotype

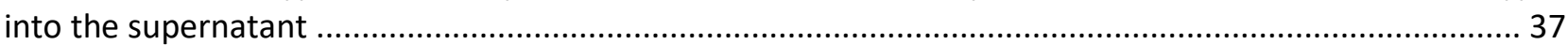

4.1.6. CRISPR-Cas9 allows the generation of different antibody isotypes in the Z2 hybridoma ................. 39

4.2. The antibody isotype determines different clinical outcomes in the EAE model .................................. 42

4.2.1. MOG-specific $\lg G 1$ and $\lg G 2 b$ accelerate the disease onset and enhance disease severity in the EAE

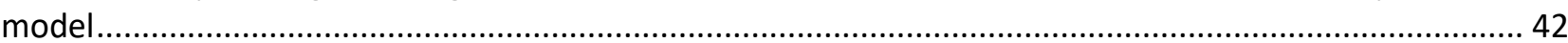

4.2.2. Disease modulatory effects on EAE mediated by MOG-specific $Z 2$ antibodies are also isotype

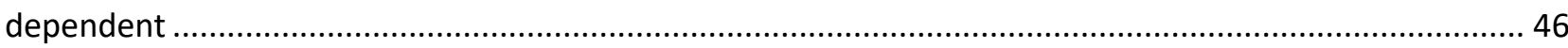

4.3. 8.18c5 IgG1 re-activate more efficiently infiltrating $T$ cells than other antibody isotypes at the onset of EAE.

4.4. Antibody isotype modify CNS demyelination and macrophage recruitment to the CNS in EAE .............. 50 
4.4.1. Antibody-driven CNS demyelination is dose- and isotype-dependent

4.4.2. Macrophage and T cell infiltration is not different upon administration of low doses of different

$8.18 \mathrm{c} 5$ or $\mathrm{Z2}$ antibody isotypes

4.4.3. Intrathecal administration of low doses of 8.18c5 antibody isotypes also enhance CNS demyelination.

4.4.4. Administration of 8.18c5 antibody isotypes at the peak of EAE does not lead to enhanced demyelination.

4.5. Antibody-dependent effector mechanisms within the CNS are dependent on Fc receptors expression . 60 4.5.1. MOG-specific IgG1 autoantibodies enhance CNS demyelination in Fcy receptor knock out animals but do not accelerate EAE onset

4.5.2. Enhanced demyelination in FcyRI-III-IV knock out animals is isotype dependent ..............................63

4.6. Enzymatic cleavage of antibody glycosylation pattern modify clinical outcome of EAE ...................... 65

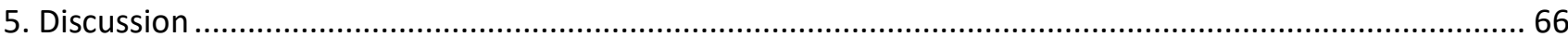

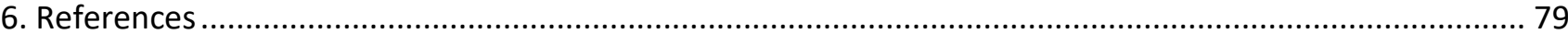


Abbreviations

$A b$

ACK

ADCC

ADCC

ADEM

AID

APC

APP

APS

ASC

BBB

$\mathrm{BCR}$

Cas

CDC

CDCC

CDCP

CFA

$\mathrm{C}_{\mathrm{H}}$

CLIP

CNS

CRISPR

crRNA
Antibody

Ammonium-Chloride-Potassium

Antibody-dependent cellular cytotoxicity

Antibody-dependent cell cytotoxicity

Acute disseminated encephalomyelitis

Activation-induced cytidine deaminase

Antigen-presenting cells

Amyloid precursor protein

Ammonium persulfate

Antibody-secreting cell

Blood brain barrier

B cell receptor

CRISPR-associated

Complement-dependent cytotoxicity

Complement-dependent cell cytotoxicity

Complement-dependent cell phagocytosis

Complete Freund's adjuvant

Heavy chain constant region

Class II-associated invariant chain peptide

Central nervous system

Clustered regularly interspaced short palindromic repeats

CRISPR RNA 


\begin{tabular}{|c|c|}
\hline CSF & Cerebrospinal fluid \\
\hline CSR & Class switch recombination \\
\hline ctrl & Control \\
\hline DCs & Dendritic cells \\
\hline DSB & Double strand break \\
\hline EAE & Experimental autoimmune encephalomyelitis \\
\hline EDTA & Ethylenediaminetetraacetic acid tetrasodium salt hydrate \\
\hline $\mathrm{EH}$ & Eagle's HEPES \\
\hline ELISA & Enzyme-linked immunosorbent assay \\
\hline $\mathrm{Fab}$ & Fragment antigen binding \\
\hline FACS & Fluorescent activated cell sorting \\
\hline FBS & Fetal bovine serum \\
\hline Fc & Crystallizable fragment \\
\hline FcRn & Fc receptor neonatal \\
\hline FcpR & Fc gamma receptor \\
\hline gDNA & genomic DNA \\
\hline GFP & Green fluorescent protein \\
\hline GM-CSF & Granulocyte-macrophage colony-stimulating factor \\
\hline gRNA & guide RNA \\
\hline $\mathrm{H}$ & Heavy \\
\hline $\mathrm{HITI}$ & homology-independent targeted integration \\
\hline HPRT & Hypoxanthine phosphoribosyltransferase 1 \\
\hline i.p & Intraperitoneal \\
\hline
\end{tabular}




\begin{tabular}{|c|c|}
\hline i.t. & Intrathecal \\
\hline i.v. & Intravenous \\
\hline IFNY & Interferon gamma \\
\hline $\lg$ & Immunoglobulin \\
\hline $\lg A$ & Immunoglobulin A \\
\hline $\lg D$ & Immunoglobulin D \\
\hline $\operatorname{lgE}$ & Immunoglobulin E \\
\hline $\lg G$ & Immunoglobulin G \\
\hline $\lg M$ & Immunoglobulin M \\
\hline IL & Interleukin \\
\hline INF- $\gamma$ & Interferon gamma \\
\hline ITAM & Immunoreceptor tyrosine-based activation motif \\
\hline ITIM & Immunoreceptor tyrosine-based inhibitory motif \\
\hline KO & Knock out \\
\hline $\mathrm{L}$ & Light \\
\hline L & Liters (in methods) \\
\hline LFB & Luxol fast blue \\
\hline MAC & Membrane attack complex \\
\hline MAG & Myelin associated glycoprotein \\
\hline MBP & Myelin basic protein \\
\hline $\mathrm{MHC}$ & Major histocompatibility complex \\
\hline $\min$ & Minutes \\
\hline $\mathrm{ml}$ & Milliliter \\
\hline
\end{tabular}




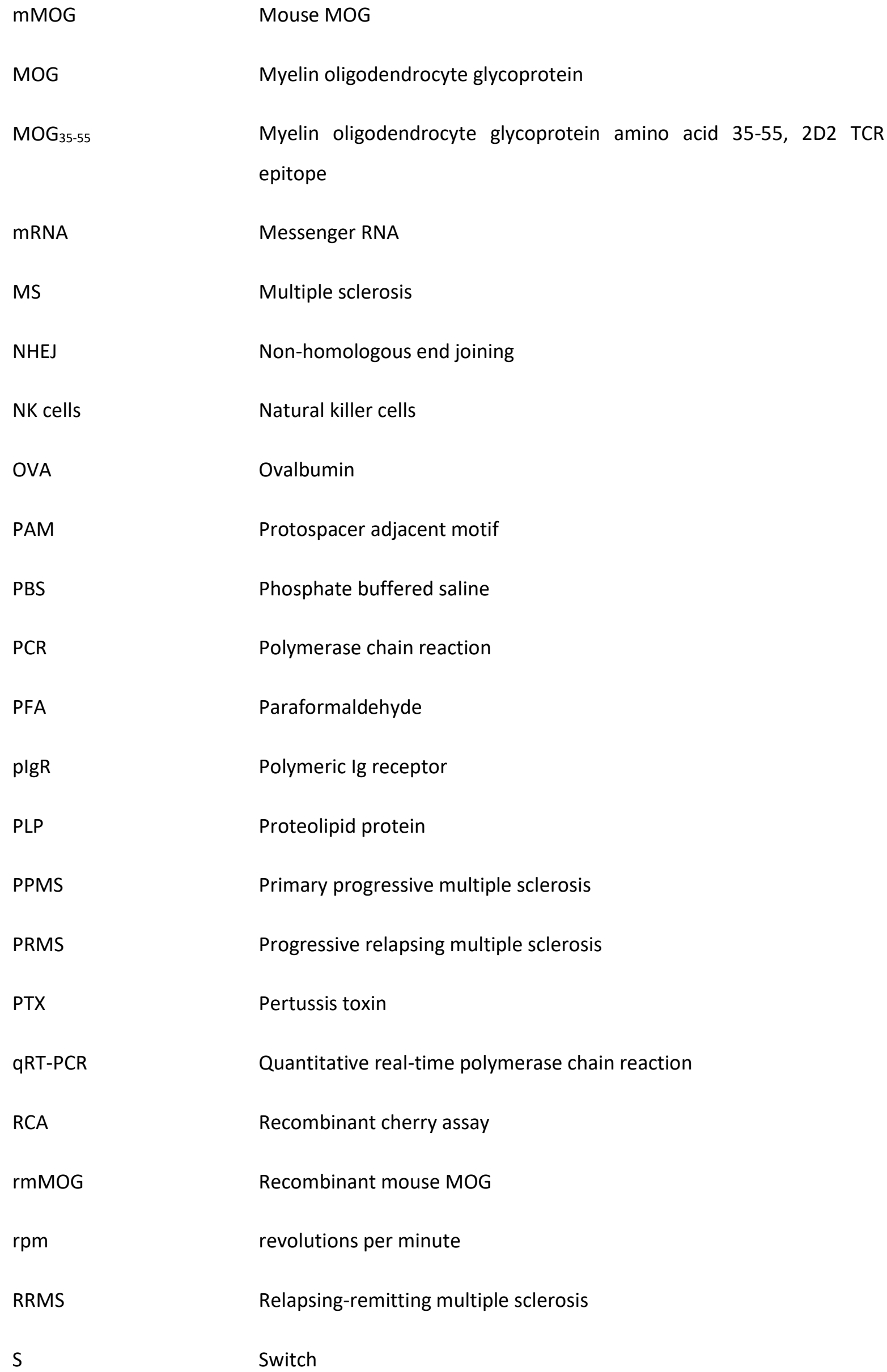


S.c.

SC

SDS-PAGE

SEM

sgRNA

slgM

SPMS

TBS

TCM

TCR

TMB

T-mMOG

tracrRNA

VDJ

WT
Subcutaneous

Spinal cord

Sodium dodecyl sulfate polyacrylamide gel electrophoresis

Standard error of the mean

Single guide RNA

Secretory lgM

Secondary progressive multiple sclerosis

Tris buffered saline

T cell medium

T-cell receptor

3,3', 5,5'tetramethyilbenzidine

Tomato labelled mouse MOG

Trans-activating RNA

Variable-Diversity-Joining

Wildtype 


\section{Abstract}

Although T cells are the main players in multiple sclerosis (MS) and its animal model experimental autoimmune encephalomyelitis (EAE), the role of $B$ cells and their soluble effector molecules (antibodies) is being progressively appreciated regarding disease development. Previous observations from our department showed that autoantigen-specific B cells contribute substantially to the pathogenesis of CNS autoimmune disease through the production of myelin-specific antibodies. These autoantibodies accumulated in CNSresident antigen-presenting cells and facilitated the reactivation of invading autoreactive T cells, resulting in an earlier manifestation of clinical disease. These results showed that antibodies exert additional mechanisms involved in CNS autoimmune disease exacerbation, in contrast to previous studies that focused exclusively on the role of antibody-mediated demyelination in EAE exacerbation. It is known that antibody effector mechanisms are dependent on the antibody isotype. Therefore, the aim of this study was to modify the antibody isotype produced by myelin-specific hybridoma cell lines and to characterize the mechanisms by which antibodies of different isotypes exert their pathogenic functions in CNS autoimmunity. Using genetic engineering approaches based on the recently characterized CRISPR-Cas9 system, we could produce different isotypes from two hybridoma cell lines, which produce antibodies specific for myelin oligodendrocyte glycoprotein (MOG), while preserving their antigen specificity. We could demonstrate that antibody diseasemodifying effects were isotype-dependent in the active and transfer EAE model, with all investigated IgGs subclasses but no any other antibody isotypes tested being involved in disease acceleration. Furthermore, using a T-cell activation reporter (Nur 77-GFP) we found that IgG-treated animals presented higher percentages of activated CD4+ T cells in the CNS. Regarding the role of demyelination in EAE, our histological analysis indicated that exclusively antibodies of the IgG isotype had demyelinating potential, with IgG2a and $\lg 2 \mathrm{~b}$ antibodies showing more demyelination compared to IgG1. Moreover, in the absence of Fcy receptors (FcyR I-IV KO mice), the disease acceleration effect was abrogated but, interestingly, the demyelinating potential of antibodies of the IgG isotype was increased. Taken these results into consideration, we propose that the disease acceleration effect and CNS demyelination are two independent mechanisms driven by antibodies. While disease acceleration is dependent on the presence of Fc receptors, demyelination seems to be dependent on other molecular cues, such as the complement system. Antibody isotypes have different affinities regarding Fc receptor binding and complement fixation and, consequently, there is a preference towards one of these systems. However, the preferential effector mechanism triggered by these antibody isotypes can be altered depending on the presence, or absence, of the required molecular cues. 


\section{Introduction}

\subsection{Multiple sclerosis as a heterogeneous immune-mediated disease}

Multiple sclerosis (MS) is the most common chronic, inflammatory demyelinating disease of the central nervous system $(\mathrm{CNS})^{1,2}$. Among young adults, it represents the main cause of neurologic disability, affecting over 2.5 million individuals worldwide. MS pathological hallmarks are classically found in the brain and, in some patients, also in the spinal cord. These lesions are the consequence of an autoimmune attack of autoreactive immune cells to the myelin sheaths, leading to tissue inflammation, demyelination, axonal damage and gliosis ${ }^{3}$.

There are two major forms of MS, Relapsing-remitting MS (RRMS) and Primary Progressive MS (PPMS), which represent $85-90 \%$ and $10-15 \%$ of the patients respectively ${ }^{1}$. RRMS affects about two times more frequently women than men, and it is characterized by recurrent episodes of neurologic dysfunction followed by partial recovery. Approximately $60 \%$ of the RRMS patients develop later in life a secondary progressive (SPMS) disease course, which may lead to disease exacerbation associated with neurodegeneration and progressive accumulation of disability. PPMS patients, representing the smallest fraction of MS patients, undergo a sudden disease onset and constant disease progression. Additionally, a rare variant of PPMS presents acute exacerbations with periods of relapse (progressive-relapsing MS) ${ }^{1,3}$.

The histopathological alterations among patients, as well as the affected CNS functions and response to treatment, can vary significantly, indicating that MS is a highly heterogeneous disease specially at early timepoints ${ }^{4}$. Even though the factors determining this heterogeneity are not completely understood, it is probably a consequence of the interplay between both etiological risk factors and pathomechanisms ${ }^{4,5}$. The etiology of MS involves the interaction of environmental factors and several complex genetic traits. Environmental factors participating in MS etiology include low vitamin D levels ${ }^{6,7}$, diet habits such as high salt intake and smoking ${ }^{8,9}$, but also infections such as Epstein-Barr virus ${ }^{5}$. Concerning the genetic traits promoting MS, genome-wide association studies have identified several risk loci, almost all of them related to the immune system ${ }^{10}$. Examples of these loci are variations of the human leukocyte antigen (HLA) and genes coding for cytokine pathways. Among the most relevant pathomechanisms involved in MS etiology, autoimmune inflammation, de-and remyelination, secondary and primary neurodegeneration with axonal and neuronal loss, astrogliosis and metabolic alterations are included ${ }^{1,3,11}$. All these pathomechanisms interplay in diverse manners on different groups of patients, being this interplay probably underlying the different phenotypical presentations of MS. 
Although MS lesions can arise anywhere in the CNS, there is a preferential distribution in the optic nerve, spinal cord, brain stem and periventricular areas ${ }^{2}$. White matter pathology is a common feature of MS lesions, but grey matter pathology can arise in areas like cortex, deep grey matter and spinal cord, being commonly associated with the chronic phase of the disease ${ }^{1,2}$. Autopsies and biopsies from patients allowed the definition of four lesion patterns based on infiltrating immune cells, deposition of humoral factors, and loss of oligodendrocyte and/or myelin proteins. Demyelinating lesions of pattern I and II share similar features, where predominantly macrophages, but also T cells, are present surrounding active demyelination plaques close to veins and venules. These two patterns are distinguished by the deposition of Igs and complement C9neo antigen at the demyelination sites, where C9neo antigen deposition is exclusive to pattern II. Pattern III lesions also contain inflammatory infiltrates composed of mainly $T$ cells, but also macrophages and activated microglia. The main characteristic of pattern III lesions is the loss of myelin-associated glycoprotein (MAG) and oligodendrocyte apoptosis, which can sometimes extend into to the normal appearing white matter. Finally, pattern IV lesions are also characterized by presence of large amounts of T cells and macrophages, primary oligodendrocyte degeneration and lack of remyelination plaques.

\subsection{Animal models for the study of multiple sclerosis: experimental autoimmune encephalomyelitis}

Experimental Autoimmune Encephalomyelitis (EAE) is the most widely used model for the study of $\mathrm{MS}^{11}$. Despite not being the only model for the study of MS, EAE has made an important contribution to the understanding of MS since its establishment during the $20^{\text {th }}$ century ${ }^{12}$. Several animal species have been used for the induction of EAE, rodents being nowadays the most frequently used ones ${ }^{13}$, though non-human primates are also commonly used, given their close evolutionary relationship with humans ${ }^{11}$.

In rodents, EAE can be induced both by active immunization with CNS antigens or by adoptive transfer of myelin specific T cells. In the active EAE model, animals are immunized with an emulsion containing complete Freund's adjuvant (CFA), and myelin components with myelin oligodendrocyte glycoprotein (MOG), myelin basic protein (MPB), proteolipid protein (PLP) or with its T-cell relevant peptides as the most frequent examples. This immunization protocol induces the activation and proliferation of myelin-specific T cells in draining lymph nodes, which egress from the lymph nodes to the bloodstream and are able, in a later stage, to invade the CNS. T-cells infiltration into the CNS occurs together with other cells of the immune system, such as neutrophils, monocytes, macrophages, dendritic cells and NK cells. All these cells together mediate lesion formation and demyelination, producing a MS-like clinical course characterized by ascending paralysis and weight loss ${ }^{11}$. In the adoptive transfer EAE model, pre-activated myelin-specific $T$ cells are transferred into recipient animals, which develop an ascending paralysis during the following days after T-cell transfer ${ }^{14,15}$. These activated myelin-specific T cells do not require further priming in the lymph nodes to invade the CNS. 
However, CNS-invading T cells in both the active immunization protocol and the adoptive transfer protocol require a further reactivation step at the meninges by local antigen-presenting cells ${ }^{16,17}$.

Both EAE and MS are driven by autoimmune processes, even though the animal model does not fully resemble all the features of human MS. Furthermore, while in MS animal models the antigen is known, this is not the case for MS. However, the development of different EAE models by using different antigens and mouse strains allowed the reproduction of different aspects of MS.

For example, a chronic form of EAE where lesions are predominantly present in the spinal cord but not in the brain can be induced upon immunization of $\mathrm{C} 57 \mathrm{BI} / 6$ mice with MOG protein or the T cell epitope MOG $_{35-55}{ }^{3,12,13}$. Furthermore, MOG-specific $T$ cell receptor transgenic mice (2D2 mice) develop spontaneous autoimmune disease with a very low incidence (4\%), whereas double transgenic animals presenting MOG-specific T and B cells develop spontaneous disease with an incidence of $58 \%{ }^{18}$. Moreover, the relapsing-remitting course of MS can be modelled by immunization of SJL mice with PLP, or by adoptive transfer of PLP ${ }_{139-151}$ primed T cells ${ }^{19,20}$. Finally, T-cell receptor transgenic mice on the SJL background can spontaneously develop EAE with a relapse-remitting disease course ${ }^{21}$. Nowadays, only few animal models are available that can reproduce the brain inflammation observed in MS patients ${ }^{22,23}$. In this regard, our department recently developed a new model in the Lewis rat by adoptive transfer of $\beta$-synuclein specific T cells, which resembled several pathological hallmarks of MS, including grey matter inflammation and brain atrophy ${ }^{24}$.

\subsection{B cells participate in MS and EAE pathology}

Although $T$ cells are the main drivers of MS, the role of $B$ cells in disease pathogenesis is becoming progressively more acknowledged. The hypothesis of B-cell contribution to MS pathogenesis is supported by the presence of oligoclonal bands in the CSF of MS patients ${ }^{25,26}$, the beneficial effects observed in patients undergoing B-cell depleting therapies with monoclonal anti-CD20 antibodies ${ }^{27,28}$, and the therapeutic outcome of plasma exchange ${ }^{29}$. Furthermore, the deposition of complement and immunoglobulins within the CNS parenchyma as well as the presence of tertiary lymphoid organs within the meningeal tissue of MS patients supports the crucial role of $B$ cells in MS pathology ${ }^{30,31}$. In the EAE model, B cells have been reported to have disease-modifying effects, both ameliorating and worsening the disease. Knock in C57BI/6 animals carrying a $B$ cell receptor (BCR) with restricted specificity to MOG (Th mice), develop a more severe clinical course after immunization with recombinant MOG protein ( $r m M O G$ ) compared to Wild-type (WT) animals ${ }^{32}$. In spite of the restricted MOG-specific BCR expression in these animals, they do not develop spontaneously neurological deficits, but nevertheless, when Th mice are crossed with transgenic animals carrying $T$ cells with restricted TCR specificity to MOG (2D2 mice), 58\% of the offspring develop spontaneous EAE $^{33}$. 
Extensive research on the EAE model has also identified B cells as important players in CNS inflammation through different pathogenic mechanisms. Molnarfi and collaborators showed that antigen presentation represents a crucial mechanism for disease promotion, given that selective knock out of MHC II expression on $B$ cells led to disease resistance ${ }^{34}$. Furthermore, B cell depletion therapy also ameliorates EAE disease course, presumably by the reduction of pro-inflammatory cytokines such as IL-6, and consequently leading to a general reduction of bystander activation of immune cells ${ }^{35}$, although other studies have pointed at $B$ cells driving antiinflammatory effects in EAE through the production of cytokines such as IL-10 or IL-35 ${ }^{36,37}$. Finally, activated self-reactive B cells can differentiate into plasma cells, which may produce antibodies recognizing CNS structures and therefore exacerbating the damage in CNS lesions ${ }^{38}$.

\subsection{B cell activation leads to the production of different antibody isotypes through Class-switch recombination}

\subsubsection{BCR activation triggers antigen loading into MHC-II and exposure at the $B$ cell membrane}

Immature B cells are generated in the bone marrow, and they are selected through an assessment of the functionality of their $\mathrm{BCR}^{39}$. After selection, these immature $\mathrm{B}$ cells migrate to the spleen to undergo their final maturation stages, becoming naïve mature B cells. Upon activation, they differentiate into plasmablasts (short lived) and plasma cells (long lived), which are antibody-secreting cells (ASC).

Antigen binding to the BCR triggers B cell activation, where a signalling cascade mediated by cytoplasmic immunoreceptor tyrosine activations motifs (ITAMs), and several tyrosine kinases such as Lyn and Syk, mediate the internalization of the BCR-antigen complex in a clathrin-dependent manner ${ }^{40}$. The formed vesicles then undergo a series of biochemical changes evolving to a late endosome and, in a later stage, they are delivered to peptide-loading compartments, which contain newly synthesized MHC-II molecules plus the invariant chain (li) (Figure 1). li contains the Class-II associated invariant chain peptide (CLIP), which prevents the binding of self-peptide fragments to MHC-II during this process. The $\mathrm{pH}$ of these compartments decreases progressively, allowing Ig processing into antigen-binding $(\mathrm{Fab})$ and crystallizable fragment $(\mathrm{Fc})$ and, after a cascade of folding and proteolytic events, the antigen becomes available. Processing of li by DM upon acidic degradation of DO (which constitutively inhibits DM), allows the peptides to be loaded in MHC-II molecules, which are then transported to the cell membrane in order to be exposed. 


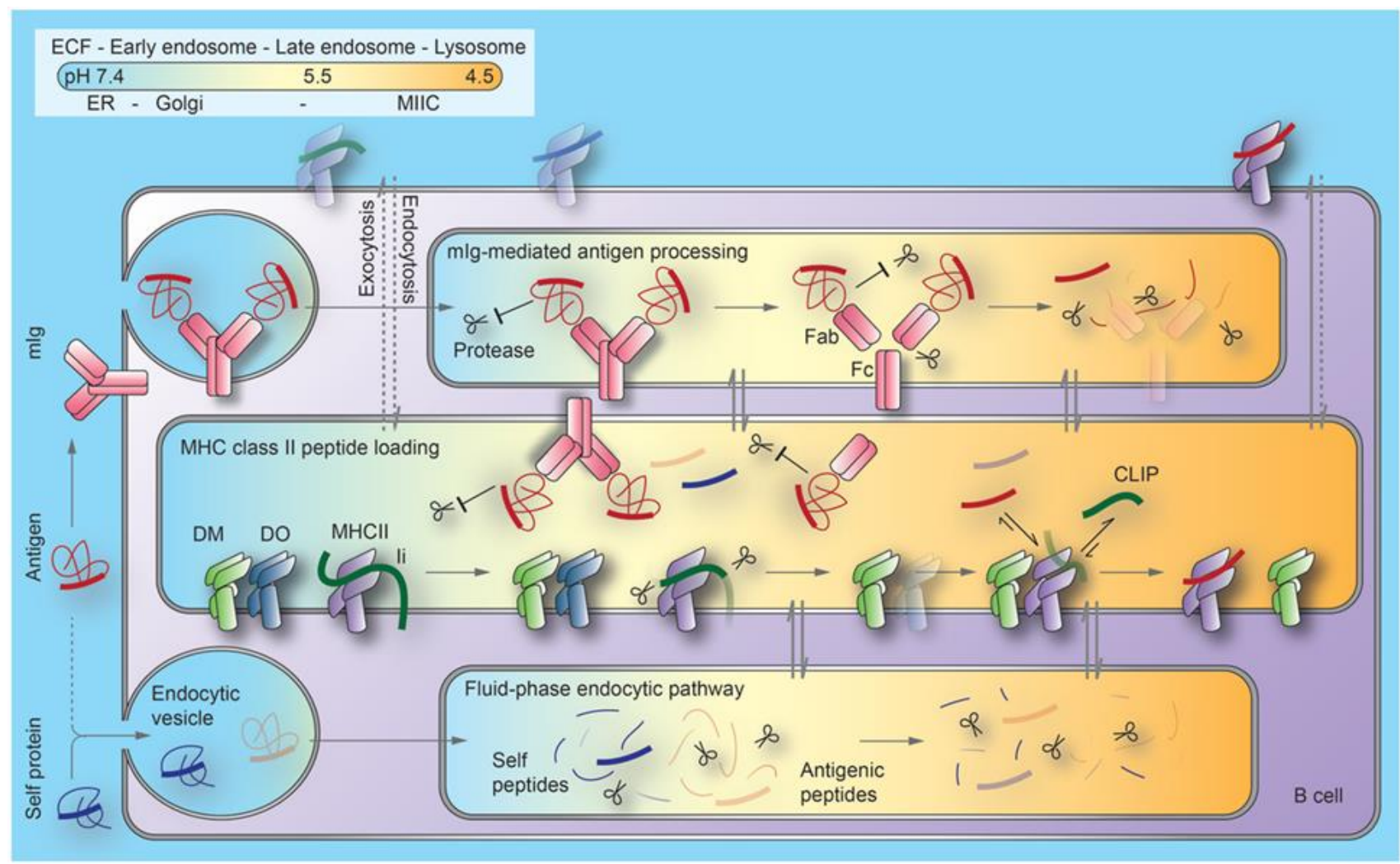

Figure 1. B cell activation by crosslinking of the BCR with its specific antigen trigger internalization of the BCR-antigen complex and antigen loading into MHC-II molecules. A schematic view of the events after BCR activation is depicted. Crosslinking of the antigen with the BCR elicits its internalization and processing. Proteolytic cleavage of the Fab and Fc Ig fragments makes the antigen available to be loaded in MHC-II molecules. Newly formed MHC-II molecules covered by $\mathrm{li}$ are processed, conforming the endosomal $\mathrm{pH}$ increase, leading finally to free MHC-II molecules that can bind the internalized antigen, and be presented at the cell membrane for its recognition by CD4+ T cells. Adapted from Adler et al. (2017) Front Immunol.

In the peripheral lymphoid organs, $\mathrm{CD} 4+\mathrm{T}$ helper cells recognize their cognate antigens presented by activated B cells in the context of MHC-II and coordinate their differentiation into antibody-secreting cells ${ }^{41,42}$. These T helper cells express CD40 ligand upon TCR engagement, which binds to CD40 expressed at the surface of B cells. Further signals are required to activate $B$ cells, such as interaction of B cell CD80/CD86 with CD28 expressed by T cells, and release of cytokines by CD4+ T cells such as IL-4, IL-5 and IL-6. Additionally, ligation of CD40 to CD40L synergizes with IL-4 to induce the expression of the activation-induced cytidine deaminase (AID) protein ${ }^{43,44}$. All these events together trigger B cell proliferation, somatic hypermutation and Class-switch recombination (CSR). Somatic hypermutation generates a high amount of point mutations in the $V$ genes of the heavy $(\mathrm{H})$ and light $(\mathrm{L})$ chains, which lead to the generation of high-affinity antibodies against a concrete antigen. On the other hand, CSR modifies the immunoglobulin heavy-chain constant region $\left(\mathrm{C}_{H}\right)$ gene expressed from the constant $\mu$ region to a different $C_{H}$ genes, resulting in a switch of the immunoglobulin isotype from IgM/IgD to IgG, IgE or IgA while preserving antigen specificity ${ }^{42}$. 


\subsubsection{Class switch recombination is crucial for the maturation of the antibody response}

The immunoglobulin constant $\mathrm{H}$ locus consists of an ordered array of constant $\mathrm{H}$ genes, which are flanked at their $5^{\prime}$ region by a switch $(S)$ region composed of tandemly repetitive unit sequences with several palindromic sequences $^{43}$ (Figure 2). CSR takes place between two $S$ regions located upstream of the constant $\mathrm{H}$ of $\mathrm{C} \mu$ and a different constant domain located downstream of $C \delta$. The $C \mu$ gene is located downstream of the $V_{H}(D) J_{H}$ cassette ${ }^{43,45}$. Consequently, $C S R$ between $S \mu$ and another $S_{H}$ region located $5^{\prime}$ to a $C_{H}$ gene brings that particular $C_{H}$ gene adjacent to the $V_{H}(D) J_{H}$ cassette exon. Three steps drive class-switch recombination: (i) choice of a downstream $\mathrm{S}$ region as a target to pair in a later stage with the targeted $\mathrm{S} \mu$ region, (ii) recognition and cleavage of the target DNA by AID, and (iii) repair and ligation of the broken DNA ends by non-homologous end joining (NHEJ), resulting in a looped-out deletion. CSR is highly dependent on cell proliferation, which is related to the ability of the cell to switch to a different isotype. Two complete rounds of cell division are required to switch to $\lg G$ and $\operatorname{IgA}$, and probably additional rounds are require for switching to $\operatorname{IgE}{ }^{42,43}$. $C S R$ towards a concrete $C_{H}$ domain is dependent on the cytokine production by CD4+ T cells. For example, while isotype switching to IgA is highly dependent on TGFB1, IL-4 dependent activation of STAT6 is required for switching to IgE. Further immune products participate in the polarization of CSR towards a concrete antibody isotype ${ }^{46,47}$. 
a Heavy chain genes in IgM-expressing cells

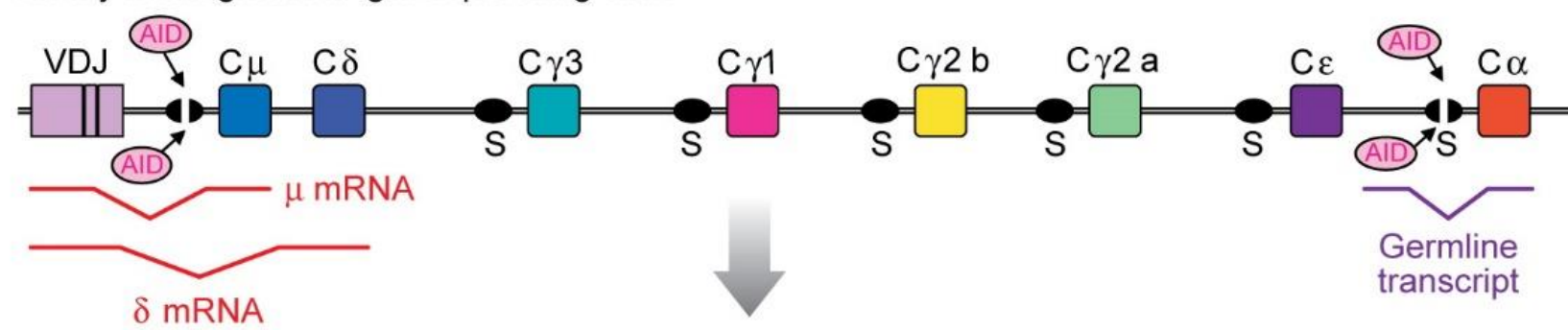

b Switch recombination (requires DSBs)

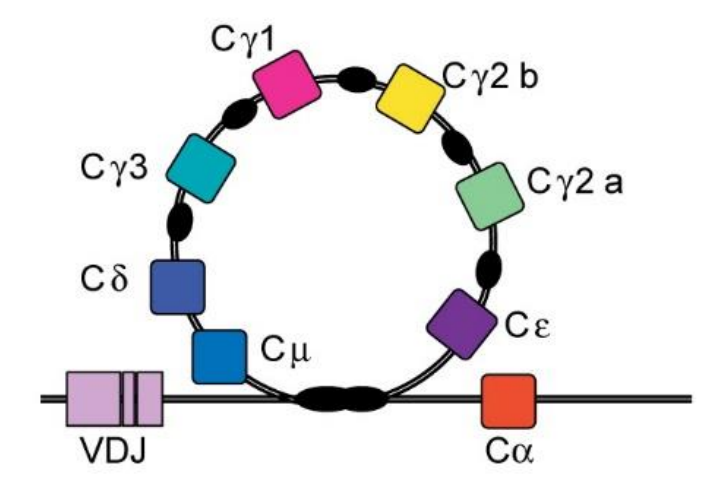

C Heavy chain genes
in IgA-expressing cell

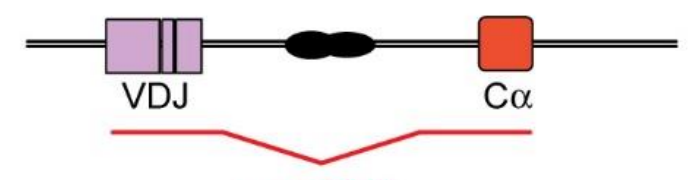

$\alpha$ mRNA

Figure 2. Immunoglobulin isotype class switch is driven by an intrachromosomal deletional recombination event. An example of Ig class switch recombination is depicted. Upon ligation of CD40-CD40L, transcription of AID is induced. This enzyme deaminates $\mathrm{dC}$ resdiues in both sides of transcriptionally active $\mathrm{S}$ regions, which flank the constant $\mathrm{H}$ sequences for different isotypes at their $5^{\prime}$ side, and starting a process that leads to double-strand DNA breaks in both S regions. Ligation of both opened $\mathrm{S}$ regions by NHEJ repair mechanisms leads to the positioning of a new constant $\mathrm{H}$ region adjacent to the $\mathrm{VH}$ exon. Transcription of this locus lead to production of antibodies of a different isotype. Adapted from Stavnezer et al (2009). Annu Rev Immunol. 


\subsection{All antibody isotypes have a similar molecular structure}

Immunoglobulins belong to the immunoglobulin super-family, which is a large family of cell surface and soluble proteins involved in the recognition, binding, or adhesion of cells. Members of this superfamily have structural features in common with immunoglobulins.

Antibodies consist of two heavy and two light chains (Figure 3). The light chains can be further classified as either the kappa or the lambda chain based on differences in polypeptide sequence. The antibody structure contains two antigen-binding fragments (Fabs) and the crystallisable fragment (Fc), which are linked together through a disulphide bridge between two conserved cysteine residues at the heavy chains, and each heavy chain is linked to a light chain by a disulfide bond ${ }^{48}$. In every immunoglobulin molecule, the two heavy and light chains are identical, making it possible for antibodies to bind simultaneously two identical structures ${ }^{49}$.

While the light chains contains one variable domain and one constant domain, the heavy chains contain one variable domain, and either three (IgD, IgG and $\operatorname{IgA}$ ) or four constant domains (IgM and IgE). A spacer hinge region between the first and second constant domains $(\mathrm{CH} 1$ and $\mathrm{CH} 2)$ is normally present in immunoglobulins with three $\mathrm{C}$ domains. The class, and therefore the effector function of an antibody, is defined by the structure of its heavy chain. The five major classes of immunoglobulin are IgM, IgD, IgG, IgA and IgE. These isotypes differ in their abundance in plasma, preferential location, half-life and biological functions. IgGs represent the most abundant antibody isotype in mouse serum ${ }^{49,50}$.

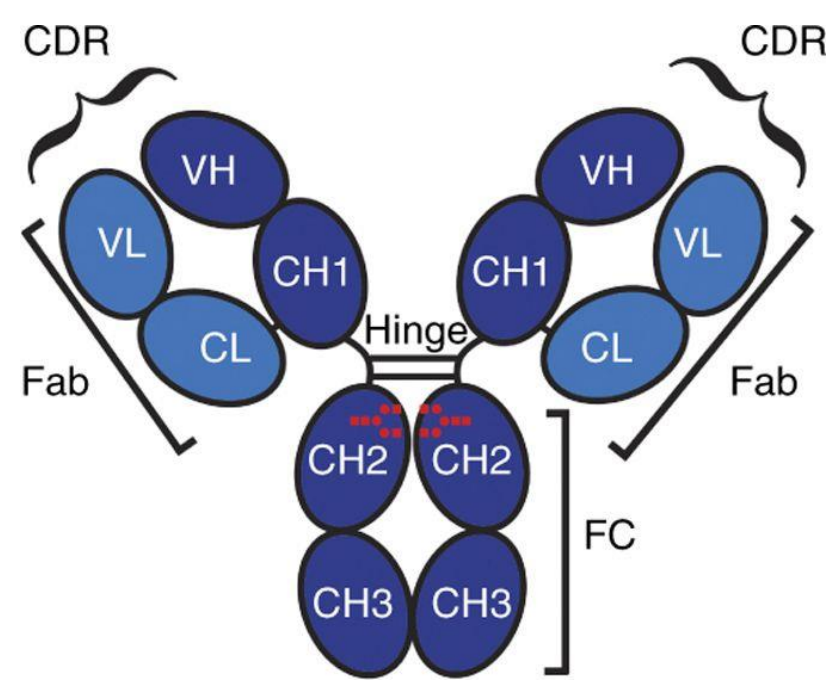

Figure 3. Antibody structure consists of two heavy chains and two light chains. Antibody structure of a regular lgG molecule is depicted. Two heavy chains (dark blue) are connected to each other by a disulphide bridge. The light chains (light blue) are connected to the heavy chains also by disulphide bridges, configuring the Fab region. The two Fab fragments are connected to the Fc part through the hinge region, where the link of the two heavy chains occur. At the $\mathrm{CH} 2$ region of IgGs is where glycosylations are found (red dots). Adapted from Rosati et al. (2014). Nat Prot 


\subsection{Antibody isotypes have different properties and biological functions}

\subsection{1. $\lg M$}

IgM is the first antibody class produced by $\mathrm{B}$ cells during an immune response. IgM can exist as a membranebound monomer or as complex structure, where several IgM monomers form a pentamer ${ }^{51}$. In both mice and in humans, pentameric IgM has a molecular weight of $900 \mathrm{kDa}$ and high affinity to complement, having a 1000fold greater binding affinity to $\mathrm{C} 1 \mathrm{q}$ compared to IgG. A full IgM pentamer contains three different polypeptides types: heavy chains, light chains and J-chain. While the light chains are identical to other isotypes, IgM contain four constant heavy domains $(\mathrm{CH} 1-\mathrm{CH} 4)$, where the last heavy domain $(\mathrm{CH} 4)$ associate to the J-chain, allowing the binding to other IgM monomers and adopting a pentamer configuration (Figure 4).

The secreted form of IgM (slgM) has low affinity for antigens compared to other antibody isotypes, but its pentameric structure confers IgM a high valency, and therefore it is highly efficient mediating agglutination and elimination of pathogens ${ }^{52}$. Secretory $\operatorname{lgM}$ can be further classified in natural and immune $\operatorname{lgM}^{53}$. Natural IgM is a polyreactive immunoglobulin, and it drives phagocytosis of apoptotic cells through C1q binding. On the other hand, exposure to pathogens induce the production of immune $\operatorname{lgM}$, which is antigen specific. Complement binds to $\operatorname{lgM}$ when it recognizes its cognate antigen, eliciting an enhanced humoral response where the production of antigen specific lgM is increased by several hundred-fold ${ }^{54}$.

Three different types of receptors mediate the effects of IgM: Fc $\mu R$, polymeric Ig receptor (plgR) and the receptor for both $\operatorname{lgA}$ and $\operatorname{lgM}(F c \alpha / \mu R)^{55,56}$. plgR is expressed on the mucous epithelium and ducts of secretory glands, at the basolateral membrane of epithelial cells, as well as in the intestine, kidney, pancreas, lung and endometrium. This receptor mediates the transport across mucous membranes of slgA and slgM through a three-stage process, which imply the endocytosis of the complex, several enzymatic-processing steps, and finally the release of the immunoglobulin through the apical region of the epithelial cells ${ }^{52}$.

$\mathrm{Fc} \alpha / \mu \mathrm{R}$ can bind both polymeric $\lg \mathrm{A}$ and polymeric $\lg \mathrm{M}$, having higher affinity for $\lg \mathrm{M}$ than for $\operatorname{Ig} \mathrm{A}$ in mice. This receptor is expressed in all lymphoid tissues, kidney, intestine, lung, liver and myocardium. At the cell level, B cells and macrophages express Fc $\alpha / \mu R$, but not T-cells, granulocytes and NK cells. Within the intestine, Fc $\alpha / \mu R$ play a role in both local and systemic immune responses, as crosslinking with IgM and IgA on intestinal macrophages, plasma cells and Paneth cells evoke the internalization of the receptor, and this process presumably leads to antigen presentation ${ }^{52,57}$.

Fc $\mu R$ was the last described receptor for IgM. Unlike other Fc receptors, the Fc $\mu R$ does not present ITAM or ITIM motifs in its cytoplasmatic domain, but it rather contains serine and tyrosine residues susceptible to phosphorylation. This receptor is specific for IgM and does not bind other antibody isotypes, and in mice it is 
predominantly expressed by B cells, but also by monocytes, macrophages, granulocytes and dendritic cells. Despite the function of $F c \mu R$ is not yet being fully understood, recent research pinpoints to a role in regulating the transport and cell-surface expression of IgM-BCR, limiting the amount of complexes that are transported to the cell membrane ${ }^{58}$.

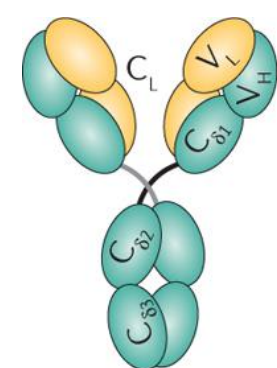

$\lg \mathrm{D}$

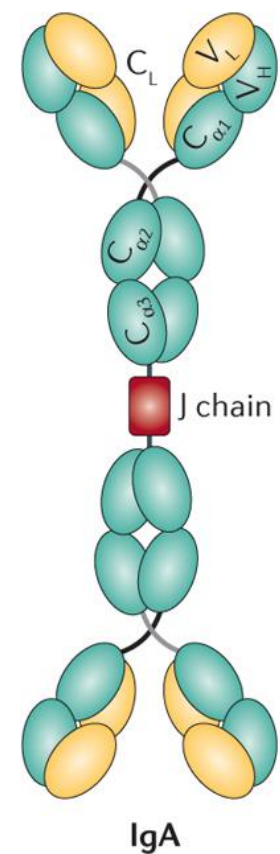

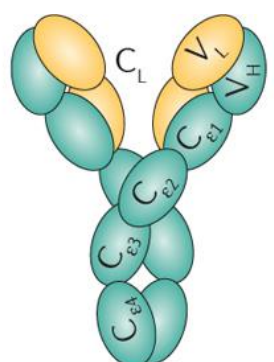

$\lg \mathrm{E}$

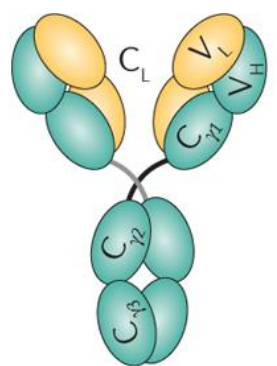

$\lg \mathrm{G}$

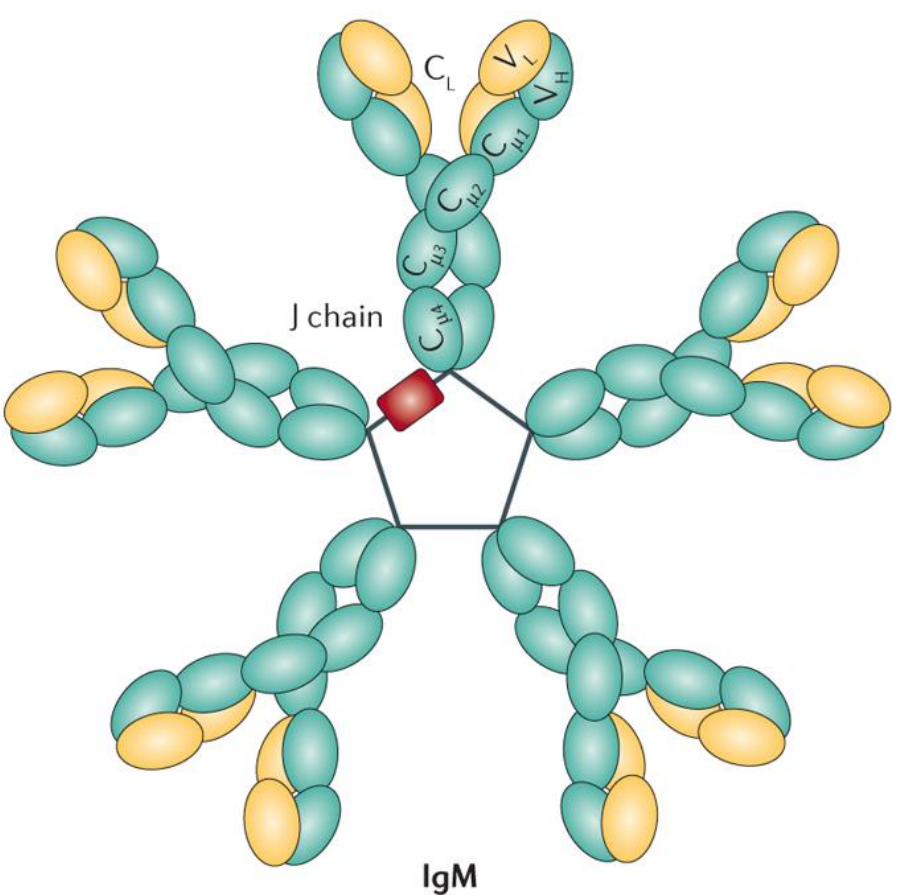

Figure 4. Antibody isotypes have different structures. The structure of the five main antibody isotypes is depicted. While IgD, IgE and IgG present a monomeric structure, IgA and IgM can form dimers by the binding of several monomers through a J-chain. Light chains are depicted in yellow. Heavy chains are depicted in blue. J-chain of IgA and IgM is indicated with a red rectangle and black lines. Adapted from Duarte JH (2016). Nat Immunol

\subsubsection{IgD}

IgD represents the most enigmatic isotype of all antibody isotypes. Its structure resembles that of IgGs, having a molecular weight of $180 \mathrm{kDa}^{59}$. Circulating $\operatorname{lgD}$ is found at very low concentrations and its half-life is rather short. It is assumed to have homeostatic functions and, although it is not known to participate in all antibody effector mechanisms, it is known to enhance antibody responses and to activate the alternative complement pathway. Antigen-naïve mature B cells dually express surface IgD and IgM, where differential polyadenylation 
and alternative splicing processing of the transcript containing both $C_{\mu}$ and $C_{\delta}$ regulate the membrane expression of both immunoglobulins. This suggest that both immunoglobulins accomplish distinct functions for relevant B cell developmental stages, although $\operatorname{lgD}$ is able to replace $\operatorname{lgM}$ in $\lg \mathrm{M}$ KO animals. Furthermore, $\mathrm{KO}$ animals for IgD develop normal immune responses but a delayed antibody affinity maturation. CD4+ T cells express IgD membrane receptors both in human and mice, which bind secreted and membrane-bound IgD and are upregulated upon cross-linking with polymeric $\lg D$ and downregulated by monomeric $\lg \mathrm{D}^{59,60}$. Finally, recent research has shown that secreted IgD binds to mast cells and basophils by interacting with the CD44 binding protein galectine-9, eliciting a Th2 cell-mediated response that enhances the production of antigenspecific $\lg G 1$ and $\lg E$ by $B$ cells ${ }^{61}$.

\subsubsection{IgG}

In humans, four IgG subclasses exist and they are classified according to their serum levels (IgG1>|gG2>IgG3>IgG4). In contrast, in mice they are classified as $\lg G 1, \lg G 2 b$, IgG2a and IgG3. The heavy chain of all these subclasses contain three constant domains, and have a molecular weight of $150 \mathrm{kDa}{ }^{49}$. They are present as monomers at high levels in serum and have a long half-life, where the intracellular neonatal Fc receptor (FcRn) plays an important role avoiding the degradation of IgGs at the lysosomal compartment ${ }^{62,63}$. The IgG subclasses have amino acid variations in the Fc domains, as well as distinct hinge regions, which make these antibody isotypes perform differently regarding their effector mechanisms. IgG1 has a higher affinity to Fc receptors and is known not to bind complement efficiently. On the other hand, IgG2b and IgG2a interact efficiently with $F c \gamma$ receptors $(F c \gamma R)$ and trigger the activation of the complement cascade with high efficiency. IgG3 is also known to bind Fc receptors with low affinity and to bind complement efficiently ${ }^{64,65}$. IgG subclass expression is influenced by the cytokine environment. While Th2 cytokines such as IL-4 induce switching to $\operatorname{IgG1}$, Th1 cytokines like IFN- $\gamma$ lead to the production of $\operatorname{IgG} 2 a, \lg G 2 b$ and $\lg G 3^{65}$. Among all the IgG subclasses, $\operatorname{IgG} 2 \mathrm{~b}$ and IgG2a are considered to be the most potent IgGs regarding the activation of effector responses, which is normally attributed to their higher capacity to fix complement.

IgGs perform their effector functions through different types of activating and inhibitory FcyRs ${ }^{66}$. FcyRs can be divided in two groups according to their ability to bind the Fc domain of IgGs. Type I FcyRs recognize the Fc region in its open conformation, whereas type II FcyRs recognize the Fc region in its closed conformation. Both in humans and mice, different type I FcyRs activating receptors and one inhibitory receptor can be found. In mice, there are three activating FcyRs (FcyRI, FcyRIII and FcyRIV), which consist of a specific alpha-chain and a common $\gamma$-chain, which is associated to intracellular immunoreceptor tyrosine-based activation motifs (ITAMs). In contrast, the inhibitory receptor FcyRIllb consists of a single alpha chain associated to intracellular immunoreceptor tyrosine-based inhibition motifs (ITIMs) ${ }^{67}$. These receptors are expressed by macrophages, neutrophils, eosinophils, dendritic cells and NK cells among other cell types. 


\subsubsection{IgE}

The structure of IgE resembles that of IgGs, but IgE contains one further constant domain in its heavy chain at the Fc fragment. IgE has a molecular weight of $190 \mathrm{kDa}$ and is the least abundant Ig isotype in plasma, having in mice a normal serum concentration of 0-0001-0.001 mg/ml $\mathrm{m}^{62}$. The role of $\mathrm{IgE}$ in immune responses against parasites and within allergic reactions has been extensively documented ${ }^{68}$. For example, worm infection induces a strong Th2 immune response associated with mastocytosis, eosinophilia, antibody class-switching to produce IgE and production of IL-4, IL-5 and IL-13 ${ }^{69}$. In allergic reactions, mast cells and basophils release their cytoplasmic granules upon cross-linking of the cognate allergen with IgE molecules, which are constitutively located at the membrane of these cells ${ }^{70}$. These granules trigger local inflammation, as they contain several inflammatory molecules such as histamine, serine proteases, carboxypeptidase A and proteoglycans.

The Fc receptors that recognize the Fc domain of IgE constitute the Fce receptor family, and include a high affinity and a low affinity receptor, which do not share any structural similarity ${ }^{71,72}$. The high affinity receptor, $\mathrm{Fc \varepsilon RI}$ is a heterotetramer composed by one $\alpha$ subunit, one $\beta$ subunit and a homodimer of the $\mathrm{Fc} \gamma \mathrm{R}$ common $\gamma$-chain. The FcyR common $\gamma$-chain homodimer, together with the $\beta$ subunit, promote the downstream signalling cascade following engagement of IgE. Mast cells and basophils express FcERI in their membrane, which upon crosslinking with IgE mediates degranulation and cytokine release. The low affinity receptor for IgE, FceRII or CD23, belongs to the C-type lectins receptors. FceRII is formed by trimers that self-associate through their extracellular domains forming an alpha-helical coiled-coil structure, where the lectin domains hold together at the end of the whole structure. B cells express FceRII together with the FcyRIIB, with both receptors being implied in the regulation of IgE production.

\subsection{5. $\lg A$}

IgA is the second most prevalent antibody found in human serum after IgG, having a normal concentration of 2-3 $\mathrm{mg} / \mathrm{ml}$. The Fc region of IgA monomers consists of three constant domains, and has a molecular weight of $160 \mathrm{kDa}$. The half-life of IgA in serum is short, and it is able to fix complement ${ }^{62,73,74}$. In humans, two isoforms of $\lg A$ exist ( $A 1$ and $A 2)$, but in mice just one isoform of IgA exists. This immunoglobulin can be found as a monomer or as a dimer when secreted, where a J chain together with disulfide bridges stabilize the dimeric structure. IgA production exceeds the production of other antibody isotypes when measured on a daily basis, and it plays a key role in immune protection by blocking pathogens from attaching to intestinal epithelial cells. This blocking process, known as immune exclusion, is driven by the direct recognition of receptor-binding domains from microbial pathogens or toxins, together with a series of events involving agglutination, entrapment in mucus and clearance through peristalsis ${ }^{75}$. 
Development of intestinal IgA is highly dependent on commensal colonization, since germ-free mice have reduced numbers of IgA+ ASC, and it has been shown to facilitate the uptake of antigen across the intestinal epithelium, facilitating antigen presentation and priming of adaptive immunity ${ }^{76}$. Although a Fc receptor for IgA exists in humans (the FcaRI which mediates IgA effects ${ }^{77}$ ), this is not the case in mice ${ }^{75}$. In these animals, IgA has been proposed to exert their mechanisms through the plgR, since KO animals for this receptor show 40 times more IgA serum levels than WT animals. It has also been reported to bind to the Fca/ $\mu \mathrm{R}$, presumably participating in antigen presentation ${ }^{52,78}$.

\subsection{Autoantibodies exert several effector mechanisms within the CNS}

Although the antigen triggering the immune response in MS has not been identified ${ }^{79,80}$, presence of oligoclonal bands in the CSF as well as therapeutic effects of plasmapheresis indicate a pathogenic role for autoantibodies in $\mathrm{MS}^{25-28}$. However, a directly pathogenic antibody specificity has not been identified in MS patients. Autoantibodies targeting neurofascin, contactin 2 and even the potassium channels KIR4.1 on astrocytes have been reported ${ }^{81}$. MOG is found on the surface of myelinating oligodendrocytes and external layers of myelin sheaths in the CNS, as well as MBP and PLP, being a potential target for autoantibodies ${ }^{82}$. Actually, MOG-specific antibodies were detected in children with MS and acute disseminated encephalomyelitis (ADEM) ${ }^{81}$. In total, it is estimated that $30 \%$ of patients with MS produce IgGs that have pathogenic potential| ${ }^{83}$.

In active CNS lesions of MS patients, IgG deposition and complement can be observed along with T cells and other infiltrating cells at sites of myelin breakdown ${ }^{30,31}$. In the EAE model, MOG autoantibodies have been shown to have high demyelinating capacity, which is dependent on complement fixation. However, MOG autoantibodies alone cannot trigger CNS inflammation, since they are not able to induce disease in naïve animals $^{84,38}$. In addition, administration of MOG autoantibodies at high doses at the EAE onset cause severe demyelination and exacerbation of the disease ${ }^{85,86,87}$.

Autoantibodies can contribute to disease pathogenesis within the CNS through different mechanisms. CNS demyelination can be enhanced by antibody-dependent cell cytotoxicity (ADCC) and activation of the complement cascade ${ }^{88,84}$. These two mechanisms are mediated by the antibodies' Fc part. In ADCC, the Fc region is recognized by immune cells expressing Fc receptors, leading to an inflammatory response in order to eliminate the target ${ }^{85}$. On the other hand, the complement binding region is found in the antibody Fc region ${ }^{89}$. Fixation of complement units in the Fc region triggers complement-dependent cell-mediated cytotoxicity (CDCC), where immune cells expressing complement receptors recognize the Fc region and attack the $\operatorname{target}^{85}$. One further potential demyelinating mechanism would be the activation of the complement cascade triggering the formation of the membrane attack complex (MAC) in the target cell membrane, a process known 
as complement-dependent cytotoxicity (CDC). Finally, the ability of an antibody to trigger these mechanisms is highly dependent on the isotype, since they have different affinities for Fc receptor and complement fixation ${ }^{66,84,90}$.

\subsection{CRISPR-Cas9 as a gene editing tool}

CRISPR (clustered regularly interspaced short palindromic repeats)-Cas (CRISPR associated protein) is kind of equivalent of an adaptive immune system in prokaryotes that uses RNA-guided nucleases to cleave foreign nucleic acids such as phages or plasmids ${ }^{91}$. In recent years, it has been widely used to target the genome of eukaryotic cells through codon-optimization of the Cas protein with an appropriate nuclear localization signal. The most widely used CRISPR-Cas system is the type II CRISPR-Cas9 system, which consist on a Cas9 nuclease coupled with a single guide RNA (sgRNA). The sgRNA is a chimeric RNA derived from the fusion of the crRNA and the tracrRNA (Figure 5). The Cas9 and sgRNA complex binds to the DNA target sequence that is complementary to the sgRNA, and to a protospacer adjacent motif (PAM) sequence, which is found directly after the target sequence and is required for target site recognition. Upon binding, the Cas9 makes double strand breaks (DSBs) in the genome and the cell will subsequently initiate DNA repair through NHEJ or homology-directed repair, depending on the availability of a DNA template. However, the CRISPR-Cas9 system has off-target problems since sequences sharing high homology with the sgRNA can be cleaved or mutated. Nevertheless, the CRISPR-Cas9 system allows genome editing through its simple design and high efficiency.

a Cas 9 programmed by crRNA:tracrRNA duplex

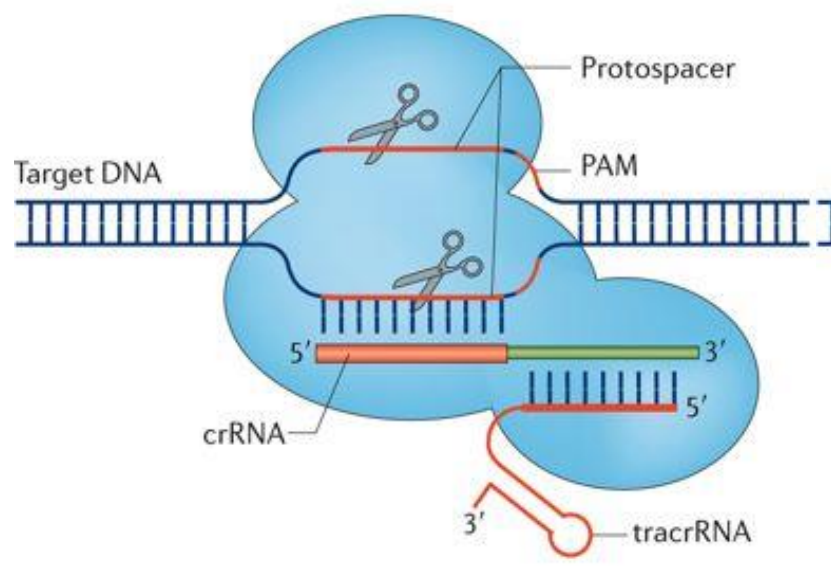

b Cas 9 programmed by single chimeric RNA

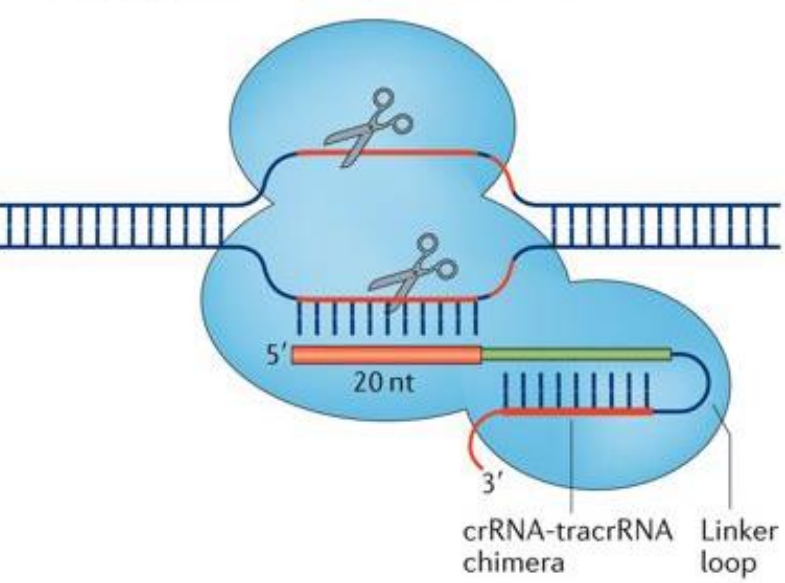

Figure 5. Cas9 requires gRNAs for cleaving the target DNA sequence. A) Cas 9 is guided to its target DNA by a crRNA and a tracrRNA or B) by a chimeric sgRNA resulting from the fusion, through a linker loop, of the crRNA and tracrRNA. Adapted from Gibson GJ et al. (2017) Nat Rev Rheu 


\section{Objectives}

B cells participate in the pathogenesis of multiple sclerosis and experimental autoimmune encephalomyelitis through different effector mechanisms. Recently, our department started to investigate the time frame and mechanisms by which MOG-specific B cells contribute to EAE development ${ }^{87}$. In this study, MOG-specific T cells and $B$ cells were tracked during the course of EAE using intravital two-photon imaging, functional characterization and genetic approaches. These experiments demonstrated that the presence of MOG-specific $B$ cells accelerated and enhanced the clinical course of EAE, but this effect was mediated by MOG-specific autoantibodies rather than other effector mechanisms of B cells. MOG-specific autoantibodies have been previously reported to aggravate EAE through demyelination ${ }^{92}$. However, in the setting used for our study, MOG-specific autoantibodies were applied at doses that did not exert additional myelin damage, but most likely acted through a different effector mechanism, which was relevant for the initial manifestation of the disease. Since both effects have been reported to be antibody-dependent, we hypothesized that the different effector mechanisms could be balanced when the accessibility of the antibodies (dosing) and their isotype were modified.

The general objective of this study was to evaluate the relevance of the isotype of the MOG-specific antibodies for the development of autoimmunity in the central nervous system using the EAE model. The first aim of this study was to generate different isotypes of MOG-specific antibodies produced by two hybridoma cell lines while preserving their specificity. These cell lines produce MOG-specific antibodies, but the epitope recognized by their antibodies is slightly different. To modify the antibody isotype, a CRISPR-Cas9 approach was designed. The generation of modified hybridoma cell lines producing antibodies of different isotypes included the characterization of immunoglobulins expressed at the membrane and secreted by the cells, targeting of the hybridoma cell lines and selection by cell sorting, establishment and characterization of a pure cell line producing exclusively the modified isotype, and large-scale production of purified antibody isotypes. The second aim of this study was to address the disease modifying effects of different MOG-specific antibody isotypes in the EAE model. First, MOG-specific antibodies of different isotypes were used in the active and transfer EAE models to evaluate their capacity to modulate the disease course and T cell reactivation within the CNS. Furthermore, since MOG-specific autoantibodies have been reported to exacerbate the disease through increased demyelination, the effects of different doses and different isotypes on demyelination as well as macrophage and T cell infiltration were investigated. Finally, since recognition of the antibody Fc part by immune cells expressing Fc receptors triggers antibody-dependent effector functions, we sought to address the role of Fc receptors in antibody effector mechanisms within the CNS using knock out animals for different Fc receptors, again applying MOG-specific antibodies of different isotypes. 


\section{Materials and Methods}

\subsection{Media and Buffers}

\section{RPMI medium}

Advanced RMPI 1640 Medium (Gibco ${ }^{\mathrm{TM}}$, ThermoFisher) was supplemented with 10\% Fetal Bovine Serum, 4 mM L-glutamine, $1 \%$ Gibco ${ }^{\circledR}$ Penicillin/Streptomycin (Penicillin (10000 U/ml) and 0,2\% $\beta$-mercaptoethanol (Roth, 99,9\%).

\section{Eagle's HEPES (EH) Medium}

133.8g DMEM powder (Gibco ${ }^{\text {TM }}$, ThermoFisher) was diluted in $10 \mathrm{~L}_{2} \mathrm{O}$, supplemented with $37 \mathrm{~g} \mathrm{NaHCO}_{3}$ and 25 mM HEPES (Life Technologies).

\section{T cell culture media (TCM)}

$1 \mathrm{LEH}$ medium was supplemented with $1 \% \mathrm{Gibco}^{\circledR}$ Penicillin/Streptomycin, $1 \% \mathrm{Gibco}^{\circledR}$ Non-essential amino acids (MEM NEAA, 100x, Life Technologies), 1\% of Sodium pyruvate (Gibco ${ }^{\circledR}$ ), 1\% L-asparagine (Sigma Aldrich), 1\% L-Glutamine (PAN-Biotech) and 10\% FCS.

\section{LB medium}

$25 \mathrm{~g}$ of $\mathrm{LB}$-medium (Luria/Miller, Roth ${ }^{\circledR}$ ) were diluted in $1 \mathrm{~L} \mathrm{H}_{2} \mathrm{O}$ and supplemented with $100 \mathrm{ng} / \mathrm{ml}$ of ampicillin.

\section{Phosphate Buffered Saline (1xPBS)}

$137 \mathrm{mM} \mathrm{NaCl}, 2.7 \mathrm{mM} \mathrm{KCl}, 10 \mathrm{mM} \mathrm{Na} 2 \mathrm{HPO} 4$ and $10 \mathrm{mM} \mathrm{NaH}_{2} \mathrm{PO}_{4}$ were added to $1 \mathrm{~L} \mathrm{H}_{2} \mathrm{O}$.

\section{FACs Buffer}

PBS was supplemented with 0,5\% bovine serum albumin (BSA, Roth) and $2 \mathrm{mM}$ Ethylenediaminetetraacetic acid tetrasodium salt hydrate (EDTA, Sigma Aldrich)

\section{Ammonium - chloride - cotassium (ACK) Lysis Buffer}

$0,15 \mathrm{M} \mathrm{NH}_{4} \mathrm{Cl}, 1 \mathrm{mM} \mathrm{KHCO}_{3}$ and 0,1 mM EDTA were diluted in $\mathrm{H}_{2} \mathrm{O}$ and the $\mathrm{pH}$ was adjusted to 7.4 with $\mathrm{NaOH}$ and $\mathrm{HCl}$.

\section{Gel electrophoresis buffer}


TRIS/Borate/EDTA buffer was prepared with 10,8 $\mathrm{g}$ TRIS, 5,5 g boric acid, and $4 \mathrm{ml} 0.5 \mathrm{M}$ EDTA, diluted in $1 \mathrm{~L}$ $\mathrm{H}_{2} \mathrm{O}$.

\section{Transfer buffer}

$25 \mathrm{mM}$ TRIS, 1,9 M glycin, 10\% SDS were diluted into $1 \mathrm{~L} \mathrm{H}_{2} \mathrm{O}$. The $\mathrm{pH}$ was adjusted to 8.3

\section{Agarose gels for cloning}

$0.6 \mathrm{~g}$ Agarose (Roth) in $60 \mathrm{ml} \mathrm{H} \mathrm{H}_{2}$ and heated up at $100^{\circ} \mathrm{C}$ for $3 \mathrm{~min}$ and $0.0001 \%$ ethidium bromide was added to the mix.

\section{PK Buffer for genomic DNA isolation}

$0.1 \mathrm{M}$ TRIS HCl and 0.05 EDTA were added to $0.5 \mathrm{ml} \mathrm{H} 2 \mathrm{O}$.

TBS-T

TBS was prepared by adding $24 \mathrm{~g}$ of TRIS and $88 \mathrm{~g}$ of $\mathrm{NaCl}$ to $1 \mathrm{~L} \mathrm{H}_{2} \mathrm{O}$, the $\mathrm{pH}$ was adjusted to 7.6 . TBS was supplemented with $0.05 \%$ tween 20 (Roth).

\section{PBS-T}

1X PBS was supplemented with $0.05 \%$ of Triton-X 100 (Roth).

TMB

TMB substrate reagent set $(\mathrm{BD})$ was mixed following manufacturer instructions.

\section{Ammonium- Chloride- Potassium (ACK) Lysis Buffer}

$0.15 \mathrm{M} \mathrm{NH} 4 \mathrm{Cl}, 10 \mathrm{mM} \mathrm{KHCO}, 0.1 \mathrm{mM}$ EDTA .2Na was diluted in $\mathrm{H} 2 \mathrm{O}$ and the $\mathrm{pH}$ was adjusted to 7.2-7.4 with $1 \mathrm{~N} \mathrm{HCl}$.

\subsection{Experimental procedures}

\section{Primer design for cloning of genomic DNA Ig sequences}

For the design of suitable primers for cloning and sequencing of the Igs $\mathrm{CH}$ regions, Balb/c reference sequences from were extracted from the National Center For Biotechnology (NCBI, USA). Several primers were designed to amplify the upstream region of the different isotypes, which contained restriction sites for subsequent cloning. The sequences of these primers are indicated in the table 1. 
Table 1. Designed primers for amplification of the sequence preceding the first IgH constant domain of each isotype is depicted.

\begin{tabular}{|l|l|}
\hline Upstream IghG1 forward & ggtcgacGGCAAAGACAGGAAGGGACTGTTAG \\
\hline Upstream IghG1 reverse & cggatccTCAGGGAAATAGCCCTTGACCAGGC \\
\hline Upstream IghE forward & gggatccATAATGGCTTCCAGTCTGCCACCCAG \\
\hline Upstream IghE reverse & cgaattcGGGTCATGGAAGCAGTGCCTTTACAGGGC \\
\hline Upstream IghG2b forward & gggatccCATGGATAGAGACCTAGAAGCAAGA \\
\hline Upstream IghG2b reverse & cgaattcTAGACTGATGGGGGTGTTGTTTTGG \\
\hline
\end{tabular}

\section{Genomic DNA extraction and Region of interest PCR amplification}

In order to extract crude genomic DNA (gDNA) from the 8.18c5 and Z2 hybridoma cell lines, a proteinase Kbased protocol was used. Briefly, 1 to 2 million cells were transferred to $1.5 \mathrm{ml}$ Eppendorf tubes. The cells were centrifuged for $6 \mathrm{~min}$, at $4^{\circ} \mathrm{C}, 1200 \mathrm{rpm} / 301 \mathrm{~g}$ and the supernatant was removed. The cell pellet was incubated overnight at $55^{\circ} \mathrm{C}$ with a solution containing $20 \mu \mathrm{l}$ of proteinase $\mathrm{K}(200 \mathrm{ng} / \mu \mathrm{l}), 10 \mu \mathrm{l}$ of $10 \% \mathrm{SDS}$ and $1 \mathrm{ml}$ of PK-Buffer. After incubation, the gDNA was precipitated with isopropanol and centrifuged for $10 \mathrm{~min}$, at $4^{\circ} \mathrm{C}, 4000 \mathrm{rpm} / 3345 \mathrm{~g}$. The isopropanol was removed and the gDNA was washed with 70\% ethanol, centrifuged ( $5 \mathrm{~min}$, at $4^{\circ} \mathrm{C}, 4000 \mathrm{rpm} / 3345 \mathrm{~g}$ ) and air-dried for $10 \mathrm{~min}$. The pellet was then diluted in $500 \mu \mathrm{l}$ of RNAse free water (Invitrogen), and DNA concentration was measured by Nanodrop.

Regular PCR amplification of the desired loci was performed in a total volume of $40 \mu \mathrm{l}$, consisting on: $2 \mu \mathrm{M}$ of forward primer; $2 \mu \mathrm{M}$ of reverse primer; $2 \mu \mathrm{M}$ of gDNA; $20 \mu$ lof CloneAmp (CloneTech) and RNAse free water up to a final volume of $20 \mu \mathrm{l}$. The PCR conditions for amplification of the upstream IgH regions of every isotype were: $94^{\circ} \mathrm{C}$ for $1 \mathrm{~min}$ denaturation, $98^{\circ} \mathrm{C}$ for 30 seconds denaturation, $60^{\circ} \mathrm{C}$ annealing temperature for 15 seconds, $72^{\circ} \mathrm{C}$ extension for 30 seconds. 20 cycles were performed for every DNA fragment.

\section{DNA extraction and validation}

Amplified DNA was visualized with UV light and extracted using a cutter. After that, pure DNA was extracted using GeneJet gel extraction kit (ThermoFisher) following the manufacturer instructions. DNA cut with restriction enzymes was used to validate the amplified DNA sequence. 


\section{Bacteria and medium}

Stellar ${ }^{\mathrm{TM}}$ competent cells from TAKARA were used for transformation (30 $\mu$ //vector) using SOC medium contained within the kit. SOC medium was also prepared following the composition: $2 \%$ tryptone, $0.5 \%$ yeast extract, $10 \mathrm{mM} \mathrm{NaCl}, 2.5 \mathrm{mM} \mathrm{KCl}, 10 \mathrm{mM} \mathrm{MgCl}_{2}, 10 \mathrm{mM} \mathrm{MgSO}_{4}$, and $20 \mathrm{mM}$ glucose.

\section{Agar plates}

$10 \mathrm{~g}$ Tryptone, $5 \mathrm{~g}$ yeast extract, $5 \mathrm{~g} \mathrm{NaCl}$ and $15 \mathrm{~g}$ Agar were diluted in $1 \mathrm{~L} \mathrm{H}_{2} \mathrm{O}$. The mix was autoclaved (Systec VX-150). After, the mix was heated up until $55^{\circ} \mathrm{C}$ and ampicillin was added $(25 \mathrm{ng} / \mathrm{ml})$.

\section{Cloning into reference vector and sequencing}

Purified DNA was cloned into the cloning vector pJET1.2/blut contained in the CloneJET PCR cloning Kit (Thermo Scientific) following manufacturer instructions. Blunted DNA was transformed into Stellar bacteria (Takara). Briefly, $30 \mu$ l of competent bacteria were incubated with the DNA for 20 minutes at $4^{\circ} \mathrm{C}$, followed by a heat shock at $42^{\circ} \mathrm{C}$ for 45 seconds. After that, $1 \mathrm{ml}$ of SOC media was added to the mix, and incubated for 1 hour at $37^{\circ} \mathrm{C}$ while shaking. The cell suspension was plated in agar plates containing ampicillin and incubated overnight at $37^{\circ} \mathrm{C}$. The resulting DNA vector was purified with GeneJet Plasmid Miniprep Kit (Thermo Scientific) following the manufacturer instructions. Insert orientation was tested with restriction enzymes. Forward and reverse PJET primers (Thermo Scientific) were used for sequencing. Sanger sequencing results were compared to the NCBI reference sequence in order to detect polymorphisms. After confirmation of the sequence, several guide-RNAs were designed for cloning into DNA vectors containing the sequence for the Cas9 protein (Table 4).

\section{Cloning of protospacers into Cas-9-expressing vectors}

$10 \mu \mathrm{M}$ Forward and reverse oligonucleotides were mixed together, and annealing and phosphorylation was performed according to the following protocol: denaturation at $95^{\circ} \mathrm{C}$ for $5 \mathrm{~min}, 25^{\circ} \mathrm{C}$ for $1 \mathrm{~min}$, ramping program $\left(0.1^{\circ} \mathrm{C} / \mathrm{s}\right)$ until $12^{\circ} \mathrm{C}$. Cloning of these oligonucleotides into the Cas9-expressing vectors was performed following Zhang's Lab protocols ${ }^{91}$.

\section{Cherry recombinant cleavage assay}

The sequences used for protospacers design were extracted from pJET vectors, and introduced by enzymerestricted based cloning into CAG-Cherry vectors. This vector contains restriction sites between two homology arms that were used for cloning of the target sequence. Further information about this assay is provided in the chapter 4.1.3. and Figure 9. $1 \times 10^{5}$ HEK 293T cells were transfected with $10 \mu \mathrm{g}$ of CAG-Cherry vectors and $10 \mu \mathrm{g}$ of Cas-9 vectors cloned with the protospacers for the target sequence, or empty Cas-9 vectors. The DNA 
constructs were introduced into the HEK 293T cells using Lipofectamine 2000 (Thermo Scientific), following the manufacturer instructions. 48 hours the cells were evaluated by fluorescence microscopy with a Zeiss microscope, and the mean fluorescence intensity (MFI) was measured by FACs (FACs Calibur).

\section{Introduction of DNA constructs into $8.18 \mathrm{c5}$ and $Z 2$ hybridoma cell lines}

$10 \mu \mathrm{g}$ of DNA were introduced into $1 \times 10^{5} 8.18 \mathrm{c} 5$ or Z2 hybridoma cells by electroporation (Bio-Rad GenePulser Xcell ${ }^{\mathrm{TM}}$ ). The cells were diluted in $800 \mu \mathrm{l}$ of sterile PBS and received a pulse of $625 \mathrm{~V}$ following an exponential protocol (Impedance $50 \mu \mathrm{F}$, resistance infinite and $4 \mathrm{~mm}$ cuvette size). Electroporated cells were then washed with sterile PBS and transferred to 6 well-plates containing RPMI medium.

\section{Hybridoma culture and production of monoclonal alpha MOG antibodies}

The monoclonal $\alpha-M O G$ antibodies were derived from the $8.18 c 5$ and $Z 2$ hybridomas cell lines. The cell lines were expanded in RPMI medium until the cell density reached a concentration of $1400 \mathrm{cells} / \mathrm{cm}^{2}$ or 2.528 cells $/ \mathrm{cm}^{2}$. Once the appropriate cell density was reached, the culture medium was exchanged to PFHM-II (Thermo Fisher) and cells were kept in culture for 14 days. After this time, cell culture supernatants were harvested, centrifuged at $880 \mathrm{rpm} / 162 \mathrm{~g}$ for 8 minutes and filtered through $0.2 \mu \mathrm{m}$ filters (Sarsted, INC.). Protein purification of $\lg G$ antibody isotypes (IgG1, IgG2a and $\lg G 2 \mathrm{~b}$ ) was carried out using $1 \mathrm{ml}$ HiTrap Protein G High Performance Columns (GE Healthcare) following the manufacturer instructions. IgE and IgA antibody isotypes were purified using $1 \mathrm{ml} \mathrm{HiTrap} \mathrm{Protein} \mathrm{L} \mathrm{High} \mathrm{Performance} \mathrm{Columns} \mathrm{(GE} \mathrm{Healthcare)} \mathrm{following} \mathrm{the}$ manufacturer instructions. The eluted fractions were desalted using PD-10 Desalting columns (GE Healthcare) following the manufacturer instructions. The proteins were eluted in PBS and quantified using Nanodrop (Peqlab). The IgG1 (Ultra Leaf MOPC-21, BioXCell), IgG2a (Ultra Leaf MOPC-173), IgG2b (Ultra Leaf 27-35), IgE ( Ultra Leaf MEA-36) isotype control were purchased.

\section{Sodium dodecyl sulfate polyacrylamide gel electrophoresis (SDS-PAGE)}

SDS-PAGE ${ }^{93}$ assays were performed using two different polyacrylamide gels at two different Polyacrylamide concentrations. For the running gel, 1,33 ml 30\% Acrylamide mix were diluted into 2,42 $\mathrm{ml} \mathrm{H2O}$. 1,22 $\mathrm{ml}$ of 1,5M Tris-SDS buffer ( $\mathrm{pH} 8,8)$ was added to the mix, vortexed, and polymerization was achieved by adding 50 $\mu \mathrm{l}$ of ammonium persulfate (APS) and $20 \mu \mathrm{l}$ of TEMED. For the stacking gel, 0,22 $\mathrm{ml} 30 \%$ Acrylamide mix were diluted into $1.02 \mathrm{ml}$ of $\mathrm{H}_{2} \mathrm{O} .0 .44 \mathrm{ml} 1.5 \mathrm{M}$ Tris-SDS buffer ( $\mathrm{pH} 6.6$ ) were then added to the mix, plus $50 \mu \mathrm{l}$ of APS and $20 \mu$ of TEMED. 


\section{Gel electrophoresis and Western Blot}

Molecular weight and isotype of the purified monoclonal antibodies was confirmed by gel electrophoresis. Briefly, $1 \mu \mathrm{g}$ of protein was denatured in a $\beta$-mercaptoethanol-containing buffer. The denatured protein was then loaded into a SDS-PAGE, together with a molecular weight marker (PAGE ruler Prestained plus, Thermo scientific). The SDS-PAGE was then transferred into a BIO-RAD electrophoresis cassette and run for 90 min at $120 \mathrm{~V}$.

For the molecular weight characterization, the SDS-PAGE gels were stained with Cromassie blue for 5 min and washed overnight with water. For the Western Blot ${ }^{94}$ characterization of the isotype, the SDS-PAGE was transferred to a transfer cassette together with a nitrocellulose membrane, and over- and under-laid with filter paper and sponges. Protein transfer to the nitrocellulose membrane was performed at $100 \mathrm{~V}$ for 1 hour at $4^{\circ} \mathrm{C}$. After that, the nitrocellulose membrane was washed with $\mathrm{H} 2 \mathrm{O}$, and blocked for 90 min with TBST-BSA $5 \%$. Following blocking the membrane was transferred to a TBST and BSA (TBST-BSA 5\%) solution containing the primary antibody (biotin anti-Ig, 1:40000 dilution), and incubated overnight at $4^{\circ} \mathrm{C}$. The next day the membrane was washed with TBST at room temperature, and transferred to a TBST-BSA 5\% solution containing the secondary antibody (Streptatividin-APC, 1:8000 dilution), and incubated for 2 hours at room temperature. Both Cromassie blue stained gels and immunostained nitrocellulose membranes were then scanned in a GE Amershamer Imager 600 scanner (GE Healthcare).

\section{Hybridoma supernatant ELISA}

Determination of the antibody isotype was achieved by ELISA. In brief, ELISA plates were coated with $4 \mu \mathrm{g} / \mathrm{ml}$ recombinant MOG (rMOG) in PBS and incubated overnight at $4^{\circ} \mathrm{C}$. Plates were washed with PBS plus $0.05 \%$ of Tween20 (Roth) (PBS-T), and blocked with PBS plus 5\% BSA for 1 hour. After blocking, the plates were washed and incubated for two hours with hybridoma culture supernatants at room temperature. Following probe incubation, the plates were washed, and incubated for $90 \mathrm{~min}$ with biotin-conjugated anti-lg antibodies in a concentration of $1 \mu \mathrm{g} / \mathrm{ml}$, and ST-HRP antibodies in a $2 \mu \mathrm{g} / \mathrm{ml}$. Finally, after a further washing step, HRP enzymatic reaction with its substrate 3,3', 5,5'tetramethyilbenzidine (TMB) was induced by incubation for 30 min of $100 \mu \mathrm{l}$ of TMB per well, and stopped by addition of $50 \mu \mathrm{l} 1 \mathrm{M} \mathrm{H}_{2} \mathrm{SO}_{4}$. Absorbance was then measured at $450 \mathrm{~nm}$ with correction wavelength of $620 \mathrm{~nm}$ in a Sunrise ${ }^{\mathrm{TM}}$ ELISA plate reader (Tecan Life Sciencies).

\section{Flow cytometry}

Isolated cells from tissues were stained with monoclonal antibodies conjugated with fluorochromes. Hybridoma cells were stained with both monoclonal antibodies conjugated with fluorochromes, and with biotin-conjugated anti-Ig antibodies plus ST-APC antibodies. Cells were stained in $100 \mu$ I FACs buffer for 15 min at $4^{\circ} \mathrm{C}$. This process was repeated for second antibody staining. After that the cells were washed with $2 \mathrm{ml}$ 
FACs buffer and resuspended in EH+EDTA or RPMI medium. A further staining step with ST-APC antibodies was performed when hybridoma cells were stained.

For the assays with T-mMOG cells for testing specificity and isotype of the new purified antibodies derived from $8.18 \mathrm{c} 5$ and $Z 2$ this protocol was followed, but with a previous incubating the $8.18 \mathrm{c} 5$ and $\mathrm{Z} 2$ purified antibodies for $15 \min$ at $4^{\circ} \mathrm{C}$.

For cell sorting, The BD FACs Aria II Cell Sorter was used and the sorted cells were collected in EH+10\% FCS. For quantification, $100 \mu \mathrm{l}$ of PBS containing fluorescent beads were added to the cell suspension at the same volume. Quantification measurement were repeated three times using the BD FACs Calibur Cell Analyzer. Results obtained with both protocols were analysed with FlowJo software (Tree Star, Inc., USA). The antibodies used in this work are specified in Table 2:

Table 2. All antibodies used for FACs staining are depicted.

\begin{tabular}{|c|c|c|c|}
\hline Specificity & Clone & Label & Company \\
\hline CD45.2 & 104 & PE & BioLegend \\
\hline CD4 & H129.19 & PE-Cy5 & BioLegend \\
\hline CD3 & $17 A 2$ & APC & BioLegend \\
\hline IgG1, Isotype ctrl & RTK2071 & PE & BioLegend \\
\hline PE-IgG1 & RMG1-1 & PE & BioLegend \\
\hline Biotin-IgG1 & RMG1-1 & Biotin & BioLegend \\
\hline Biotin-IgG2b & RMG2b-1 & Biotin & BioLegend \\
\hline Biotin-IgG2a & RMG2a-62 & Biotin & BioLegend \\
\hline Biotin-IgE & RME-1 & Biotin & BioLegend \\
\hline Biotin-IgA & RMA-1 & Biotin & APC \\
\hline Streptavidin APC & $X$ & & \\
\hline
\end{tabular}

\section{RNA isolation, cDNA synthesis and quantitative real-time PCR (qRT-PCR)}

Sorted cells were spun down for $6 \mathrm{~min}$ at $4^{\circ} \mathrm{C}, 4000 \mathrm{rpm} / 3345 \mathrm{~g}$ in a Centrifuge $5415 \mathrm{R}$ (Eppendorf). The supernatant was discarded and the cells were resuspended in $350 \mu$ l of Qlazol ${ }^{\circledR}$ Lysis Reagent (Qiagen Sciences). $70 \mu \mathrm{l}$ of chloroform were added to the suspension, and after 20 min incubation at room temperature, the samples were centrifuged for $15 \mathrm{~min}$ at $4^{\circ} \mathrm{C}, 12000 \mathrm{~g}$. The aqueous phase was then transferred to a different tube and RNA precipitation was achieved by addition of $175 \mu$ of isopropanol. $1 \mu$ l of glycogen was added to the samples and they were incubated overnight at $-20^{\circ} \mathrm{C}$. The next day, the samples were incubated for 10 min at room temperature, and centrifuged at $12000 \mathrm{~g}$ for $10 \mathrm{~min}$ at $4^{\circ} \mathrm{C}$. Supernatant was discarded, the RNA 
pellet was washed with $350 \mu \mathrm{l}$ of $75 \%$ ethanol and the samples were centrifuged for $10 \mathrm{~min}$, at $4^{\circ} \mathrm{C}, 12000 \mathrm{~g}$. Finally, the pellet was air dried for 10 to $15 \mathrm{~min}$ at room temperature, and resuspended in $12 \mu$ of Ultrapure ${ }^{\mathrm{TM}}$ Distilled DNAse/RNAse free Water (Invitrogen).

The RevertAid First Strand cDNA Synthesis kit (Thermo Scientific) was used for retroconversion to cDNA following the manufacter's protocol. The qRT-PCR StepOnePlus ${ }^{\mathrm{TM}}$ Real-Time PCR System (Applied Biosystems) was used for the qRT-PCR assays. The cDNA-converted samples were then mixed with a qPCR Master Mix (Eurogentec) and target-specific TaqMan ${ }^{\circledR}$ probes labelled with FAM $^{\mathrm{TM}}$ (6-carboxyfluorescein) and quenched by TAMRA ${ }^{\mathrm{TM}}$ (6-carboxytetramethylrhodamine). Results were analysed with the StepOne-Plus software v2.0. Expression of IFNY, IL-17 and GM-CSF was normalized to HPRT. qPCR primers are listed in Table 3.

Table 3. List of all primers used for qRT-PCR.

\begin{tabular}{|c|c|}
\hline HPRT & $\begin{array}{l}\text { Fw (5`-3`) TGCTCGAGATGTCATGAAGG } \\
\text { Rev (5`-3’) TATGTCCCCCGTTGACTGAT } \\
\text { Probe [6FAM]ATCACATTGTGGCCCTCTGT[TAM] }\end{array}$ \\
\hline IFNY & $\begin{array}{l}\text { Fw (5’3`) TCAAGTGGCATAGATGTGGAAGAA } \\
\text { Rev (5`-3’) TGGCTCTGCAGGATTTTCATG } \\
\text { Probe [6FAM]TCACCATCCTTTTGCCAGTTCCTCCAG [TAM] }\end{array}$ \\
\hline IL-17A & $\begin{array}{l}\text { Fw (5`-3`) ACTTTCAGGGTCGAGAAGATGCT } \\
\text { Rev (5`-3`) TTCTGAATCTGCCTCTGAATCCAC } \\
\text { Probe [6FAM]TGGGTGTGGGCTGCACCTGC [TAM] }\end{array}$ \\
\hline GM-CSF & $\begin{array}{l}\text { Fw(5’-3’) GGGCGCCTTGAACATGAC } \\
\operatorname{Rev}\left(5^{\prime} 3^{\prime} 3^{\prime}\right) \text { CGCATAGGTGGTAACTTGTGTTTC } \\
\text { Probe [6FAM]CCCCCCAACTCCGGAAACGGA [TAM] }\end{array}$ \\
\hline
\end{tabular}

\section{Animals}

All animal experiments were approved by the Lower Saxony state authority LAVES (Niedersächsisches Landesamt für Verbraucherschutz und Lebensmittelsicherheit). Mice breeding was carried out in the University Medical Center Göttingen (UMG) under specific pathogen-free conditions. For the in vivo studies, different mouse lines (all on the $\mathrm{C57BI} / 6 \mathrm{~J}$ genetic background) were used: Wildtype, $\mathrm{F} c \gamma \mathrm{R}+\mathrm{IIB} \mathrm{KO}{ }^{95}$ (crossing

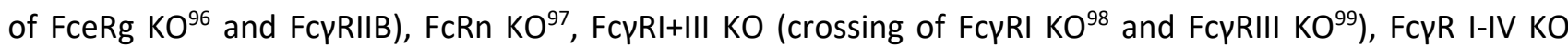

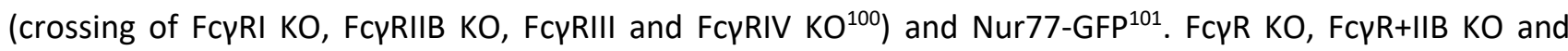
FcpRI+III KO animals were kindly provided by F. Nimmerjahn, University of Erlangen-Nürnberg, Germany. FcpR I-IV KO animals were kindly provided by J.S. Verbeek, Leiden University Medical center, The Netherlands. 


\section{EAE Direct immunization}

10 to 12 weeks old mice were intraperitoneally anesthetized using ketamine ( $80 \mathrm{mg} / \mathrm{kg}$, Medistar) plus xylazine ( $8 \mathrm{mg} / \mathrm{kg}$, Riemser Arzneimittel) in 0,9\% NaCl (Braun). Mice were subcutaneously immunized at the tail basis with $25 \mu \mathrm{g}$ (suboptimal immunization) or $50 \mu \mathrm{g}$ (optimal immunization) of MOG $_{35-55}$ peptide (MEVGWYRSPFSRVVHLYRNGK, purchased from Charité, Institute for Medical Immunology, Berlin) in PBS, emulsified in an equal volume of Complete Freund's adjuvant (CFA) containing Mycobacterium tuberculosis H37RA (Difco) at a final concentration of $1 \mathrm{mg} / \mathrm{ml}$.

Mice were treated with 2 consecutive i.p. injections of Pertussis Toxin (PTX, List Biological Laboratories; 400 $\mathrm{ng} /$ mouse in total), one immediately after immunization and 2 days post immunization.

\section{Transfer EAE}

Mice were immunized following the previously specified protocol with $75 \mu \mathrm{g} \mathrm{MOG}$ 35-55 peptide per animal. Shortly before disease onset (days 10 to 12 post immunization), animals were sacrificed by $\mathrm{CO}_{2}$ inhalation and draining lymph nodes were extracted. Lymph nodes were then pressed through $70 \mu \mathrm{m}$ metal strainers to obtain single cell suspensions. After quantifying the number of cells, the cell suspensions were diluted in mouse $\mathrm{T}$ cell culture medium to a final concentration of $5^{*} 10^{6}$ cells $/ \mathrm{ml}$ and supplemented with $25 \mu \mathrm{g} / \mathrm{ml}$ $\mathrm{MOG}_{35-55}, 25 \mathrm{ng} / \mathrm{ml}$ recombinant mouse IL-12 (R\&D) and $20 \mu \mathrm{g} / \mathrm{ml}$ of $\alpha$-IFN Ab (BioXcell, clone XMG1.2) for re-stimulation. Cell suspensions were plated in $96-$ well round bottom plates (100 $\mu \mathrm{l} /$ well, Nunclon $^{\mathrm{TM}}$ Delta Surface, Thermoscientific) and cultured at $37{ }^{\circ} \mathrm{C}, 5 \% \mathrm{CO}_{2} .2$ days later, the cells were transferred to 96 -well flat bottom plates (Nunclon ${ }^{\mathrm{TM}}$ Delta Surface, Thermoscientific) and 24 hours later were harvested. After washing, the cell suspensions were diluted in $\mathrm{EH}$ medium to a final concentration of $2.5 \times 10^{6}$ cells and i.p. injected into recipient animals.

\section{EAE assessment}

Animals were daily examined from $d 8$ post immunization or on $d 2$ post T-cell transfer until the end of the experiment for clinical signs of disease on a scale from 0 to 10 depending on severity. Clinical scores were assigned according to the following criteria: 0 , healthy; 1 , reduced tone of tail; 2 , limp tail, impaired righting; 3, absent righting; 4, gait ataxia; 5, mild paraparesis of hindlimbs; 6, moderate paraparesis; 7, severe paraparesis; 8, tetraparesis; 9, moribund; 10, death. Animals achieving score 7 or higher were sacrificed due to ethical concerns. 


\section{Isolation of immune cells from the CNS}

Mice were sacrificed by $\mathrm{CO}_{2}$ inhalation and spinal cord was extracted. Spinal cord parenchyma and meninges were separated and kept in cold EH medium plus 2 mM EDTA (EH+EDTA). Tissues were subsequently pressed through a $70 \mu \mathrm{M}$ metal strainer in order to obtain a single cell suspension. 90\% PercollTM (GE Healthcare) was added to the cell suspension, mixed by inversion of the tube and centrifuged $\left(30 \mathrm{~min}, 2780 \mathrm{rpm} / 1615 \mathrm{~g}, 4^{\circ} \mathrm{C}\right.$, w/o acceleration or break, Multifuge 1S-R, Heraeus). Finally, the cells were resuspended in FACs Buffer and filtered through $40 \mu \mathrm{m}$ filters before staining.

\section{Isolation of lymphocytes from the blood}

Mice were sacrificed by $\mathrm{CO}_{2}$ inhalation and blood was extracted by heart puncture using $100 \mu$ l $80 \mathrm{mM}$ EDTAcoated syringes. Blood samples were then diluted to a final volume of $1 \mathrm{ml}$ with PBS and carefully pipetted on top of Lymphocyte separation medium (LSM 1077, GE Healthcare). After centrifugation (30 min, 2000 rpm / $836 \mathrm{~g}, \mathrm{RT}, \mathrm{w} / \mathrm{o}$ acceleration or break, Multifuge 1S-R, Heraeus) the interphase was collected and washed with cold PBS. Erythrocytes lysis was performed by incubation of the cell suspension with ACK lysis buffer for $1 \mathrm{~min}$ on ice, and after that cells were spun down (1200 rpm / $301 \mathrm{~g}, 6 \mathrm{~min} 4^{\circ} \mathrm{C}$, Multifuge $1 \mathrm{~S}-\mathrm{R}$, Heraeus) and resuspended in FACs Buffer.

\section{Histology}

For the histology experiments, animals were transcardially perfused at the specified time points with PBS for 10 min at $4^{\circ} \mathrm{C}$, and 10 min with $4 \%$ PFA at $4^{\circ} \mathrm{C}$. Spinal cord samples were kept in PFA for 5 days, and after that they were dehydrated and included in paraffin. $3 \mu \mathrm{m}$ thick sections were cut with a LEICA SR 2000R microtome and visualized in a Zeiss Axioskop 40 microscope.

\section{Luxol Fast Blue}

Luxol fast blue (LFB) staining was performed according to the original protocol with some modifications ${ }^{102}$. In brief, after dehydration, the samples were incubated at $56^{\circ} \mathrm{C}$ in a $0,1 \% \mathrm{LFB}$ solution. After that, the slides were washed with $96 \%$ ethanol and $\mathrm{H}_{2} \mathrm{O}$. Excess of LFB was removed with $0.1 \% \mathrm{Li}_{2} \mathrm{CO}_{3}$ for 30 s, followed by washing in $70 \%$ ethanol and $\mathrm{H}_{2} \mathrm{O}$. After that, the slides were incubated during $10 \mathrm{~min}$ in $0.8 \%$ Periodic Acid, washed in $\mathrm{H}_{2} \mathrm{O}$, and further incubated in Schiff's reagent for 20 additional minutes. The excess of which was washed away with a sulphite wash. Finally, the cuts were washed $10 \mathrm{~min}$ in tap water, dehydrated and mounted. Analysis of percentage of demyelination was performed in ImageJ v1.8. Spinal cord white matter area was calculated by subtracting the grey matter area to the total area. Summation of the demyelinating lesions was then used for calculation of percentage of demyelination. 


\section{Immunohistochemistry}

For antigen retrieval, the slides were then boiled in citrate buffer for $15 \mathrm{~min}$, washed with PBS, and endogenous peroxidase activity was blocked with $3 \% \mathrm{H}_{2} \mathrm{O}_{2}$ for $15 \mathrm{~min}$ at $4^{\circ} \mathrm{C}$. Biotinylated anti-mouse MAC3 and CD3 antibodies (1:200, BD biosciences), which were incubated overnight at $4^{\circ} \mathrm{C}$. Secondary streptavidinHRP antibodies (1:200, DB biosciences) were incubated for 4 hours at room temperature. the peroxidasebased $A B C$ detection system (DakoCytomation) in combination with diaminobenzidine was used for visualization, and all sections were counterstained with hematoxylin. Quantification of MAC-3 stainings was performed in image $v 1.8$. In brief, total spinal cord area was calculated and colour deconvolution was applied to the image (pre-set: H DAB). The brown channel was selected, light background was removed and threshold was established based on the staining intensity. Area occupied by the staining was then related to the total area, obtaining the percentage of area occupied by the staining. CD3+ T cells were quantified in image v1.8 and number of cells per $\mathrm{mm}^{2}$ was calculated considering the total area of the section.

\section{Statistics}

Animals were randomly assigned to the different $8.18 c 5, \mathrm{Z2}$ or isotype control groups. Statistical analysis was performed using GraphPad Prism 8. T test was used for analysis of EAE day of onset. One-way Anova plus Sidak's multiple comparison test was done for the analysis of the Nur-77 GFP experiments, for histological analysis (LFB, MAC3 and CD3) and for cytokine expression of ex vivo sorted T cells.

All data is depicted as Mean+/-SEM (standard error of the mean). P-values of statistical differences correspond to:

$$
\begin{aligned}
& P \geq 0,05 \text { ns } \\
& P=0,01 \text { to } 0,05^{*} \\
& P=0,001 \text { to } 0,01 * * \\
& P=0,0001 \text { to } 0,001 * * * \\
& P<0,0001 * * * *
\end{aligned}
$$

Statistical test used for the analysis are summarized here and are not displayed in the Figure legends. 


\section{Results}

\subsection{CRISPR-Cas9-driven isotype switch enables the generation of MOG-specific antibodies with different isotypes while preserving antigen specificity}

\subsection{8c5 hybridoma cells produce exclusively MOG-specific IgG1 antibodies}

Recent studies have pointed to a disease-modulatory role of MOG-specific antibodies in the CNS draining lymph nodes, where these autoantibodies triggered activation and expansion of MOG-specific T cells in an Fcdependent manner ${ }^{86}$. In a complementary and parallel study, previous results from our department indicated that MOG-specific antibodies exert disease-modifying effects in EAE such as acceleration of disease onset and a more severe clinical course of the disease, within the CNS rather than in the periphery ${ }^{87}$. Both studies made use of murine monoclonal MOG-specific antibodies derived from the 8.18c5 hybridoma, which produce IgG1 antibodies, using the $\gamma 1$ heavy chain locus. ${ }^{84}$. Upon transcription, two different IgG1 mRNA transcripts can be generated in an alternative splicing-dependent manner (Figure 6A). mRNA transcripts containing the M1 and M2 exons will be translated and transported to the cell membrane, being part of the BCR complex, and enabling its detection by FACS. Fluorescent staining for $\lg G 1$, and all isotypes localized downstream of $\lg G 1$, revealed that the $8.18 \mathrm{c} 5$ hybridoma exclusively express IgG1 antibodies at the membrane level (Figure 6B). Alternative splicing-driven excision of the $\mathrm{M} 1$ and $\mathrm{M} 2$ exons generates mRNA transcripts that, after translation in the endoplasmic reticulum, are packed in vesicles and secreted to the extracellular media. To further substantiate that the antibodies secreted by the 8.18c5 hybridoma are exclusively of the IgG1 isotype, we established a thymoma cell line expressing tomato-labelled mouse MOG at the cell surface (T-mMOG). FACS staining of T-mMOG cells with affinity chromatography-purified antibodies from 8.18c5 hybridoma cells confirmed the presence of exclusively IgG1 antibodies in the purified fractions (Figure 6C). Furthermore, ELISA analysis of $8.18 c 5$ cell culture supernatants indicated that only $\operatorname{lgG} 1$ antibodies, but no other isotypes localized downstream of IgG1 were produced and secreted (Figure 6D). 
A
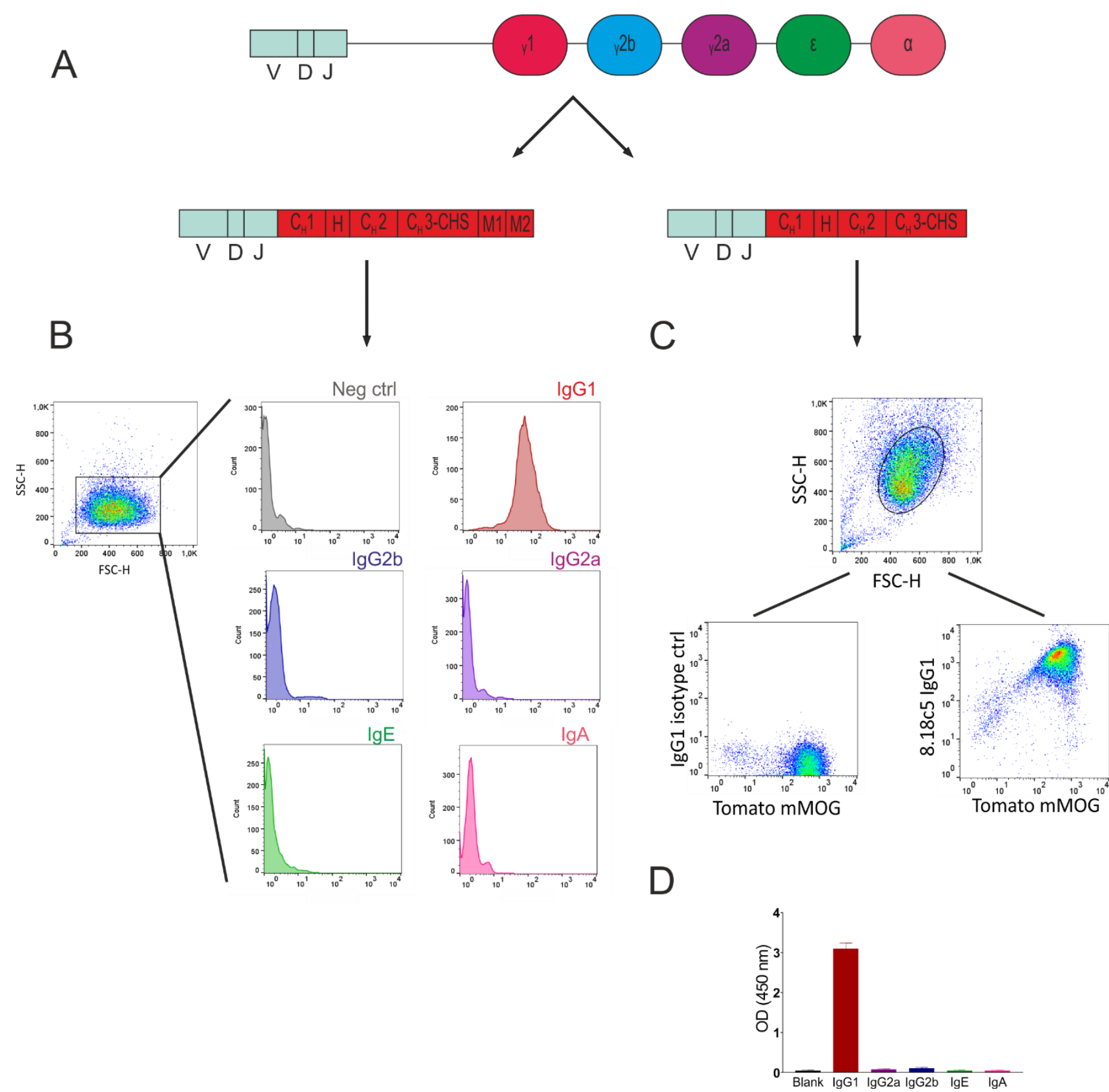

Figure 6. 8.18c5 hybridoma expresses at the membrane and releases in the supernatant antibodies of the IgG1 isotype: A) Schematic overview of the genomic organization of 8.18c5 heavy chain locus (upper), that upon transcription generates either a transcript containing membrane bounding signaling peptides (down left), or a transcript lacking these signaling peptides that leads to vesicle packaged antibodies that will be secreted (down right). B) $1 \times 10^{6} 8.18 \mathrm{c} 5$ cells were labelled with antibodies recognizing IgG1 and antibody isotypes localized downstream IgG1, or isotype control. C) FACs staining of T-mMOG cells with 8.18c5 purified antibodies. Gating strategy is showed in the upper panel. $1 \times 10^{6} 8.18 \mathrm{c} 5$ cells were stained with $0.5 \mu \mathrm{g}$ of $8.18 \mathrm{c} 5$, and PE-conjugated IgG1 or IgG1 isotype control (lower panel). D) ELISA detection of $8.18 \mathrm{c} 5$ antibodies in supernatant. $8.18 \mathrm{c} 5$ purified antibodies were tested with recombinant mMOG ELISA-coated plates $(4 \mathrm{\mu g} / \mathrm{ml})$ for $\mathrm{lgG} 1$ and downstream-localized isotypes. 


\subsubsection{Design and cloning strategy of a CRISPR-Cas9 based approach for the induction of antibody isotype switch in the $8.18 c 5$ hybridoma cell line}

Since post-translational processing of immunoglobulins such as the glycosylation pattern have a crucial role in antibody effector functions ${ }^{103}$, we aimed to induce the expression of different heavy chains directly into the 8.18c5 hybridoma. CRISPR-Cas9 based approaches demonstrated recently to be useful inducing antibody isotype switch in hybridoma cell lines ${ }^{45}$. Considering this, we designed a CRISPR-Cas9 based targeting system following the strategy of these previous studies. Cas9-containing specific gRNAs for targeting the upstream region of the first constant domain exon of the actually expressed immunoglobulin (IgG1), and upstream of the first constant domain exon of the following immunoglobulin to be expressed (IgG2b, IgG2a, IgE or IgA), would induce a genomic DNA (gDNA) double-strand break (DSB) (Figure 7). Similar to the natural CSR recombination process, the gDNA sequence found between the targeted regions would be deleted, and the Cas9-targeted regions would then be re-ligated. Consequently, the new heavy chain constant domains will be located directly after the genomic region spanning the already recombined VDJ region. Upon transcription of the locus, the transcripts will contain the sequence for the newly recombined isotype.
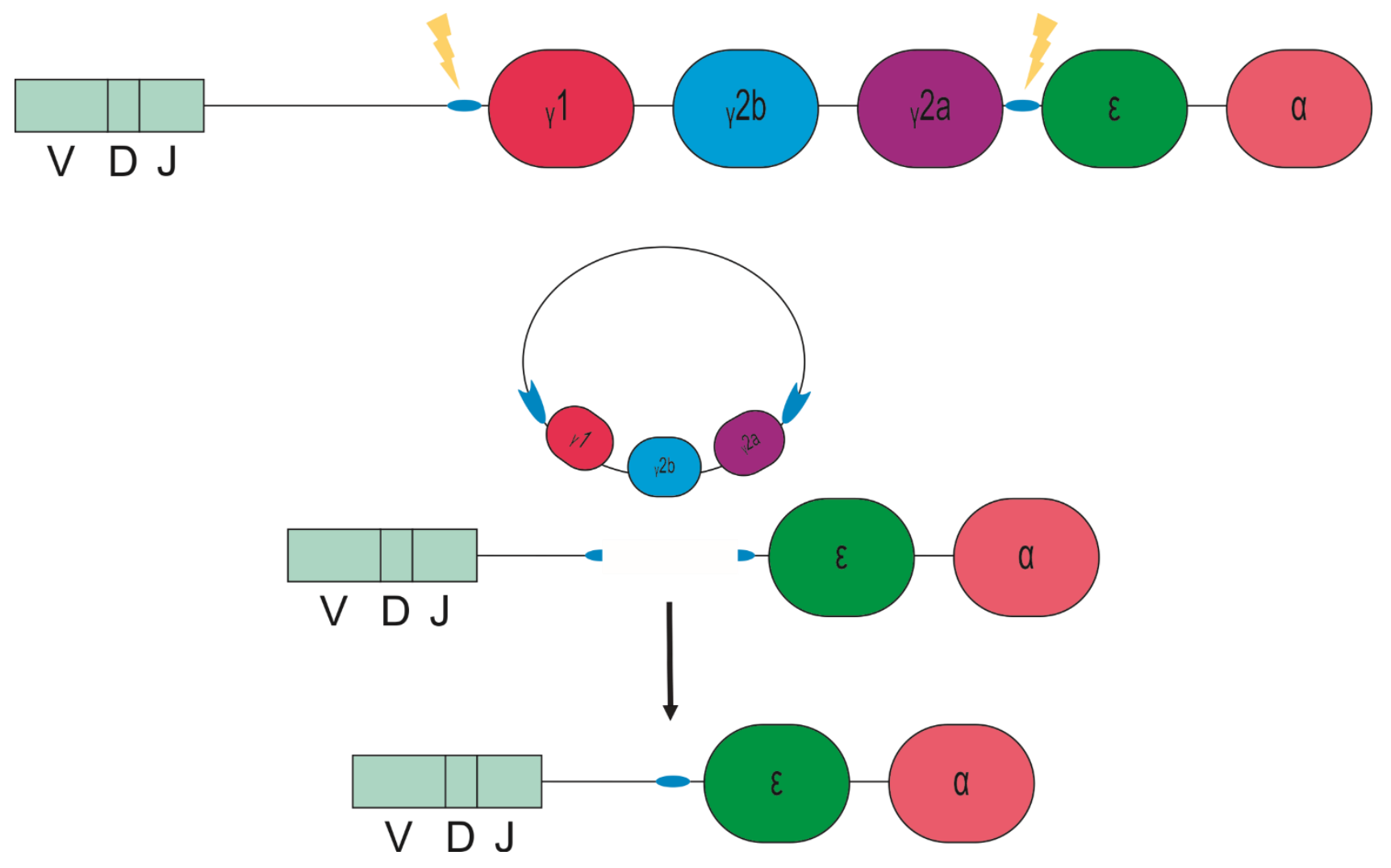

Figure 7. Emulation of CSR through CRISPR-Cas9-based strategies: Schematic overview of the CRISPR-Cas9 rational design followed in order to generate antibody isotypes localized downstream IgG1 while preserving the same antibody specificity. Generation of IgE as an example is depicted. CRISPR-Cas9 targeting of both regions inmediately located upstream IgG1 and IgE (blue dots) should lead to DSB, excluding the gDNA region located in between and leading to a rejoining of the DNA strands. In this new configuration, IgE-codifying exons will be located directly downstream the VDJ exons, therefore altering the expressed isotype but keeping the antibody specificty intact. 
Generation of MOG-specific 8.18c5 IgE from IgG1-expressing $8.18 \mathrm{c5}$ cells will be used as an example of the protocol used for the generation of different antibody isotypes. Using NCBI reference gDNA sequences from Balb/c mice, we designed primers in order to amplify a $500 \mathrm{bp}$ gDNA fragment containing the regions preceding the $\mathrm{C}_{\mathrm{H}} 1$ sequences of IgG1 and IgE (Figure $\mathbf{8 A}$ ). After cloning and sequencing of the PCR-amplified DNA fragments, different guide RNAs (gRNAs) were designed considering the existing protospacer adjacent motif (PAM) sequences within the DNA fragments (Figure 8B, top). Table 4 summarizes the sequences of the protospacers for CRISPR-Cas9-targeting upstream the IgH constant sequence of different isotypes. The selected protospacers were cloned into DNA vectors containing a puromycin resistant cassette for selection and the Cas9 protein sequence (Figure $\mathbf{8 B}$, bottom).

Of note, in order to ensure independent transcription of the different gRNAs within the targeted cells, the protospacers for the upstream region of IgG1 were cloned under the U6 promoter, and the protospacers for the upstream region of other isotypes were cloned under the $\mathrm{H} 1$ promoter. After cloning, the resulting vectors were tested with restriction enzymes, and sequenced by Sanger sequencing (Figure $\mathbf{8 C}$ ). Sequencing data confirmed the introduction of the gRNAs into the DNA constructs. 
A

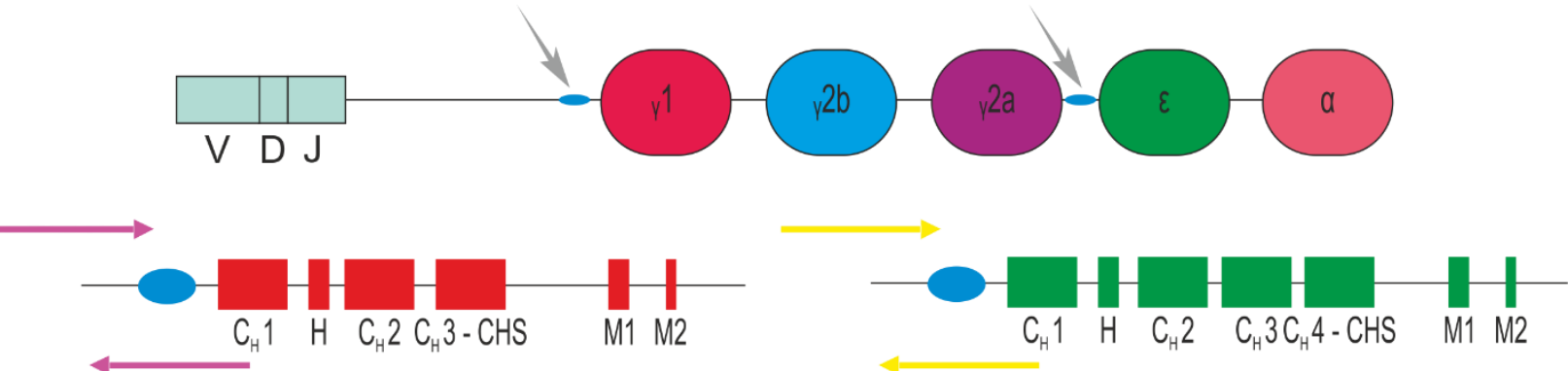

B GGCAAAGACAGGAAGGGACTGTTAGGCTGCAGGGATGTGCCGACTTCAATGTGCTTCAGTATTGTCCAGATTGTGTGCAGCCA CCATACTGGAAAG CAG GACATTGATAGGCCCAAATGTTGGATATCACCTACTCCATGTAGAGAGTCGGGGACATGGGAAGGGTGCAAAAGAGCGGCCT CTAGAAGGTTTGGTCCTGTCCTGTCCTGTCTGACAGTGTAATCACATATACTITITCTIgtagCCAAAACGACACCCCCATCTGT

ATAATGGCTTCCAGTCTGCCACCCAGCTCTCATGCTTCATTTCTGGACATTCCCTAGATGGTGTCACTGTCACCTGGTCTAAAGGA CAGACAGGAGATACCTCACACATATCCACAAAATTTCCCAATCAAGAAAGAGGGCAAGTTTGCCTCTTTATCCAAACTTGTGCCC AGCTCTCCTGGAAGCCCCTGTGCTAAGCCAGCGAGGGGCAGTGAGTGAGCAAAGCCTGGCGGGGTGTAAGGAATCAGGGAG CTCCCTAGGTCTGTGITTGGGTTTAGAGAATAAAGACAAGACCCAGAATGAATCTAACCATCTGTCTCCTAGACTGGAATGGGG TCCCCAGAG CCCTGCTCCTGTCACAGCTGCCOTIAATCAGTTCCCCATGCTGCAGGCATGCAGTGATATAAATAAGTCTAACCTAG GTCCTTCCTTTCTCacagCCTCTATCAGGAACCCTCAGCTCTACCCCTTGAAGCCCTGTAAAGGCACTGCTTCCATGACCC ATCCACTGGCCCCTGGATCTGCTGCCCAAACTAACTCCATGGTGACCCTGGGAT GCCTGGTCAAGGGCTATTTCCCTGA

gRNA-IgE-Ps2

gRNA-lgG1-Ps3

GAGAGTCGGGGACATGGGAA

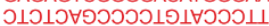

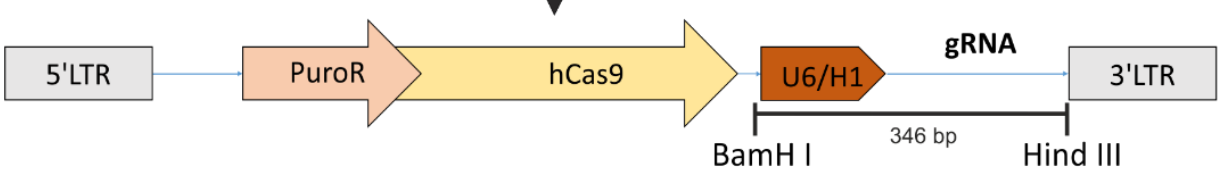

C

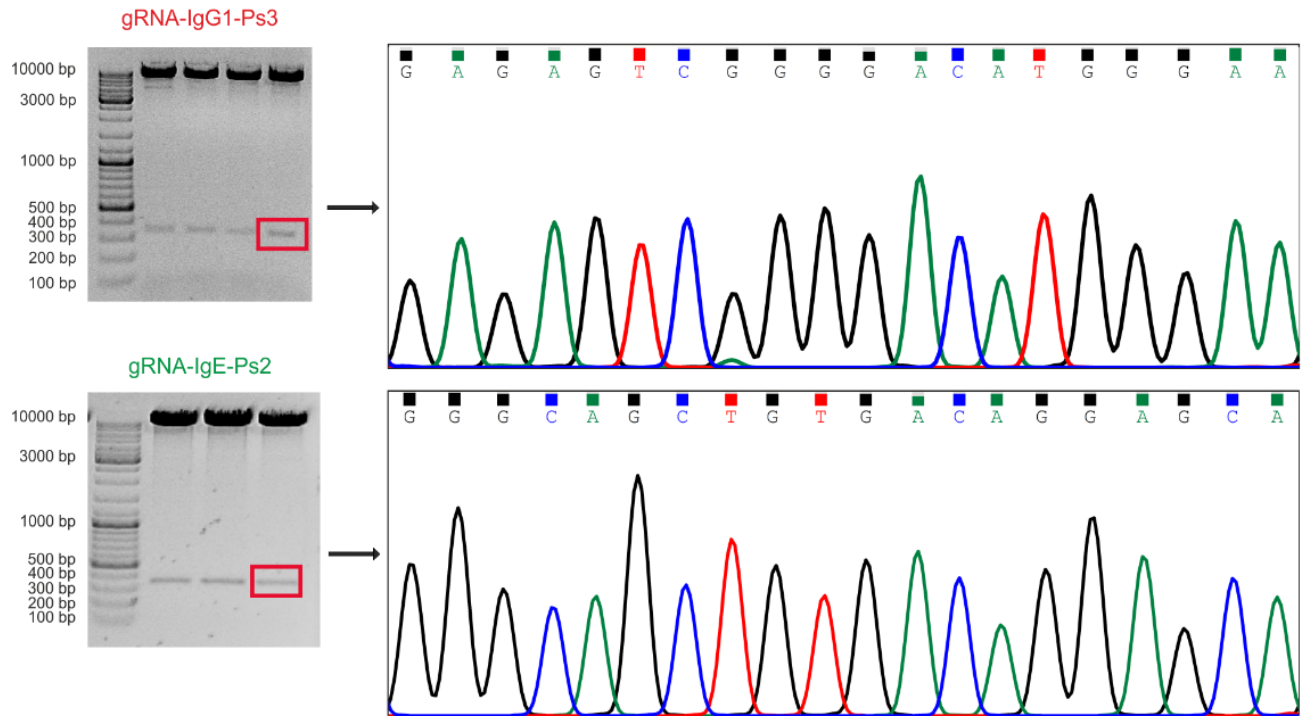

Figure 8. Cloning strategy for the generation of 8.18c5 IgE. A) PCR amplification of the gDNA containing the upstream regions preceding IgH of IgG1 and IgE. Sequences from NCBI databank were used for primer design. 25 nucleotides-long primers carrying restriction enzymes sites were used for PCR amplification. Subsequent gDNA fragments were cloned into the cloning vector PJET 1.2/blunt (ThermoFisher) and sequenced by Sanger Sequencing. B) Design of oligonucleotides for protospacer cloning into Cas9-containing vectors. 20 nucleotides-long oligonucleotides adjacent to GXX sequences (PAM regions) were designed, annealed and phosphorylated, and cloned into cloning vectors containing Cas 9 under either the $\mathrm{U} 6$ or the $\mathrm{H} 1$ promoter. C) Cloning was confirmed using BamHI and Hindlll restrictions sites and gel electrophoresis. Introduction of the protospacers into the Cas9-containing DNA vectors was confirmed by Sanger sequencing. 
Table 4. Protospacer sequences for upstream targeting of the IgH regions. Sequences of the protospacers used for CRISPR-Cas9 targeting of the 8.18c5 and Z2 hybridoma cell lines are indicated.

\begin{tabular}{|c|c|c|c|}
\hline \multicolumn{4}{|c|}{ Protospacer sequences for Cas9 targeting upstream gDNA sequences of antibody heavy chain locus } \\
\hline \multicolumn{2}{|r|}{ IgG1 } & \multicolumn{2}{|r|}{$\operatorname{lgG} 2 \mathrm{~b}$} \\
\hline Ps1 & & Ps1 & \\
\hline Forward & caccGCCTCAGGTCTCATCTGGTC & Forward & tcccCACAGTGCAAGCTCTGGAGG \\
\hline Reverse & aaacGACCAGATGAGACCTGAGGC & Reverse & aaacCCTCCAGAGCTTGCACTGTG \\
\hline Ps2 & & Ps2 & \\
\hline Forward & caccGAGGTATAAGAAGTTTAACAG & Forward & tcccGGTCACAGTGCAAGCTCTGG \\
\hline Reverse & aаacCTGTTAAACtTCTTATACCTC & Reverse & aaacCCAGAGCTTGCACTGTGACC \\
\hline \multicolumn{4}{|l|}{ Ps3 } \\
\hline Forward & caccGAGAGTCGGGGACATGGGAA & & \\
\hline Reverse & aaacTTCCCATGTCCCCGACTCTC & & \\
\hline \multicolumn{2}{|r|}{ IgE } & \multicolumn{2}{|r|}{ IgA } \\
\hline Ps1 & & Ps1 & \\
\hline Forward & tcccGAATAAAGACAAGACCCAGA & Forward & tcccGTCGGAGCTGACCAACAGTTC \\
\hline Reverse & aaacTCTGGGTCTTGTCTTTATTC & Reverse & aaacGAACTGTTGGTCAGCTCCGA \\
\hline Ps2 & & Ps2 & \\
\hline Forward & tcccGGGCAGCTGTGACAGGAGCA & Forward & tcccgCTGCATTGTGTACCCGCAGT \\
\hline Reverse & aaacTGCTCCTGTCACAGCTGCC & Reverse & aaacACTGCGGGTACACAATGCAGc \\
\hline
\end{tabular}

\subsubsection{High cleavage efficiency of the target sequence is achieved with specific guide RNAs}

After introduction of the protospacers in the Cas9 vectors, we tested these DNA construct for cleavage of the target sequences using a truncated-Cherry reporter assay (Figure 9A). The target sequences for each gRNA were cloned in the middle of a Cherry sequence, which is expressed under a CAG promoter (Figure 9A, middle and bottom). In this configuration, the presence of the target DNA sequence impairs Cherry expression, limiting its fluorescent emission. Upon cleavage of the target sequence by Cas9, the homology regions of Cherry will be able to recombine and lead to a translated functional Cherry protein. Therefore, the increase of Cherry fluorescent signal will provide insights into the cleavage efficiency of the target sequence by the designed guide-RNAs. HEK cells were transfected with CAG-Cherry DNA vectors containing the target sequences for IgG1 and IgE, and Cas9 DNA vectors expressing guide-RNAs for IgG1, IgE or no gRNAs (zero). Fluorescently labelled HEK cells were visible 24 hours after lipofectamine transfection (Figure 9B). The enhancement of Cherry fluorescence was assessed by FACs 48 hours after lipofectamine transfection (Figure 9C). Measurement of the Cherry mean fluorescence intensity (MFI) in transfected HEK cells showed that, in comparison to the vectors containing no guide-RNAs, Cas9 vectors expressing specific gRNAs for the target sequence enhanced the Cherry MFI for both IgG1 and IgE. These results indicate that our Cas9-gRNAs system is able to cleave the target sequence. The protospacer sequences indicated in Table $\mathbf{4}$ were cloned into Cas 9 vectors, and their efficiency was tested using an identical approach. Guide-RNAs showing high cleaving efficiency of the target sequences were selected for the upcoming experiments. 
A

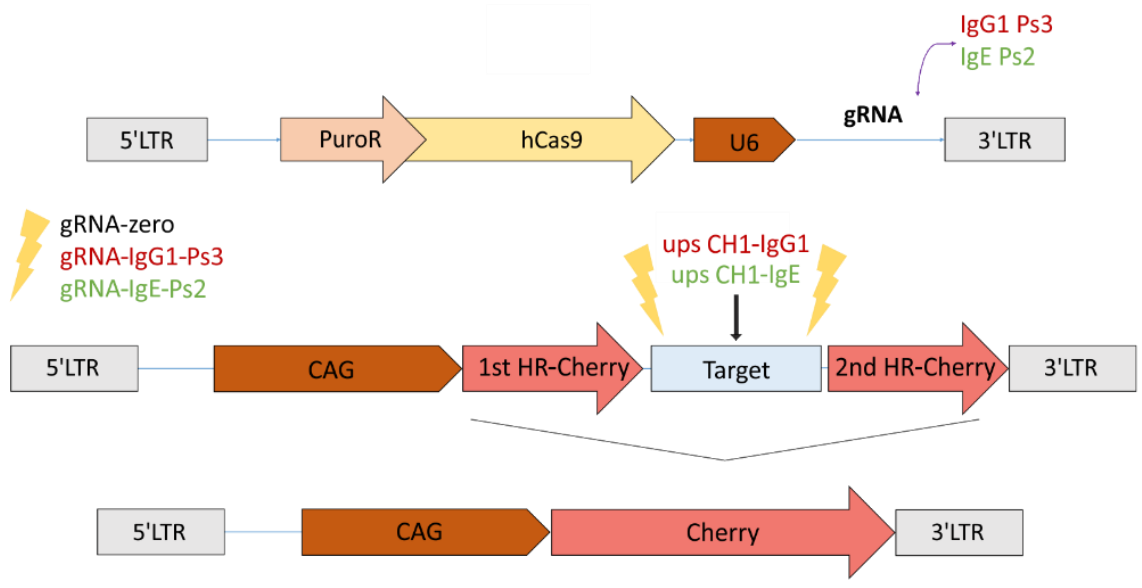

B
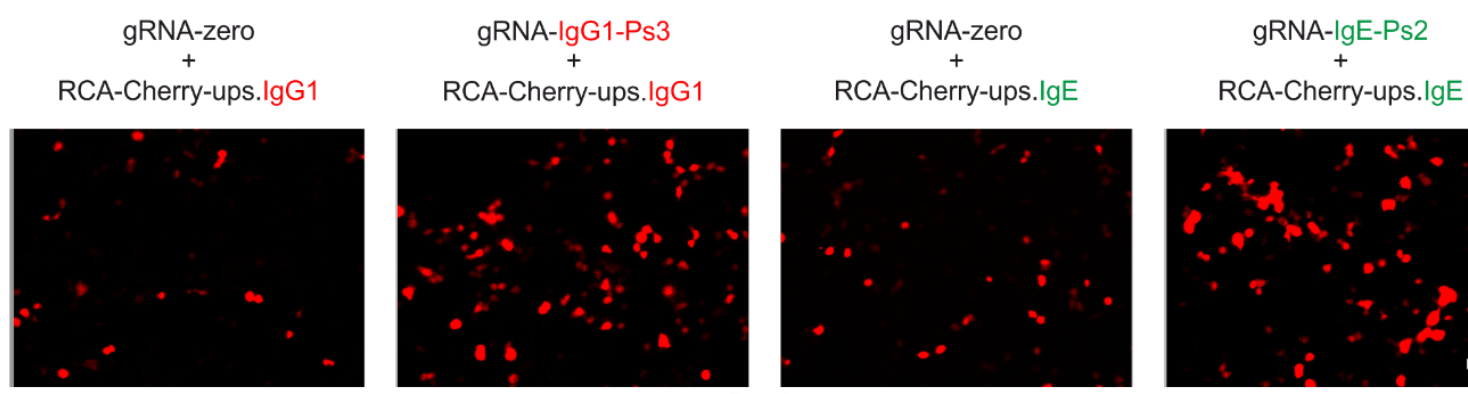

C

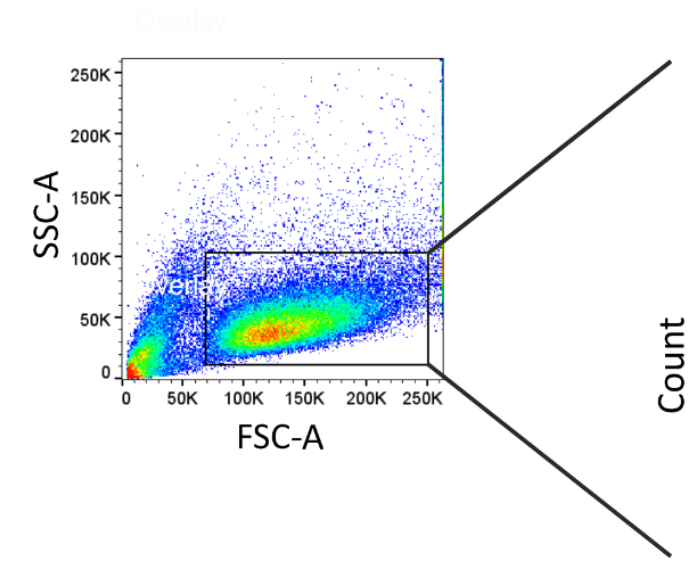

RCA-Cherry-ups.IgG1
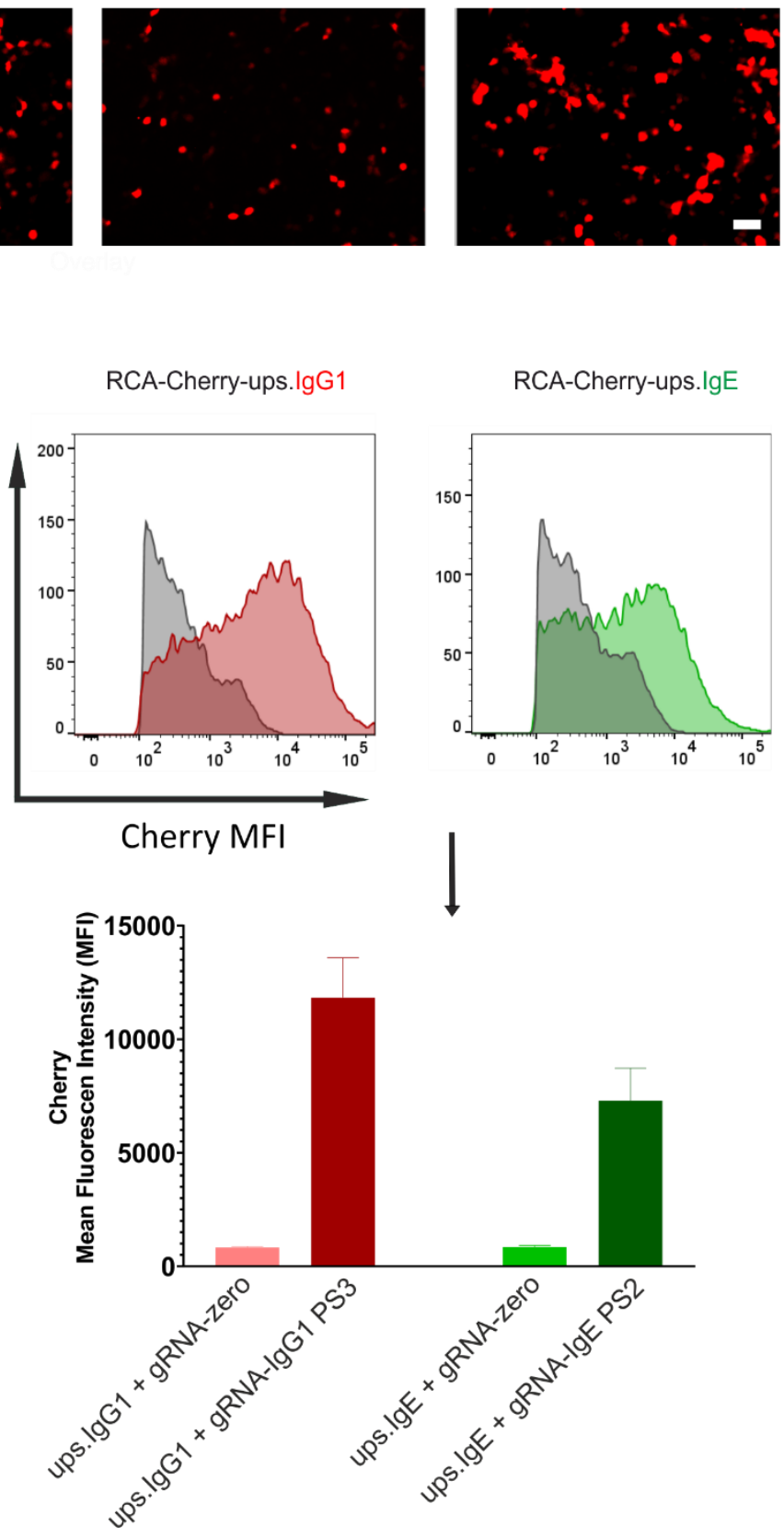
Figure 9. Confirmation of Cas9 cleavage efficiency with a split-cherry recombinant assay. A) Schematic overview of the Recombinant Cherry Assay (RCA). Upon transcription of the construct, functional gRNAs and Cas9 will cleave their target sequences, allowing the recombination of the two homologous cherry regions and leading to an enhancement of Cherry fluorescence. B) $10^{5} \mathrm{HEK}$ cells were transfected using a lipofectamine-based approach with $6 \mu \mathrm{g}$ of DNA containing Cas 9 and protospacers for targeting the upstream region of IgGI and IgE. Cherry intensity was evaluated by fluorescence microscopy (left; Scale bar, $200 \mu \mathrm{m}$ ). C) Cherry MFI was analysed by FACs. Representative MFI counts of Cherry intensity for protospacer targeting the upstream region of IgG1 (red) and IgE (green) (right). MFI quantification of Cherry intensity upon transfection of protospacers for IgG1, IgE or control Cas9 vectors (bottom).

\subsubsection{CRISPR-Cas9 targeting upstream the IgH constant regions of IgG1 and IgE lead to isotype switch in the $8.18 \mathrm{c} 5$ hybridoma}

The DNA constructs containing the protospacers for targeting the sequences preceding IgH constant domains of IgG1 and IgE were introduced into the $8.18 \mathrm{c} 5$ hybridoma cell line by electroporation. After one day, the transfected $8.18 \mathrm{c} 5$ cells still expressed IgG1 in the membrane but not IgE (Figure 10A left). After 4 days we were able to detect two different populations which expressed either IgG1 or IgE at the cell membrane (Figure $10 \mathrm{~A}$ middle). We isolated single positive IgE-expressing cells by cell-sorting, and Ig membrane expression was further evaluated 3 days later by FACs, which revealed the expression of only IgE at the cell membrane but not IgG1 (Figure 10A right), or any other isotype located downstream IgG1 in addition (Figure 10B). Using the same protocol but with Cas9 vectors containing the protospacer 1 for the upstream region of IgG2b heavy chain instead (Table 4), we could additionally isolate a population expressing exclusively IgG2b at the cell membrane (data not shown). 
A
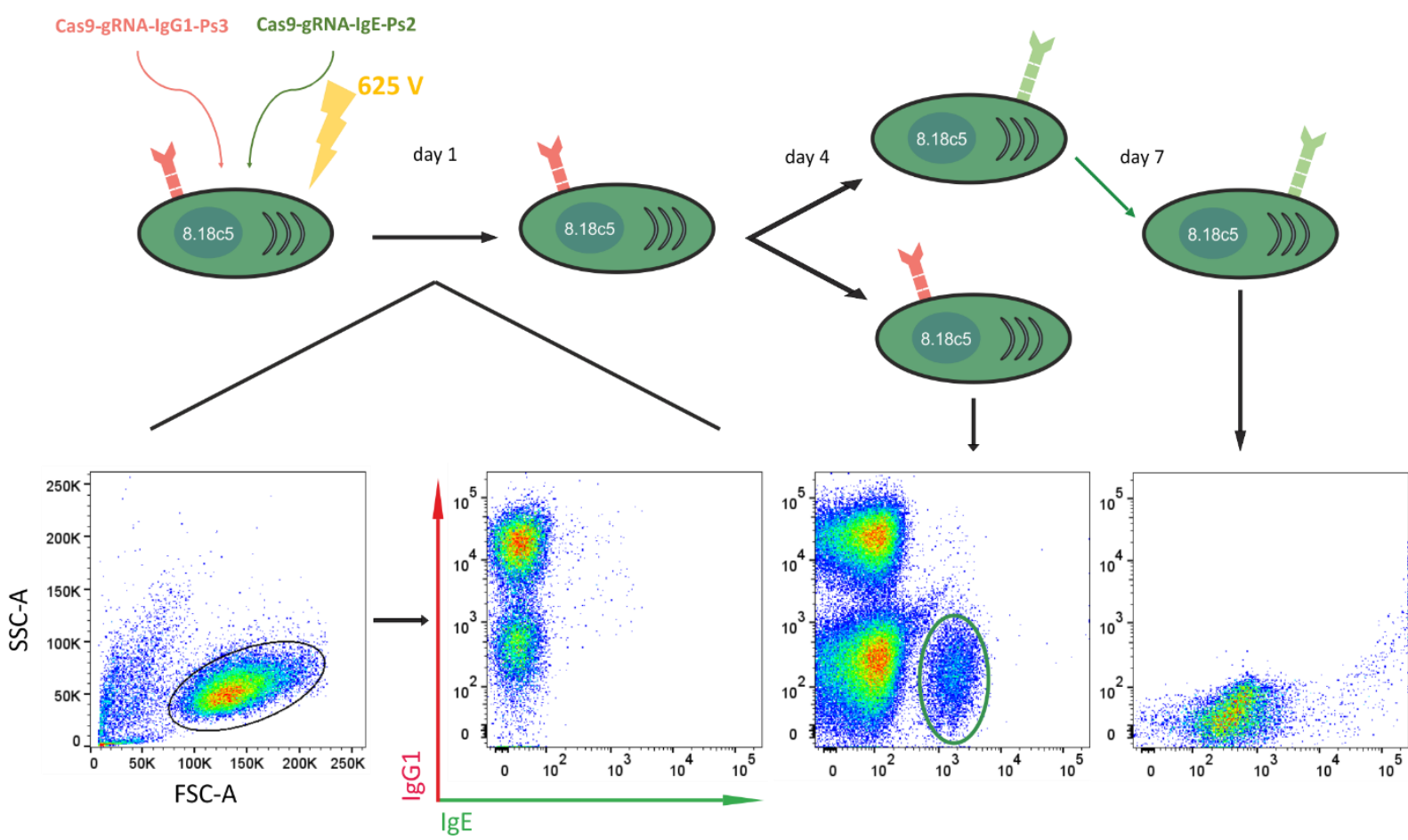

B
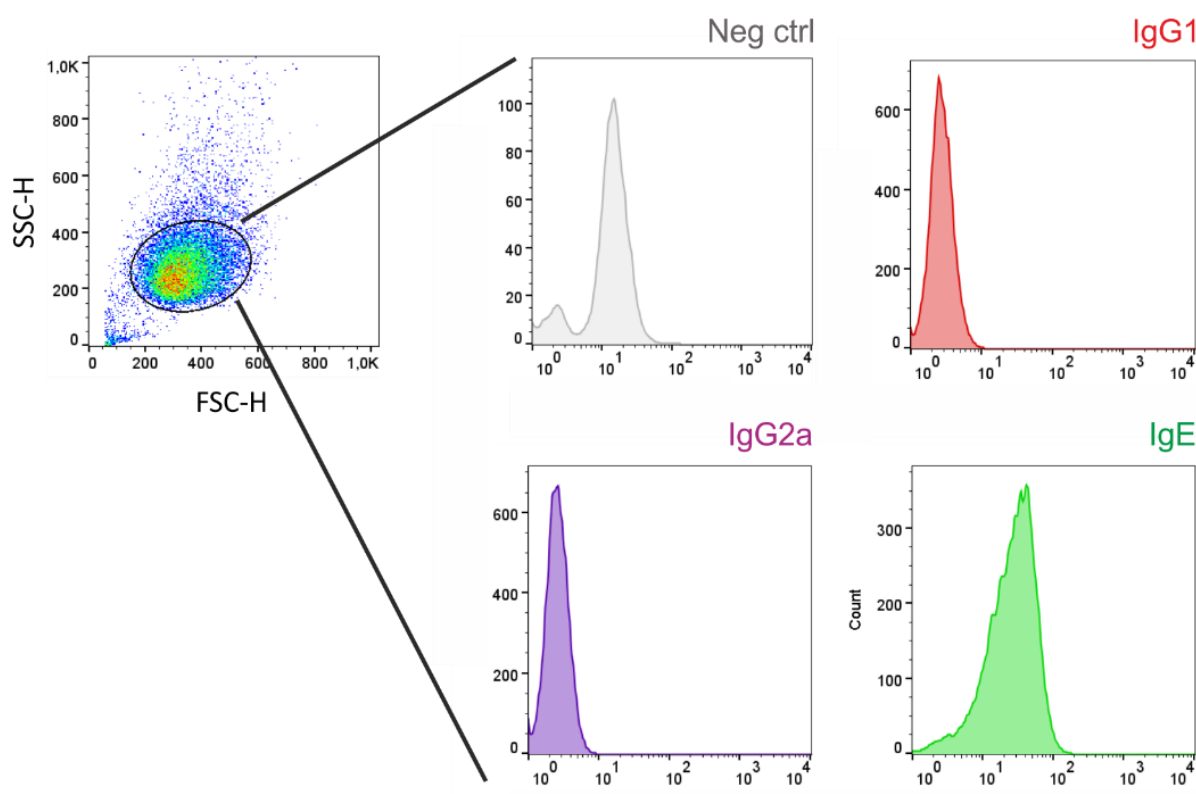

$\lg G 2 b$
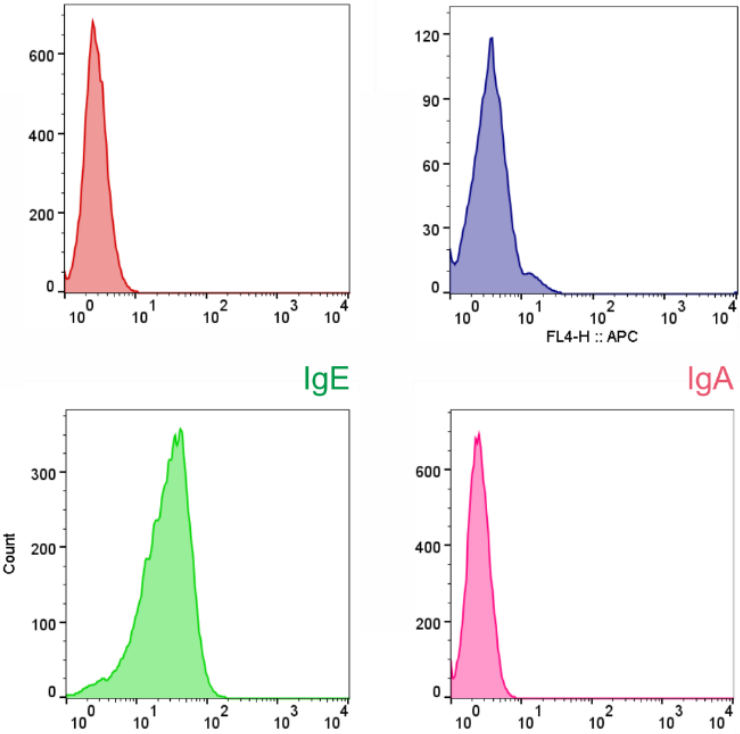

Figure 10. CRISPR-Cas9 induced isotype switching of 8.18c5 leads to the expression of a different antibody isotype at the membrane level. A) $2 \times 10^{5} 8.18 \mathrm{c} 5$ cells were electroporated with Cas9-containing DNA vectors and protospacers for the upstream region of IgG1 and a different isotype located downstream. An example for the generation of IgE is depicted (upper). Transfected cells were analysed during the following days after electroporation and single-positive cells for IgE were sorted after 4 days. Stable expression of IgE at the membrane level was confirmed 3 days later (lower). B) FACS analysis of IgE positive cells for immunoglobulin membrane expression. $1 \times 10^{6}$ cells were stained with biotin-conjugated antibodies for IgG1, IgG2b, IgG2a, IgE or IgA; and revealed with st-APC. 


\subsubsection{Selected isotype switched hybridoma clones release MOG-specific antibodies of the desired isotype into the supernatant}

Since membrane expression of a new antibody isotype in the 8.18c5 hybridoma cell line does not necessarily imply the production of soluble antibodies, we wanted to characterize whether the new cell lines were secreting antibodies into the supernatant, and in that case, which antibody isotype was being released. After sorting of a single positive $8.18 \mathrm{c} 5$ hybridoma cell population expressing the new immunoglobulin in the membrane, the isolated hybridoma cells were subcloned using limiting dilution protocols (Figure 11A). Dilution of cells until arriving to a concentration of one cell per well allowed us to ensure that the new hybridoma cell line derives from a single cell, but also enables us to analyse high numbers of different clones at the same time by ELISA. Analysis of the supernatants by ELISA revealed that, while some clones were still able to produce IgG1, other clones were producing specifically the new immunoglobulin (Figure 11B), which were expanded and cultured for antibody production (Figure 11C). Supernatants were purified by affinity chromatography and the eluted fractions were quantified. The purified antibodies were then evaluated by gel electrophoresis and western blot (Figure 11D). Gel electrophoresis revealed a molecular weight increase of the heavy chain of IgE antibodies compared to IgG1, given the extra C domain found in the IgE structure (Figure 11D upper). Western blot showed immunoreactivity for 8.18c5 IgE samples in presence of anti-IgE antibodies but not in the presence of anti-IgG1 antibodies (Figure 11D bottom). Next, we addressed whether the new antibodies were able to bind to their target protein, mMOG. ELISA assays in rmMOG-coated plates confirmed the binding of the antibody to MOG, as well as the presence of only antibodies of the IgE isotype (Figure 11E). The purified antibodies were further evaluated by FACs analysis using T-mMOG cells, confirming that the new antibodies were exclusively of the IgE isotype and that they recognized MOG expressed at the surface of TmMOG cells (Figure 11F).

Following this approach, it was possible to establish $8.18 \mathrm{c} 5$ hybridoma cell lines producing IgE, but also $8.18 \mathrm{c5}$ hybridoma cell lines producing IgG2b. Purified antibodies derived from IgG2b-expressing cells showed specificity for mMOG (Figure 12). Sufficient quantities of 8.18c5 IgE and 8.18c5 IgG2b were produced in order to verify the antigen specificity and the correct isotype of the antibodies, but also to perform in vivo experiments. 

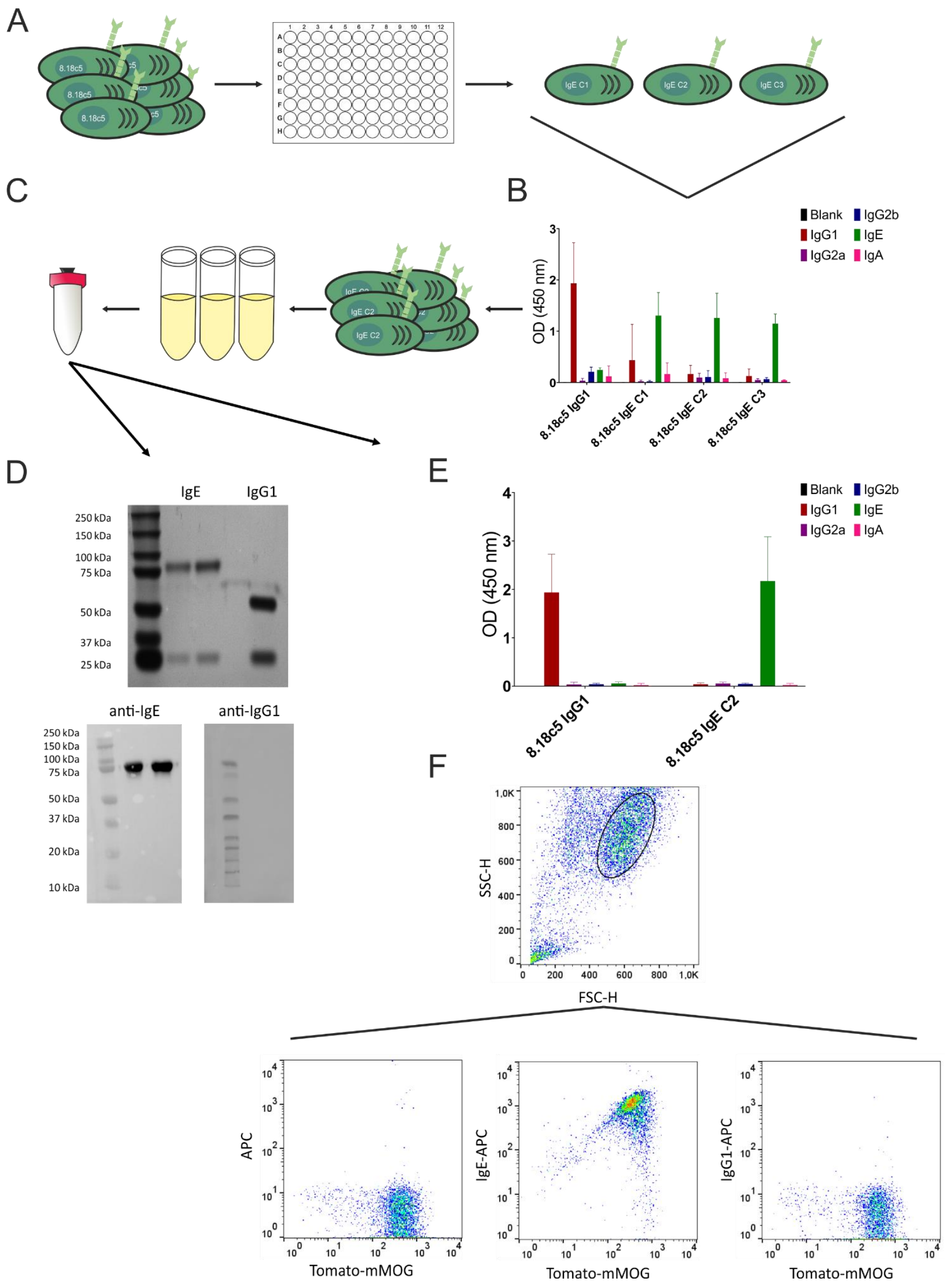
Figure 11. Newly generated $8.18 \mathrm{c} 5$ cell lines secrete antibodies with different isotypes while preserving the antigen specificity. A) 8.18c5 IgE-expressing cells were diluted until a 5 cell/ml concentration for single cell subcloning in 96 wellplates. B) Supernatant from IgE-expressing subclones was tested by rmMOG ELISA for antibody isotype characterization. C) Clones expressing exclusively IgE were isolated and expanded for antibody production in PFHM-II. Supernatants were collected and purified by affinity chromatography (GE Healthcare). D) Purified proteins were tested by gel electrophoresis (upper) and Western Blot (lower). $1 \mu \mathrm{g}$ of protein was denatured at $90^{\circ} \mathrm{C}$ in $\beta$-mercaptoethanol. Electric separation of proteins was performed at $100 \mathrm{~V}$. For molecular weight characterization, agarose gels were stained with cromassie blue. For Western Blot, eluted proteins were tested with anti-IgE and anti-IgG1 antibodies. E) ELISA analysis of 8.18c5 IgG1 and IgE antibodies. Purified antibodies from 8.18c5 IgG1 and IgE expressing cell lines were tested with recombinant $\mathrm{mMOG}$ ELISA-coated plates $(4 \mu \mathrm{g} / \mathrm{ml})$ for $\operatorname{lgG1}$ and downstream-localized isotypes. F) FACs staining for antibody isotype confirmation. $1 \times 10^{6} \mathrm{~T}-\mathrm{mMOG}$ cells were stained with $1 \mu \mathrm{g}$ of purified antibodies. Antibody isotype was tested with antiIgE and anti-IgG1 antibodies.

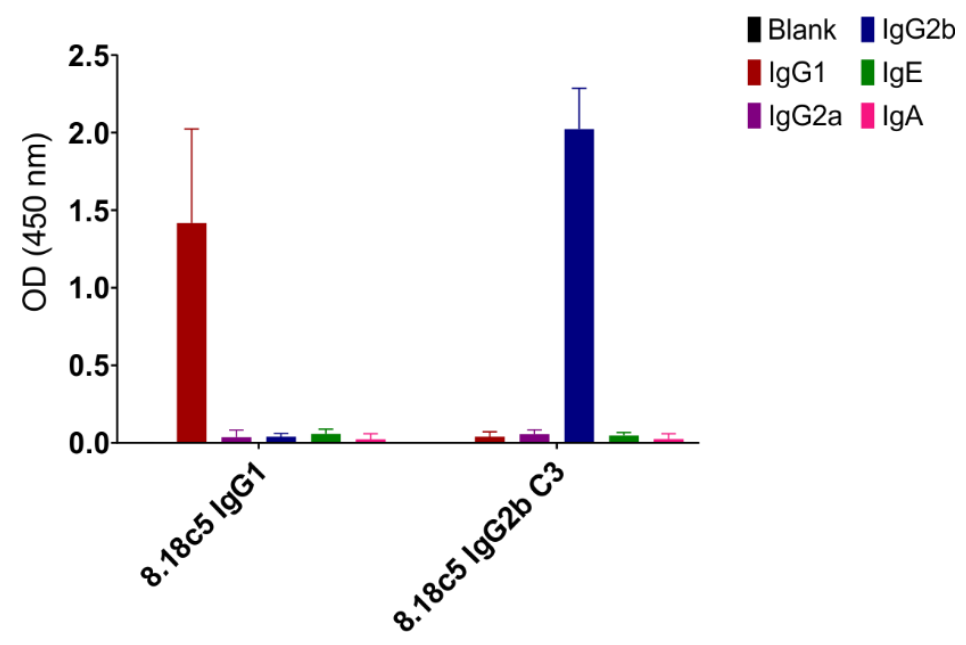

Figure 12. Characterization of affinity-chromatography purified antibodies from IgG2b expressing $8.18 \mathrm{c} 5$ clones. ELISA analysis of $8.18 \mathrm{c} 5 \lg \mathrm{Ig} 1$ and IgG2b antibodies. Purified antibodies from 8.18c5 IgG1 and Ig2b expressing cell lines were tested with recombinant mMOG ELISA-coated plates $(4 \mu \mathrm{g} / \mathrm{ml})$ for $\mathrm{IgG1}$ and downstream-localized isotypes.

\subsubsection{CRISPR-Cas9 allows the generation of different antibody isotypes in the $Z 2$ hybridoma}

The different aforementioned approaches were also used in order to switch the isotype of the produced antibodies in the $\mathbf{Z 2}$ hydridoma cell line. This hybridoma is known to produce MOG-specific autoantibodies of the IgG2a isotype ${ }^{104}$, which also binds to the FG-loop of MOG, but the recognized epitope by Z2 is not completely identical to the one recognized by $8.18 c 5^{105}$. Similar to $8.18 \mathrm{c} 5$, the $\mathrm{Z2}$ hybridoma cells expressed only one type of immunoglobulin isotype at the cell surface (Figure 13). The characterization of the $Z 2$ hybridoma, as well as the switch to different antibody isotypes using the CRISPR-Cas9 system, were performed in our department in a master's project ${ }^{106}$. Targeting of the Z2 hybridoma with Cas9-containing gRNAs for the IgH upstream sequences of IgE and IgA led to the generation of different hybridoma cell lines expressing one of these two immunoglobulins. These Z2-derived cell lines secreted antibodies into the supernatant exclusively of the isotype they were switched to, and these antibodies were also specifically binding to $\mathrm{mMOG}^{106}$. 


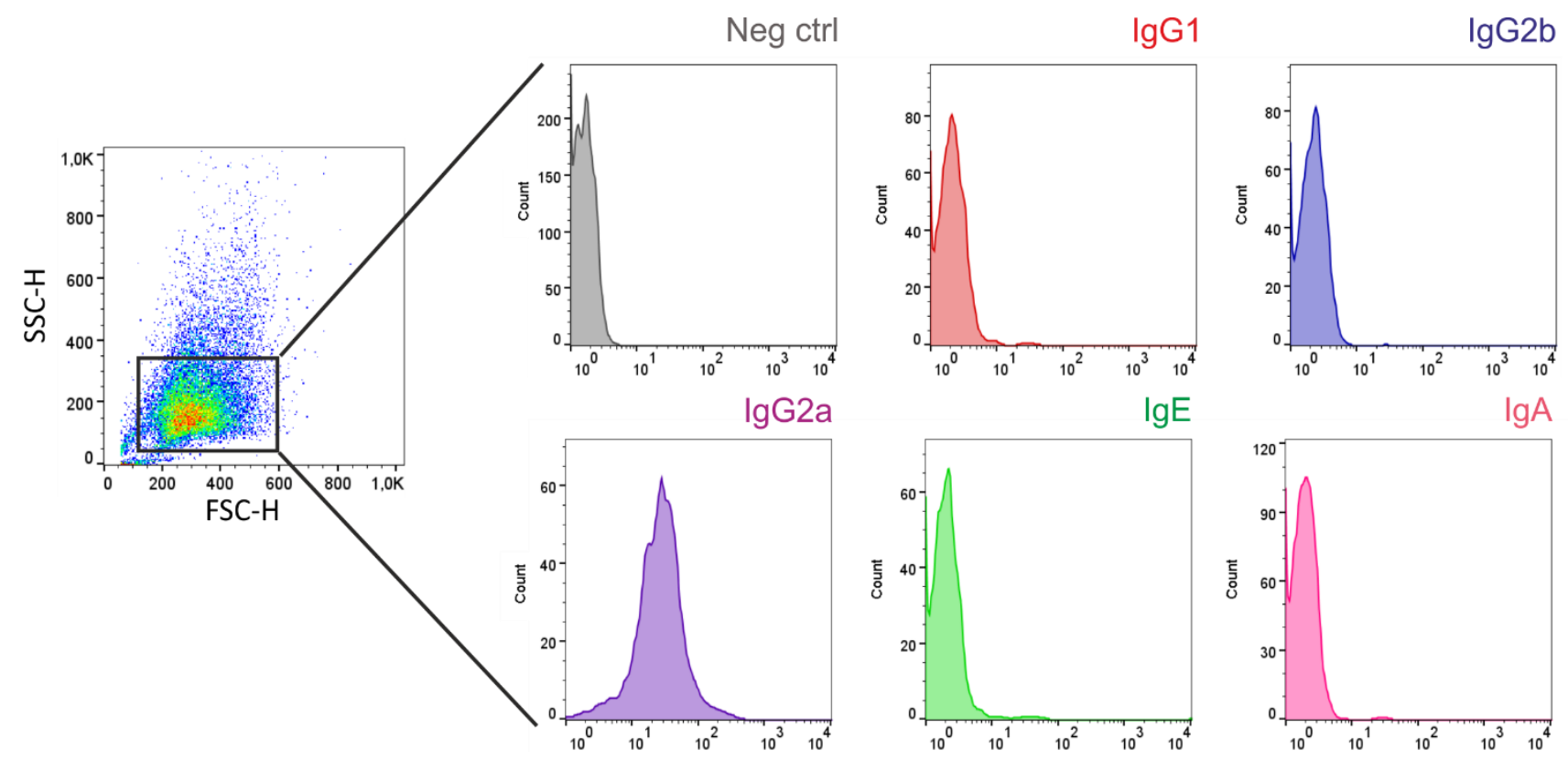

Figure 13. Z2 hybridoma cells express exclusively antibodies of the IgG2a isotype. FACs staining of Z2 for membrane immunoglobulin expression characterization. $1 \times 10^{6}$ cells were stained with biotin-conjugated $\operatorname{lgG} 1, \lg$ 2 $\mathrm{b}, \operatorname{lgG} 2 \mathrm{a}$, IgE or IgA and revealed with st-APC. Gating strategy in the left side. MFI results of immunoglobulin membrane expression are showed in the right panel.

To sum up, using our CRISPR-Cas9 based approach we could generate $8.18 \mathrm{c} 5$ cell lines producing MOG-specific antibodies of the IgE and IgG2b isotype, in addition to IgG1 antibodies produced by the original 8.18c5 hybridoma'cells. Furthermore, CRISPR-Cas9 induced isotype switch in the Z2 hybridoma, which originally produce MOG-specific IgG2a antibodies, led to the expression and production of soluble IgE and IgA antibodies. Chromatography-affinity purified antibodies derived from the different Z2 and 8.18c5 hybridoma cell lines were tested for isotype characterization and reactivity against MMOG in the T-mMOG cell line (Figure 14A and 14B). All the purified antibodies derived from these switched hybridoma cell lines contained only one type of antibody isotype. Our results show that production of every antibody isotype found downstream of the IgG1 genomic constant domain while preserving antibody specificity is possible using a CRISPR-Cas9 based approach. Large-scale production of the antibody isotypes depicted in figure 11 was undertaken in order to be able to perform in vivo experiments in the EAE model. 

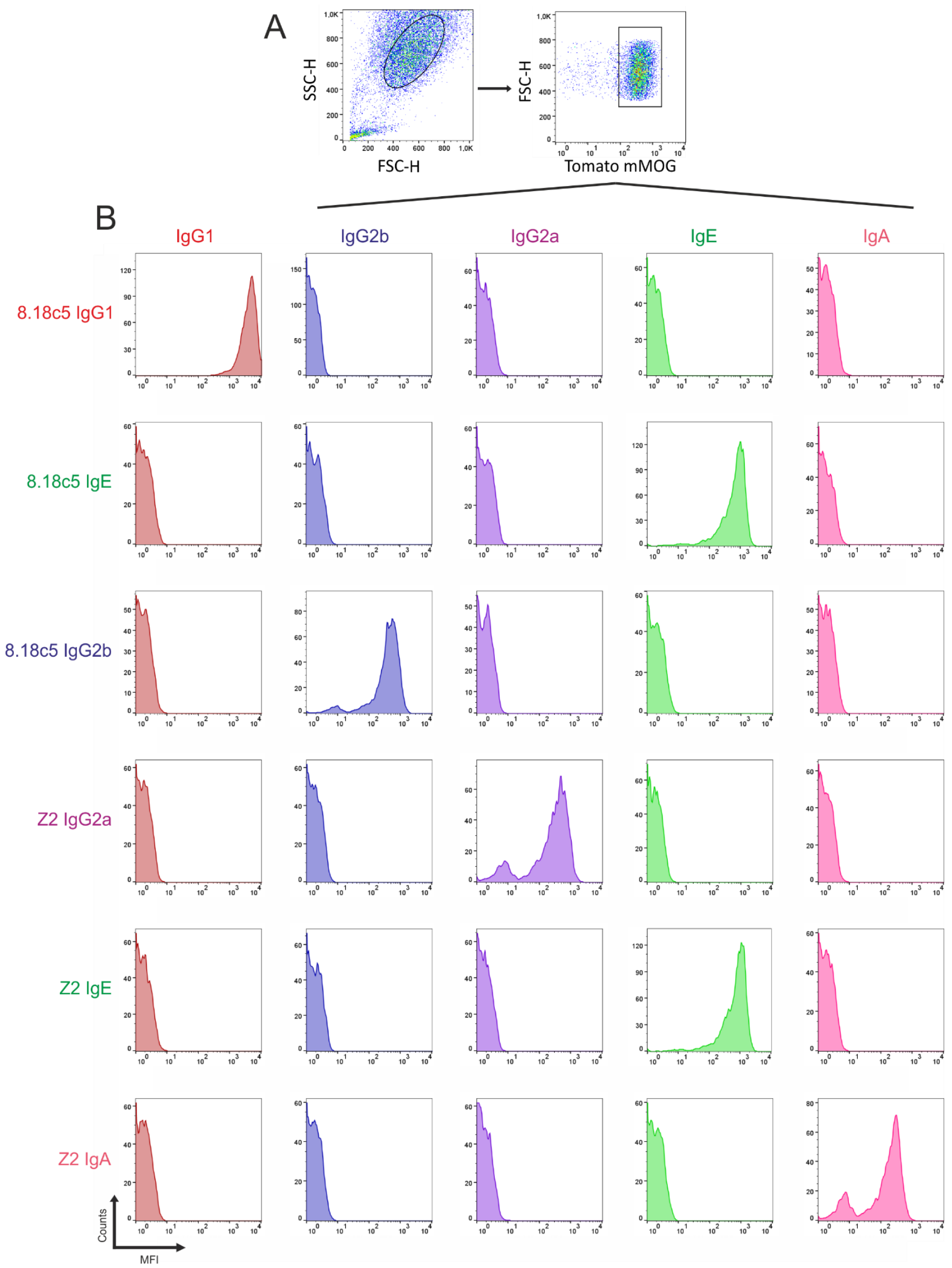
Figure 14. Characterization of newly generated purified antibodies isotypes preserving the same specificity from $8.18 \mathrm{c} 5$ and $Z 2$ hybridomas. FACs staining of T-mMOG cells with purified proteins from different $8.18 \mathrm{c} 5$ and $Z 2$ cell lines. $1 \times 10^{6}$ T-mMOG cells were stained with $1 \mu \mathrm{g}$ different purified antibodies (8.18c5 IgG1, IgE and IgG2b; Z2 IgG2a, IgE and IgA). A) Gating strategy for characterization of the antibodies binding to MOG and antibody isotype. B) MFI quantification of purified antibodies for different isotypes. $1 \times 10^{4} \mathrm{~T}-\mathrm{mMOG}$ cells were stained with purified antibodies from $8.18 \mathrm{c} 5$ and $\mathrm{Z2}$ lines varying in isotype. Presence of other antibody isotypes was tested with biotin-conjugated antibodies recognizing IgG1 and antibody isotypes located downstream.

\subsection{The antibody isotype determines different clinical outcomes in the EAE model}

Previous findings from our department showed that autoantibodies accumulated within CNS antigenpresenting phagocytes, which presented antigen more efficiently to invading effector T lymphocytes ${ }^{87}$. This resulted in an acceleration of EAE onset and a more severe disease. In these studies, antibodies of the IgG1 isotype derived from the $8.18 c 5$ hybridoma were used. Since antibody effector functions are conditioned by the antibody isotype ${ }^{107-108}$, we sought to address the role of the antibody isotype in the EAE model.

\subsubsection{MOG-specific $\lg G 1$ and $\lg G 2 b$ accelerate the disease onset and enhance disease severity in the EAE model}

In order to test whether the antibody isotype has a crucial role in EAE clinical outcome, animals were immunized and antibodies were administered shortly before the beginning of the clinical symptoms. Compared to the $\operatorname{lgG} 1$ isotype control, the original 8.18c5 antibodies of the $\lg \mathrm{G} 1$ isotype accelerated disease onset and enhanced the clinical severity of EAE as expected, both when antibodies were administered systemically (intravenously, i.v.) or locally (intrathecally, i.t.) (Figure 15A). These data confirm previous results indicating that MOG-specific antibodies have a crucial role in modulating $T$ cell reactivation within the CNS rather than in the periphery ${ }^{87}$. Very similar results were obtained when $8.18 \mathrm{c} 5$ IgG2 b was administered. Compared to the $\lg \mathrm{g} 2 \mathrm{~b}$ isotype control, $8.18 \mathrm{c} 5 \lg \mathrm{g} 2 \mathrm{~b}$ accelerated disease onset and enhanced disease severity, both when administered i.v. and i.t. (Figure 15A). Interestingly, when 8.18c5 IgE was administered either i.v. or i.t. shortly before disease onset, the animals did neither show enhanced disease severity nor an accelerated disease onset (Figure 16A). Since the half-life of IgE in plasma is shorter in comparison to other isotypes ${ }^{62}$, we administered 8.18c5 IgE on three consecutive days. Triple administration of 8.18c5 IgE did also not lead to disease acceleration and increased disease severity, in contrast to both $8.18 \mathrm{c} 5 \lg G 1$ and $8.18 \mathrm{c} 5 \lg 2 \mathrm{~b}$ (Figure 17). These results show that the disease-accelerating effect of the $8.18 c 5$ antibody in active EAE is influenced by the antibody isotype.

To provide further evidence that MOG-specific antibodies exert their effects in the CNS rather than in the periphery, we addressed their effect on disease progression upon administration of different antibody isotypes in the passive transfer EAE model, where fully activated T cell blasts do not require further priming in order to invade the CNS and induce EAE. In the transfer EAE model, 8.18c5 IgG1 worsened the disease when administered i.v. compared to IgG1 isotype control, but also when administered i.t., confirming our previous 
observations concerning the modulatory effects of antibodies within the $\mathrm{CNS}^{87}$ (Figure 15B). Acceleration of disease onset and enhancement of disease severity was also observed in the transfer EAE model upon administration of $8.18 \mathrm{c} 5 \operatorname{lgG} 2 \mathrm{~b}$ both i.v. or i.t. (Figure 15B). These results further support the role of antibodies exerting their effects within the CNS, but also point at a similar role for IgG1 and IgG2b regarding diseasemodifying effects in our model. Similar to the results obtained in the active EAE model, 8.18c5 IgE did not exert disease modulatory effects in the transfer EAE model, since animals injected i.v. or i.t. showed a similar clinical outcome when administered with 8.18c5 IgE or IgE isotype control (Figure 16B). Taken together, these results indicate that antibodies exert their effects within the CNS even after the priming phase, but also that antibody disease-modifying effects are isotype dependent. 
A

intravenous

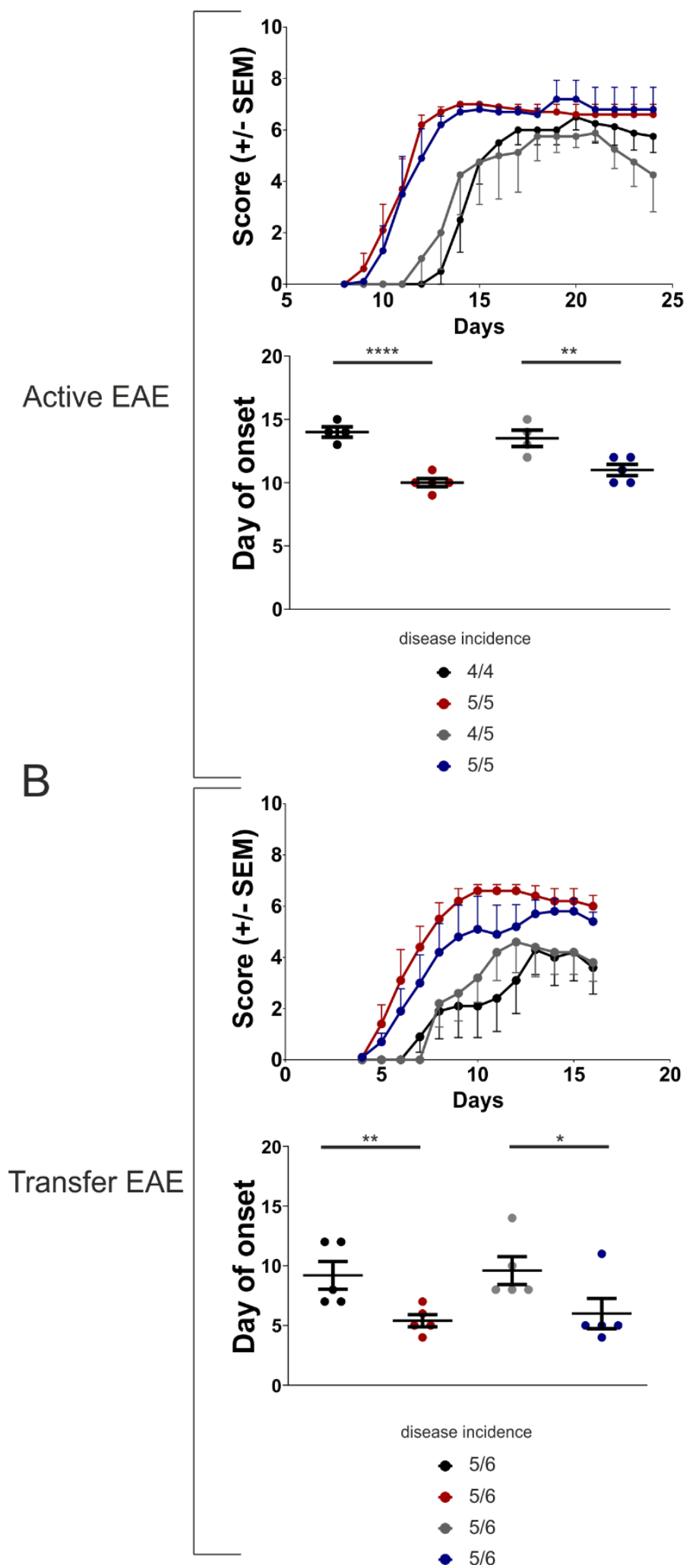

- IgG1 isotype control

- $8.18 \mathrm{c} 5 \lg \mathrm{G} 1$

- IgG2b isotype control

- $8.18 \mathrm{c} 5 \lg \mathrm{lg} 2 \mathrm{~b}$
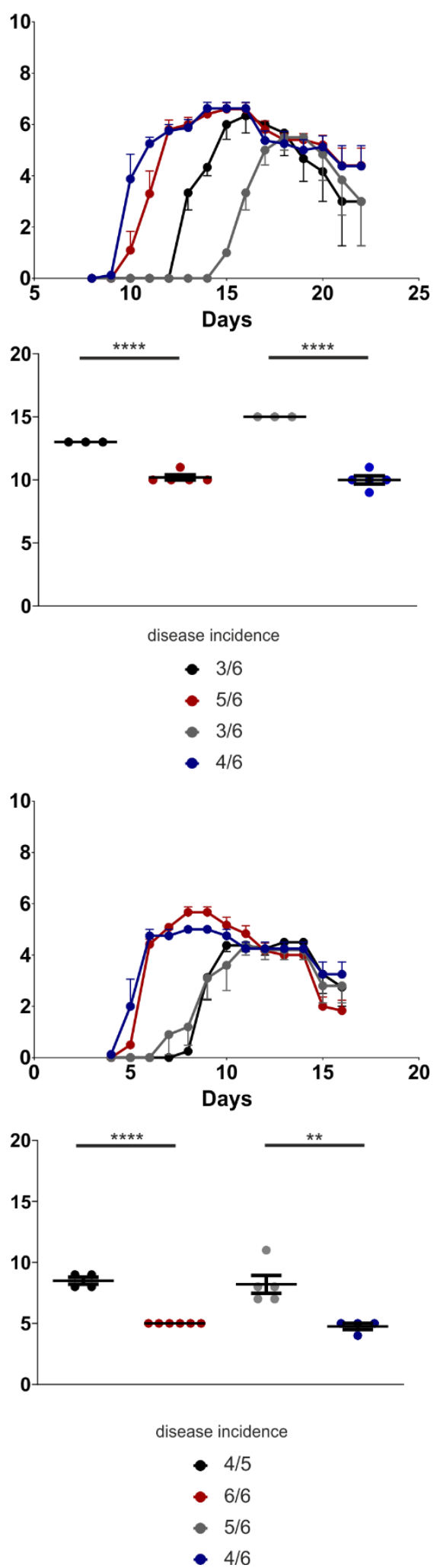

Figure 15. 8.18c5 IgG2b reproduce disease onset acceleration and disease severity effect of $8.18 \mathrm{c} 5$ IgG1. $8.18 \mathrm{c} 5$ IgG1 (red), IgG2b (blue), IgG1 isotype control (black) or IgG2b isotype control (grey) was intravenously (left) or intrathecally 
(right) injected on $\mathrm{d} 8$ after immunization with $25 \mu \mathrm{g}$ of $\mathrm{MOG}_{35-55}$ (A), or on $\mathrm{d} 2$ after $\mathrm{T}$ cell transfer (B). The clinical course of the disease (top), the day of onset (middle) and the disease incidence (down) are depicted for each model.

- IgG1 isotype control

- 8.18c5 lgG1

A

intravenous

intrathecal

- IgE isotype control

- 8.18c5 lgE
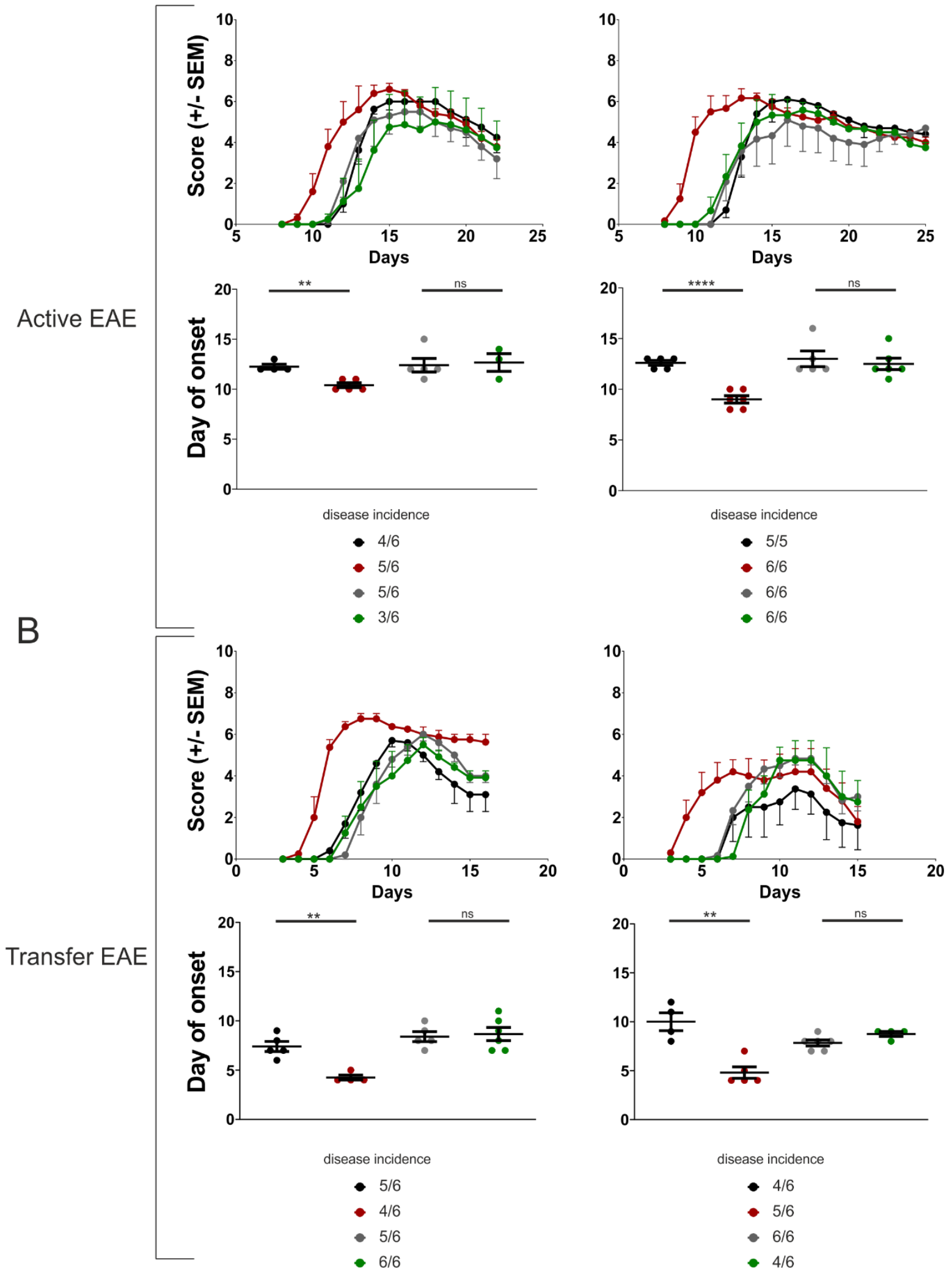
Figure 16. 8.18c5 IgE does not reproduce disease onset acceleration and disease severity effect of 8.18c5 IgG1. 8.18c5 IgG1 (red), IgE (green), IgG1 isotype control (black) or IgE isotype control (grey) was intravenously (left) or intrathecally (right) injected on d8 after immunization with $25 \mu \mathrm{g}$ of $\mathrm{MOG}_{35-55}$ (A), or on $\mathrm{d} 2$ after T cell transfer (B). The clinical course of the disease (top), the day of onset (middle) and the disease incidence (down) are depicted for each model.
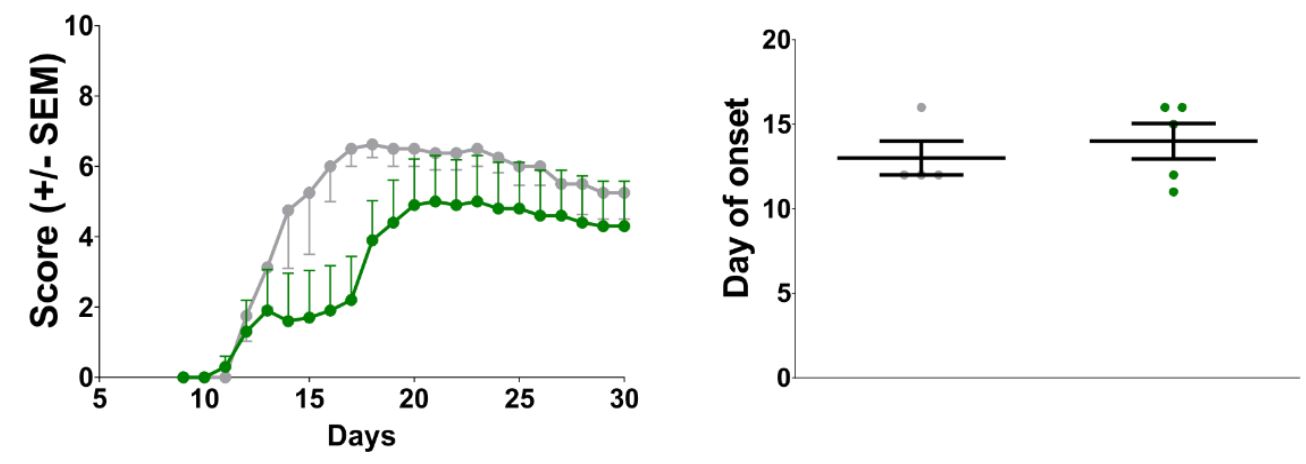

- IgE isotype control

- 8.18c5 lgE

disease incidence

- $4 / 6$

- $5 / 6$

Figure 17.Triple administration of 8.18c5 IgE does not reproduce disease onset acceleration and disease severity effect of 8.18c5 IgG1. 8.18c5 IgE (green) or IgE isotype control (gray) were administered intravenously on d8, d9 and d10 after immunization with $25 \mu \mathrm{g}$ of MOG 35 -55. The clinical course of the disease (left), day of onset (middle) and disease incidence (right) are depicted.

\subsubsection{Disease-modulatory effects on EAE mediated by MOG-specific $Z 2$ antibodies are also isotype dependent}

In our laboratory, we found that Z2 IgG2a has a similar effect on disease modulation compared to $8.18 \mathrm{c5}$ $\operatorname{lgG} 1^{109}$. Upon administration of $8.18 \operatorname{lgG} 1$ or Z2 IgG2a, the animals developed a more severe disease course and an earlier disease onset in comparison to their respective isotype controls (Figure 18). Work from a master thesis addressed the question whether CRISPR-Cas9 induced isotype switch of Z2 antibodies would have the same effect observed with the $8.18 \mathrm{c} 5$ isotypes in the active EAE model ${ }^{106}$. The results of this study showed that the animals administered with $50 \mu \mathrm{g}$ of $Z 2 \operatorname{lgG} 2$ a developed enhanced disease severity and accelerated the day of onset in comparison to IgG2a isotype control, but administration of either $50 \mu \mathrm{g} Z 2$ IgE or Z2 IgA did not reproduce this effect. These results substantiate our observations from the experiments using $8.18 \mathrm{c} 5$ antibodies of different isotypes. Summarizing all results obtained with antibodies of different isotypes derived from the $8.18 \mathrm{c} 5$ and $Z 2$ hybridoma cell lines, from the panel tested so far only antibodies of the IgG isotype were able to modify the EAE disease course, but not antibodies of the IgE or IgA isotype. 

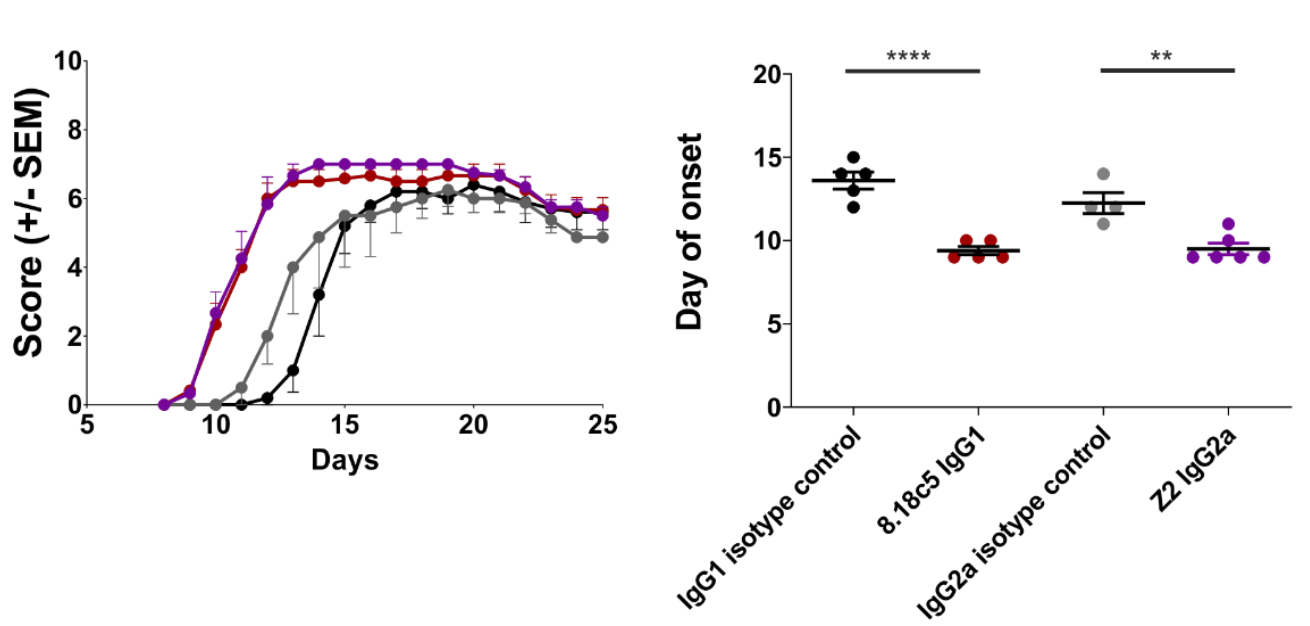

Figure 18. Z2 IgG2a reproduce disease onset acceleration and disease severity effect of 8.18c5 IgG1. C57BI6/J animals were intravenously injected with 8.18c5 IgG1 (red), Z2 IgG2a (purple), IgG1 isotype control (black) or lgG2a isotype control (grey) on d8 after immunization with $25 \mu \mathrm{g}$ of $\mathrm{MOG}_{35-55}$. The clinical course of the disease (left), day of onset (middle) and disease incidence (right) are depicted.

\subsection{18c5 IgG1 re-activate more efficiently infiltrating $T$ cells than other antibody isotypes at the onset of $\mathrm{EAE}$}

Previous results from our department showed that MOG-specific antibodies of the IgG1 isotype accumulate in local APCs within the meninges and activate autoreactive T cells more efficiently than control antibodies ${ }^{87}$. We hypothesized that the observed differences regarding the disease modifying effects among different antibody isotypes could be a consequence of the potential of different antibody isotypes to reactivate MOGspecific T cells within the CNS. As a consequence, these cells would produce higher amounts of inflammatory mediators and would be more efficiently recruited to the CNS. To address this question, a T cell activation reporter mouse line in which the Nur77 protein is coupled to GFP (Nur 77-GFP) was used. Nur77-GFP animals express GFP upon TCR engagement but not by cytokine signalling ${ }^{101}$. GFP expression is temporary, peaking between 12 and 24 hours after TCR stimulation, enabling us to detect T cell activation directly by FACs analysis during this timeframe. T cells isolated from nur77-GFP animals were used for induction of transfer EAE. The donor cells were injected into CD45.1 congenic recipients, and 8.18c5 IgG1, IgG2b and IgE, or their respective isotype controls, were administered i.v. on day 2 post transfer. The animals were sacrificed at disease onset and the percentage of GFP+ T cells was assessed by FACs analysis. Additionally, GFP+ and GFP- cells were sorted to compare cytokine expression levels directly on a per cell basis.

While in the blood no GFP+ T cells were found, activated GFP+ T cells were clearly present in the meninges and spinal cord parenchyma (Figure 19A and 19B). In the meninges, the percentage of GFP positive cells was higher upon administration of $8.18 \mathrm{c} 5 \operatorname{lgG} 1$ compared to $\operatorname{lgG} 1$ isotype control. Administration of $8.18 \mathrm{c5}$ IgG2b led to a non-significant enhancement of activated cells. However, this was not the case upon administration 
of $8.18 \mathrm{c} 5 \mathrm{IgE}$, which induced similar percentages of GFP positive $\mathrm{T}$ cells in the meninges. Interestingly, administration of $8.18 \mathrm{c} 5 \lg \mathrm{g} 2 \mathrm{~b}$ led to higher percentages of activated T cells compared to $8.18 \mathrm{c} 5 \mathrm{IgE}$. In the spinal cord parenchyma, significantly higher numbers of activated CD4+ T cells upon administration of $8.18 \mathrm{c} 5$ IgG1 were observed, while similar results to the meningeal compartment were obtained upon administration of $8.18 \mathrm{c} 5 \operatorname{lgG} 2 \mathrm{~b}$ and IgE. At the cellular level, cytokine expression in GFP positive cells was increased compared to non-activated (GFP-) T cells, but no differences were observed among the different 8.18c5 antibody isotypes and their isotype controls in any analysed tissue. These results indicate that $8.18 \mathrm{c5} \operatorname{lgG} 1$ isotype is able to activate quantitatively higher numbers of infiltrating $T$ cells compared to $8.18 \mathrm{c} 5 \mathrm{IgE}$, but the expression levels of pro-inflammatory cytokines in activated T cells were not enhanced on a per-cell basis among the activated T cells. Regarding IgG2b, the results need to be verified and substantiated in additional experiments. 

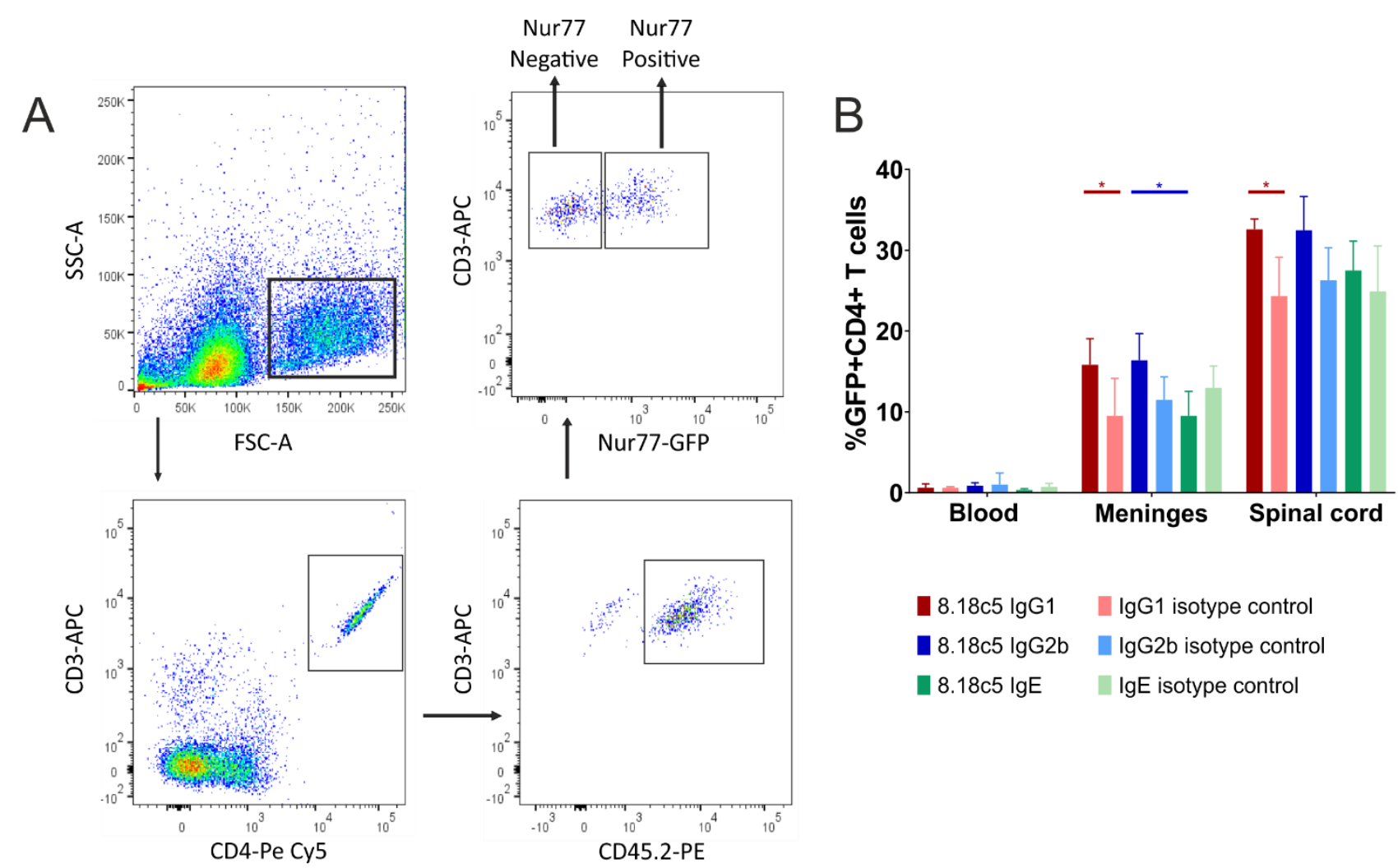

C GFP - Blood GFP +
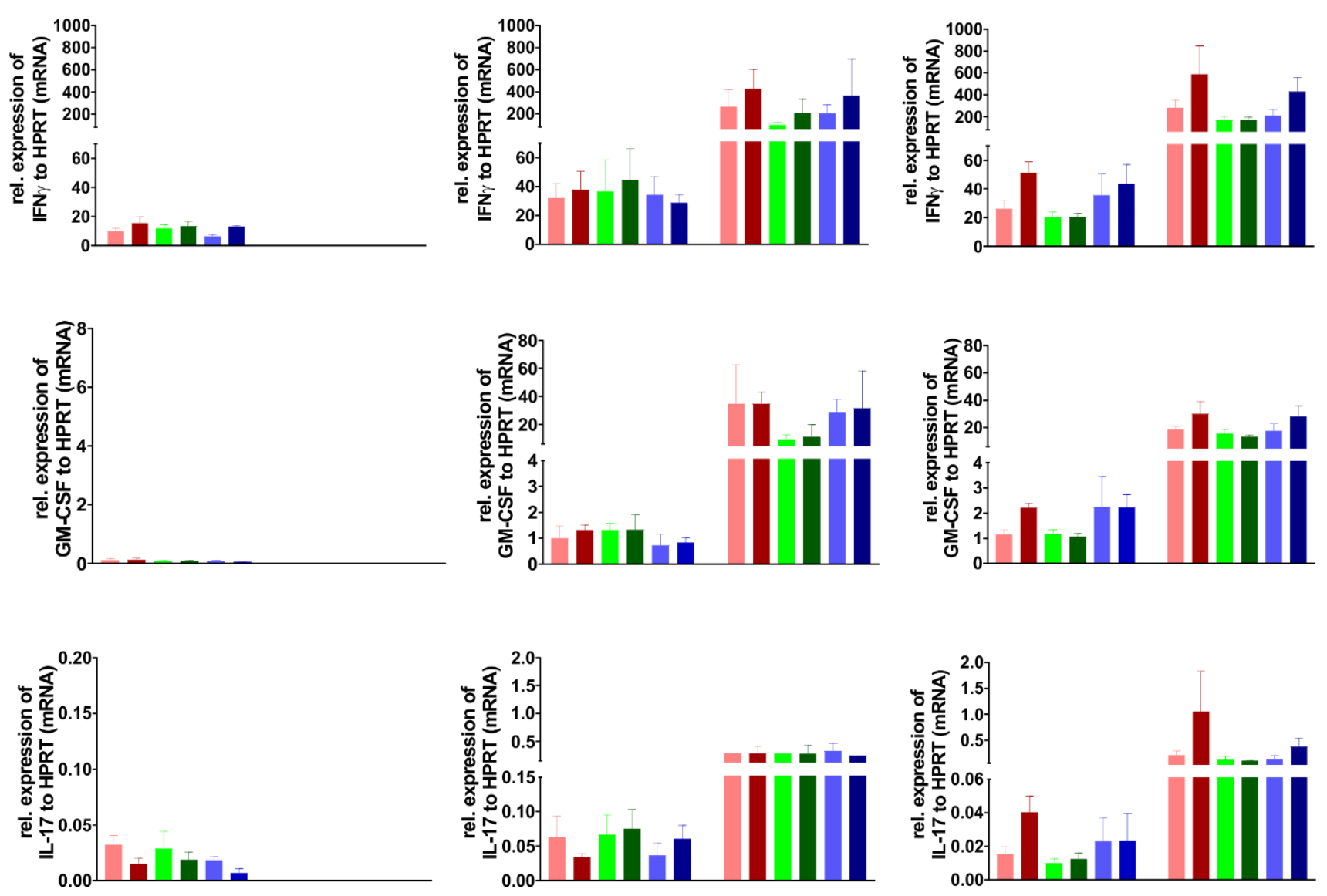
Figure 19. 8.18c5 IgG1 reactivate quantitavely more autoreactive effector T cells than its antibody isotype counterparts at the onset of EAE. EAE was induced by adoptive transfer of encephalitogenic MOG-specific T cells derived from Nur77GFP animals. Antibodies were injected i.v. on d2 post transfer. Upon appearance of the first clinical symptoms, CD4+ T cells were sorted from blood, meninges and spinal cord. A) Gating strategy followed for cell sorting. Nur77-GFP T cells from donor animals were identified by their expression of CD3, CD4 and CD45.2. Activated and non-activated CD4+ T cells were distinguished by their GFP expression as indicated. B) Percentage of GFP+ T cells in blood, meninges and spinal cord parenchyma in $8.18 \mathrm{c} 5$ (IgG1 dark red, $n=5$; IgG2b dark blue, $n=4$; IgE dark green, $n=4$ ) or isotype control (IgG1 isotype control light red, n=5; IgG2b isotype control light blue, $n=4$; IgE isotype control light green, $n=4$ ) treated animals at disea se onset. C) Expression of the pro-inflammatory cytokines IFNY (top), GM-CSF (middle) and IL-17 (bottom) of non-activated (left panel) and activated (right panel) CD4+ T cells determined by qRT-PCR ( $n=2-5$ per case).

\subsection{Antibody isotype determines CNS demyelination and macrophage recruitment to the CNS in EAE}

\subsubsection{Antibody-driven CNS demyelination is dose- and isotype-dependent}

Direct binding of MOG-specific antibodies to myelin can contribute to demyelination through ADCC or activation of the complement cascade ${ }^{38,110}$. Moreover, antibodies opsonizing myelin debris can be recognized by resident glial cells and infiltrating myeloid cells through their Fc receptors, which can trigger further immune responses resulting in CNS tissue destruction. Our previous results using relatively low concentrations of antibodies (50 $\mu \mathrm{g}$ /animal) showed that MOG-specific IgG1 did not cause severe demyelination in preclinical EAE stages independently of CNS inflammation ${ }^{87}$. In contrast, other studies found enhanced demyelination at later EAE stages in 2D2 animals, which contain MOG-specific T cells, upon administration of both Th serum and $150 \mu \mathrm{g}$ of $8.18 \mathrm{c} 5 \operatorname{lgG} 1^{86}$. These data raised the question whether increasing antibody dosing would enhance spinal cord demyelination in WT animals at later time points, and whether the antibody isotype could have an effect on demyelination.

In order to address this question, animals were immunized and administered with increasing doses of $8.18 \mathrm{c} 5$ IgG1 or IgG1 isotype control, and spinal cord demyelination was evaluated by LFB staining in paraffinembedded sections. At the lumbar level, the most affected CNS region by demyelination in our model, 8.18c5 IgG1 antibodies did not enhance spinal cord demyelination at a dose of $50 \mu \mathrm{g}$ compared to IgG1 isotype control (Figure 20A top and 20B). However, i.v. injection of $125 \mu \mathrm{g}$ and $250 \mu \mathrm{g}$ of 8.18c5 IgG1 significantly enhanced spinal cord demyelination, reaching a peak of $18 \%$ demyelinated area of the white matter at the maximum dose. These results indicate that triggering certain antibody effector functions require to overcome a minimum threshold in our EAE model. Administration of increasing concentrations of 8.18c5 IgE did not lead to an enhancement of spinal cord demyelination in a dose-response manner (Figure 20A top and 20B). In contrast, administration of $50 \mu \mathrm{g}$ of $8.18 \mathrm{c} 5 \operatorname{lgG} 2 \mathrm{~b}$ induced $20 \%$ demyelination of the white matter of the spinal cord, a percentage significantly higher than the one induced upon administration of $50 \mu \mathrm{g}$ of $8.18 \mathrm{c} 5 \mathrm{IgG1}$. Even though a dose of $125 \mu \mathrm{g}$ of $8.18 \mathrm{c} 5 \mathrm{lgG} 2 \mathrm{~b}$ did not enhanced spinal cord demyelination compared with $50 \mu \mathrm{g}$ of 8.18c5 IgG2b, animals receiving $250 \mu \mathrm{g}$ of $8.18 \mathrm{c} 5$ IgG2b showed enhanced spinal cord demyelination compared to 
the minimum dose of $8.18 \mathrm{c} 5 \operatorname{lgG} 2 \mathrm{~b}$, reaching a peak of $30 \%$ of the white matter (Figure 20A top and 20B). The latter dose also induced higher spinal cord demyelination compared to the maximum dose of $8.18 \mathrm{c} 5 \operatorname{lgG} 1$. These results indicate the $8.18 \mathrm{c} 5 \operatorname{lgG} 2 \mathrm{~b}$ is more efficient in targeting spinal cord demyelination than $8.18 \mathrm{c5}$ $\operatorname{lgG1}$, and that IgE cannot trigger this process.

Previous studies in Lewis rats showed that Z2 IgG2a has a higher demyelination capacity than 8.18c5 IgG1 antibodies $^{84}$. These observations were based on the administration of high antibody amounts ( $3 \mathrm{mg}$ of antibodies per animal). Therefore, the demyelinating potential of different doses of Z2 IgG2a antibodies in our system was investigated. Compared to IgG2a isotype control, $50 \mu \mathrm{g}$ of Z2 IgG2a did enhance spinal cord demyelination, reaching a peak of $16 \%$ of the white matter (Figure $20 \mathrm{~A}$ top and 20B). These results indicate that also at low doses, Z2 IgG2a antibodies have more demyelinating potential than 8.18c5 IgG1 antibodies. Increasing concentrations of Z2 IgG2a enhanced spinal cord demyelination in a dose-dependent manner, reaching a peak of 30\% demyelination of the white matter at the highest dose (Figure 20A top and 20B). In addition, the $Z 2$ antibody isotype seemed to be crucial for demyelination, since no dose-dependency pattern was observed when animals received increasing doses of Z2 IgE and IgA (Figure 20A top and 20B). In line with the results obtained with the 8.18c5 antibodies, an enhancement of spinal cord demyelination could be observed only in the case when Z2 antibodies of the IgG isotype were administered. Statistical comparisons are summarized in Table 5.

The observed patterns for all these antibody isotypes were further confirmed at the thoracic level, although the animals showed lower spinal cord demyelination percentages in this region. At the cervical level, $250 \mu \mathrm{g}$ of Z2 IgG2a still enhanced spinal cord demyelination compared to IgG2a isotype control. Other 8.18c5 and Z2 antibody isotypes did not induce additional demyelination in this region of the spinal cord (Figure 20A).

Confirming our previous results regarding the acceleration effect of MOG-specific autoantibodies, when assessing the clinical course of the disease in these experiments, disease acceleration was only observed upon administration of different IgG antibody isotypes, but not by IgE or IgAs (Figure 21). This acceleration effect of IgGs was independent of the dose administered. Higher concentrations of IgE and Z2 IgA were not able to induce an earlier disease onset, providing further support to the idea that disease acceleration is an IgG-driven phenomenon (Figure 21). 
A Lumbar SC

Thoracic SC

Cervical SC
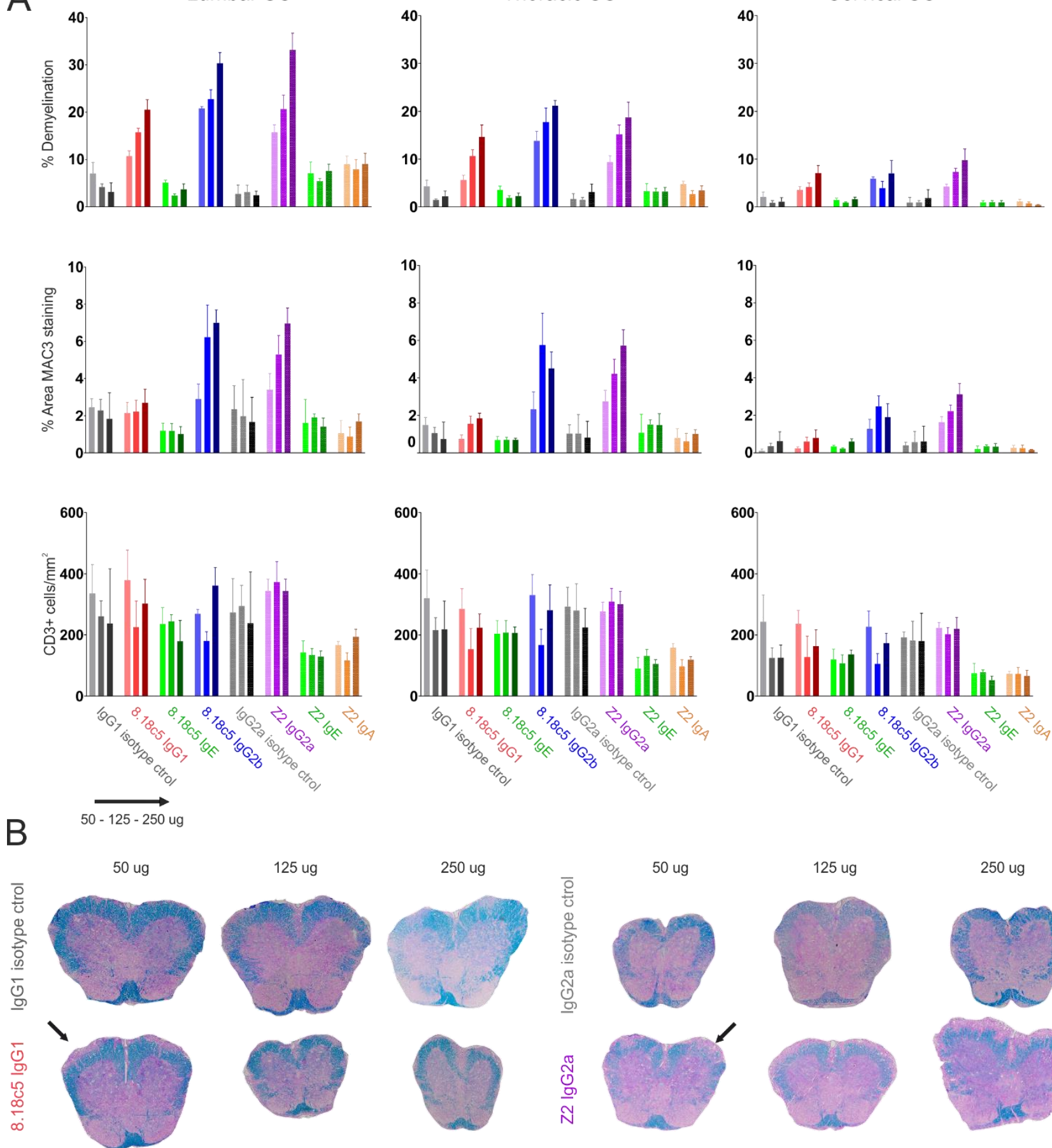

250 ug
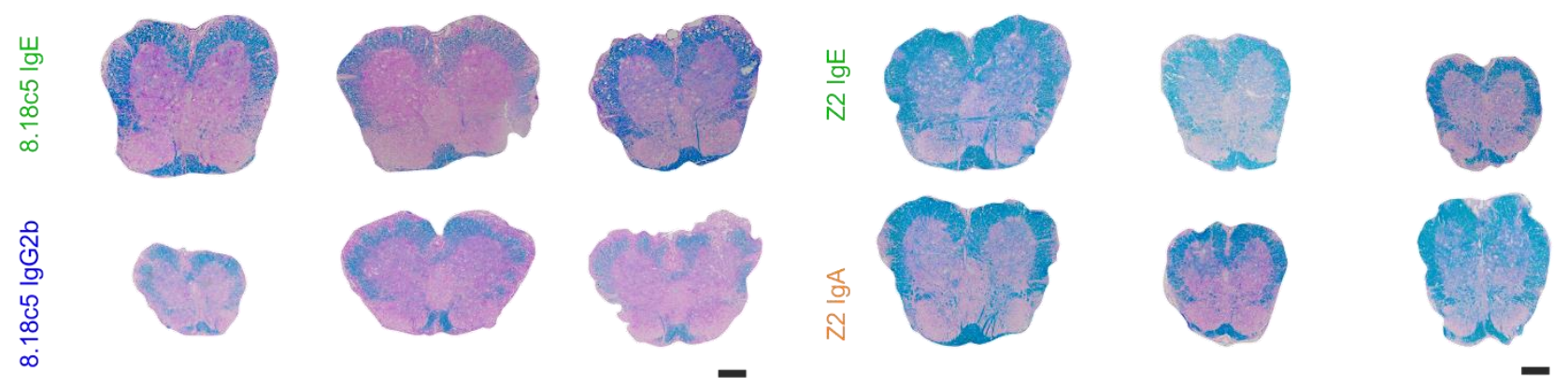
Figure 20. IgGs enhance spinal cord demyelination more efficiently than other antibody isotypes. C57BI6/J were immunized with $50 \mu \mathrm{g}$ of $\mathrm{MOG}_{35-55}$ and injected intravenously on d8 with 50, 125 or $250 \mu \mathrm{g}$ of the indicated $8.18 \mathrm{c} 5, \mathrm{Z2}$ antibodies, or isotype control. Animals were sacrificed at the peak of the disease (Scores 6-7) and spinal cord sections were stained with LFB, anti-MAC3 or anti-CD3 antibodies. A) Top: quantification of the spinal cord demyelination percentage at lumbar (left), thoracic (middle) and cervical (right) regions. Middle: quantification of the percentage of area occupied by the macrophage marker MAC-3 in the spinal cord at lumbar (left), thoracic (middle) and cervical (right) regions. Bottom: quantification of CD3 positive T cell infiltrates in the spinal cord at at lumbar (left), thoracic (middle) and cervical (right) regions. Groups of 3 columns in every experimental case show increasing antibody dosing: $50 \mu \mathrm{g}$ (first column), $125 \mu \mathrm{g}$ (second column), and $250 \mu \mathrm{g}$ (third column). B) Representative LFB-stained spinal cord sections at the lumbar region are depicted. Left panel show 8.18c5 antibodies or isotype control examples. Right panel show Z2 antibodies or isotype control examples. Arrows point at an example of demyelinating lesion considered for the analysis of the LFB staining. Scale bar: $200 \mu \mathrm{m}$.

$8.18 \mathrm{c} 5$ antibodies

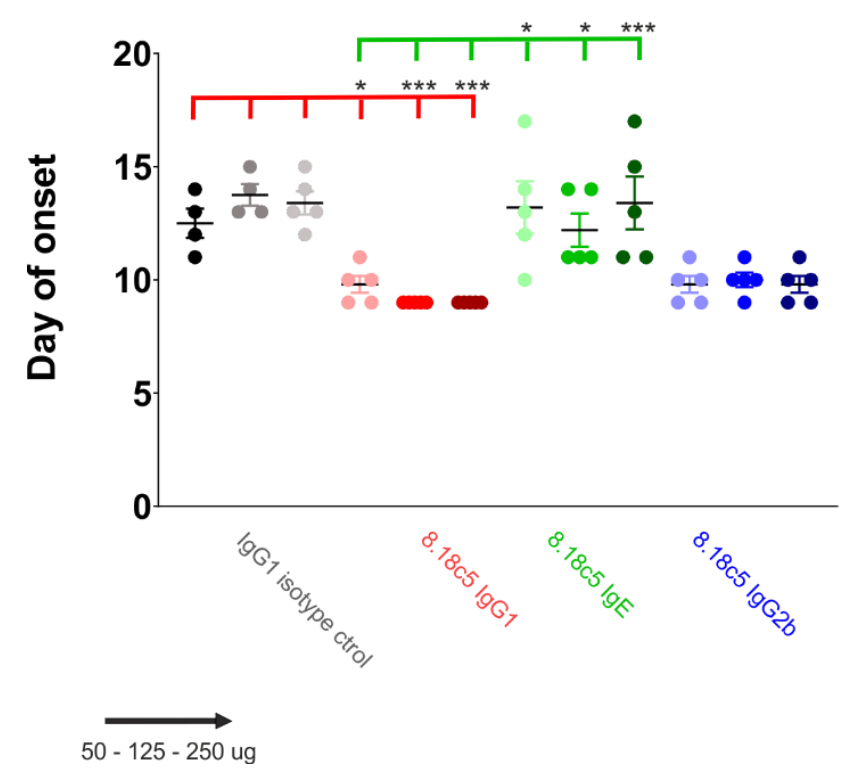

Z2 antibodies

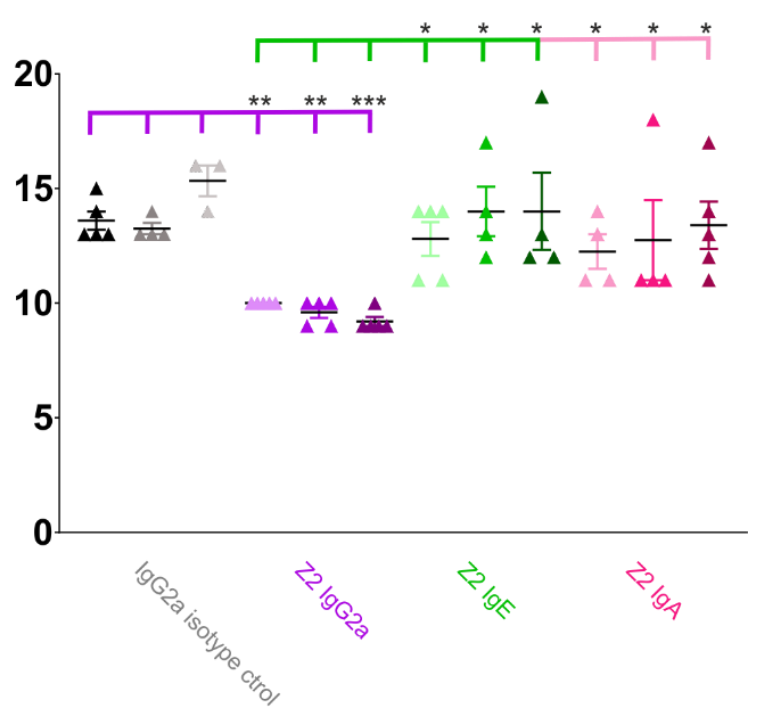

Figure 21. IgGs accelerate disease onset more efficiently than other isotypes independently of the administered dose. $\mathrm{C} 57 \mathrm{Bl} / \mathrm{J}$ were immunized with $50 \mu \mathrm{g}$ of $\mathrm{MOG}_{35-55}$ and injected intravenously on d8 with 50, 125 or $250 \mu \mathrm{g}$ of the indicated $8.18 \mathrm{c} 5, \mathrm{Z2}$ antibodies, or isotype control. Clinical score was evaluated daily and day of disease onset is depicted in the figure. IgG1 isotype control 50-125-250 $\mu$ g (black, n=4-5), 8.18c5 IgG1 50-125-250 $\mu \mathrm{g}$ (red, n=5-6), 8.18c5 IgE 50-125-250 $\mu \mathrm{g}$ (green, n=4-5), 8.18c5 IgG2b 50-125-250 $\mu$ g (blue, n=5-6), IgG2a isotype control 50-125-250 $\mu$ g (black, n=3-5), Z2 IgG2a

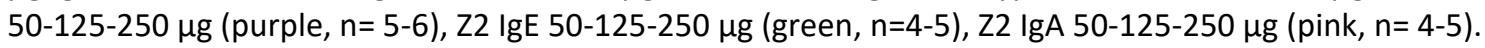

\subsubsection{Macrophage and $T$ cell infiltration is not different upon administration of low doses of different $8.18 \mathrm{c} 5$ or $\mathbf{Z 2}$ antibody isotypes}

In the EAE model, infiltrating T-cells and macrophages, and their inflammatory mediators, mediate CNS inflammatory damage leading to the CNS demyelination ${ }^{11,111}$. Consequently, we evaluated macrophage and T cell infiltration in the CNS upon administration of different antibody isotypes following the experimental design described in the previous chapter. Macrophage infiltration was evaluated by quantification of the percentage of the area of the white matter occupied by MAC-3 positive cells in spinal cord sections. 
At the lumbar spinal cord, 8.18c5 IgG1 did not enhanced the percentage of the area occupied by MAC-3 positive cells compared to IgG1 isotype control at any administered dose (Figure 20A, middle part). Similar results were obtained upon administration of $8.18 \mathrm{c} 5 \mathrm{IgE}$. Although administration of $50 \mu \mathrm{g}$ of $8.18 \mathrm{c} 5 \operatorname{lgG} 2 \mathrm{~b}$ did not enhanced spinal cord MAC-3 staining, the highest dose promoted an enhancement of MAC-3 positive cells compared to the same dose of $8.18 \mathrm{c} 5 \operatorname{IgG1}$ (one way ANOVA, $p=0.0005$ ), reaching a peak of approximately $7 \%$ occupied area of the white matter.

Administration of either $50 \mu \mathrm{g}$ or $125 \mu \mathrm{g}$ of Z2 IgG2a did not lead to an increased percentage of MAC-3 positive cells within the CNS tissue (Figure 20A). However, i.v. injection of $250 \mu \mathrm{g}$ of Z2 IgG2a enhanced the percentage of MAC-3 positive cells compared to IgG2a isotype control, reaching a peak of 7\%. Additionally, administration of Z2 IgE and IgA did not lead to enhanced MAC-3 staining at any administered dose. Since increased percentages of MAC-3 positive cells were only observed upon administration of high amounts of $8.18 \mathrm{c} 5 \operatorname{lgG} 2 \mathrm{~b}$ and $\mathrm{Z2}$ IgG2a, while low doses of these antibodies already enhanced spinal cord demyelination, these results indicate that enhanced demyelination of the spinal cord is not strongly correlated to higher numbers of macrophages present within the CNS.

Infiltrating T cells numbers and macrophage recruitment to the CNS are closely related in the EAE model ${ }^{112}$. Since $T$ cell recruitment could be affected by the administration of MOG antibodies varying in their isotype, $T$ cell infiltration was assessed by quantification of spinal cord sections stained with antibodies specific for CD3. No differences were found in T cell infiltration at any dose upon administration of 8.18c5 isotypes (Figure 20A, lower part). Upon administration of Z2 IgG2a similar results were obtained, showing that Z2 isotypes did not induce the recruitment of higher numbers of CD3 positive T cells at any administered dose (Figure 20A). These results indicate that different antibody isotypes recruit similar numbers of T cells to the CNS at the peak of the disease. Nevertheless, the presence of higher numbers of MAC-3 positive cells points at a potential role for macrophages mediating these differences in CNS demyelination in an isotype-dependent manner. 
Table 5. Percentage of demyelination statistical analysis at the lumbar spinal cord; experiment depicted in figure 14. One-way ANOVA of 8.18c5 and Z2 antibody isotypes or isotype control, independent from each other, is depicted. The upper part show dose comparison within the same antibody. The lower part show isotypes comparison within the same dosage.

Intra-group comparisons

\subsection{8c5 isotypes}

Statistical significance

\section{IgG1 isotype control}

$50 \mu \mathrm{g}$ vs $125 \mu \mathrm{g}$

$50 \mu \mathrm{g}$ vs $250 \mu \mathrm{g}$

$125 \mu \mathrm{g}$ vs $250 \mu \mathrm{g}$

8.18c5 IgG1

$50 \mu \mathrm{g}$ vs $125 \mu \mathrm{g}$

$50 \mu \mathrm{g}$ vs $250 \mu \mathrm{g}$

$125 \mu \mathrm{g}$ vs $250 \mu \mathrm{g}$

$8.18 \mathrm{c} 5 \lg \mathrm{E}$

$50 \mu \mathrm{g}$ vs $125 \mu \mathrm{g}$

$50 \mu \mathrm{g}$ vs $250 \mu \mathrm{g}$

$125 \mu \mathrm{g}$ vs $250 \mu \mathrm{g}$

$8.18 \mathrm{c} 5 \lg \mathrm{g} 2 \mathrm{~b}$

$50 \mu \mathrm{g}$ vs $125 \mu \mathrm{g}$

$50 \mu \mathrm{g}$ vs $250 \mu \mathrm{g}$

$125 \mu \mathrm{g}$ vs $250 \mu \mathrm{g}$

ns
ns
ns
ns
$* * *$
ns

ns

ns

ns

ns

ns

*

\section{$\mathrm{Z2}$ isotypes}

\begin{tabular}{|c|c|}
\hline \multicolumn{2}{|c|}{ IgG2a isotype control } \\
\hline $50 \mu \mathrm{g}$ vs $125 \mu \mathrm{g}$ & ns \\
\hline $50 \mu \mathrm{g}$ vs $250 \mu \mathrm{g}$ & ns \\
\hline $125 \mu \mathrm{g}$ vs $250 \mu \mathrm{g}$ & ns \\
\hline \multicolumn{2}{|l|}{$\mathrm{Z2} \operatorname{lgG} 2 \mathrm{a}$} \\
\hline $50 \mu \mathrm{g}$ vs $125 \mu \mathrm{g}$ & ns \\
\hline $50 \mu \mathrm{g}$ vs $250 \mu \mathrm{g}$ & $* * * *$ \\
\hline $125 \mu \mathrm{g}$ vs $250 \mu \mathrm{g}$ & $* *$ \\
\hline \multicolumn{2}{|l|}{$\mathrm{Z} 2 \mathrm{lgE}$} \\
\hline $50 \mu \mathrm{g}$ vs $125 \mu \mathrm{g}$ & ns \\
\hline $50 \mu \mathrm{g}$ vs $250 \mu \mathrm{g}$ & ns \\
\hline $125 \mu \mathrm{g}$ vs $250 \mu \mathrm{g}$ & ns \\
\hline \multicolumn{2}{|l|}{$\mathrm{Z2} \lg \mathrm{A}$} \\
\hline $50 \mu \mathrm{g}$ vs $125 \mu \mathrm{g}$ & ns \\
\hline $50 \mu \mathrm{g}$ vs $250 \mu \mathrm{g}$ & ns \\
\hline $125 \mu \mathrm{g}$ vs $250 \mu \mathrm{g}$ & ns \\
\hline
\end{tabular}

Inter-group comparisons

\subsection{8c5 isotypes}

\section{$\mathrm{Z2}$ isotypes}

Statistical significance

8.18c5 IgG1 vs

IgG1 isotype control

$\begin{array}{cc}50 \mu \mathrm{g} & \mathrm{ns} \\ 125 \mu \mathrm{g} & * * * \\ 250 \mu \mathrm{g} & * * * *\end{array}$

8.18c5 IgG1 vs IgE

$50 \mu \mathrm{g}$

$125 \mu \mathrm{g}$

$250 \mu \mathrm{g}$

$8.18 c 5 \lg G 1$ vs $\lg G 2 b$

$50 \mu \mathrm{g}$

$125 \mu \mathrm{g}$

$250 \mu \mathrm{g}$

$8.18 \mathrm{c} 5 \lg$ v v $\lg \mathrm{g} 2 \mathrm{~b}$

$50 \mu \mathrm{g}$

$125 \mu \mathrm{g}$

$250 \mu \mathrm{g}$

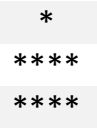

$* *$

$*$

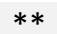

$* * * *$

$* * * *$

$* * * *$
$Z 2$ IgG2a vs
IgG2a isotype co

$$
\begin{aligned}
& 50 \mu \mathrm{g} \\
& 125 \mu \mathrm{g} \\
& 250 \mu \mathrm{g}
\end{aligned}
$$

Z2 IgG2a vs IgE

$50 \mu \mathrm{g}$

$125 \mu \mathrm{g}$

$250 \mu \mathrm{g}$

Statistical significance

Z2 IgG2a vs IgA

$50 \mu \mathrm{g}$
$125 \mu \mathrm{g}$
$250 \mu \mathrm{g}$
$\mathrm{IgE} \mathrm{vs} \mathrm{IgA}$
$50 \mu \mathrm{g}$
$125 \mu \mathrm{g}$
$250 \mu \mathrm{g}$

$50 \mu \mathrm{g}$

$125 \mu \mathrm{g}$

2 IgE vs IgA

$50 \mu \mathrm{g}$

$250 \mu \mathrm{g}$ 


\subsubsection{Intrathecal administration of low doses of 8.18c5 antibody isotypes also enhance CNS demyelination}

Since we observed enhanced demyelination upon i.v. injection of high doses of 8.18c5 IgG1, as well as at every administered dose of $8.18 \mathrm{c} 5 \operatorname{lgG} 2 \mathrm{~b}$, the effect of $8.18 \mathrm{c} 5$ isotypes when administered directly into the CNS at two different doses $(10 \mu \mathrm{g}$ and $50 \mu \mathrm{g})$ was investigated. In this experimental setup, animals were injected with either IgG1 isotype control or 8.18c5 antibody isotypes i.t. before disease onset, and sacrificed at the peak of the disease. 8.18c5 IgG1 did not enhanced demyelination compared to isotype control when a dose of $10 \mu \mathrm{g}$ was i.t. administered (Figure 22A and 22B). However, $50 \mu \mathrm{g}$ of 8.18c5 $\operatorname{lgG} 1$ did enhanced demyelination compared to IgG1 isotype control. These results go in line with our observations in the i.v. administration context, and support the hypotheses that higher concentrations of $8.18 \mathrm{c} 5 \operatorname{lgG} 1$ are required for this antibody isotype in order to exert demyelination efficiently. 8.18c5 IgE did not show increasing demyelination capacity upon increasing dosage (Figure 22A and 22B). Administration of $10 \mu \mathrm{g}$ of $8.18 \mathrm{c} 5 \operatorname{lgG} 2 \mathrm{~b}$ enhanced spinal cord demyelination compared to the same dose of $8.18 \mathrm{c} 5 \operatorname{lgG1}$, reproducing the effect observed after i.v. administration of MOG-specific IgG2b antibodies. However, the demyelination percentage after administration of $50 \mu \mathrm{g}$ of $8.18 \mathrm{c} 5 \mathrm{lgG} 2 \mathrm{~b}$ was not significantly different from the one obtained with the lower dose and was also similar to the same dose of $8.18 \mathrm{c} 5 \lg G 1$. Taken together these results further support the idea that both antibody dosage and antibody isotype determines the outcome of pathological effector mechanisms within the CNS, independently from peripheral mechanisms. 


\section{A}

IgG1 isotype control 10 ug

8.18c5 lgG1 $10 \mathrm{ug}$

IgG1 isotype control $50 \mathrm{ug}$

- 8.18c5 IgG1 $50 \mathrm{ug}$

8.18c5 lgE 10 ug

8.18c5 IgG2b 10 ug

$8.18 \mathrm{c} 5 \lg \mathrm{E} 50 \mathrm{ug}$

$8.18 c 5$ IgG2b 50 ug

\section{Lumbar SC}

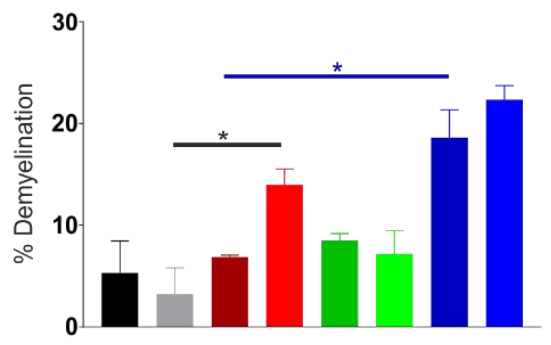

Thoracic SC

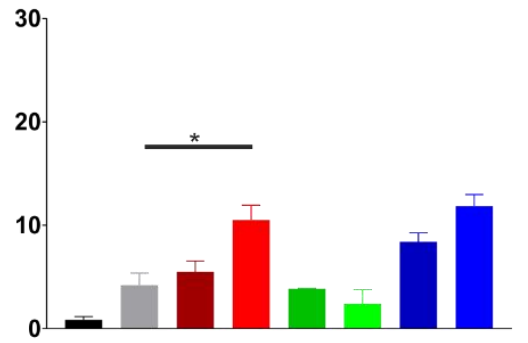

Cervical SC

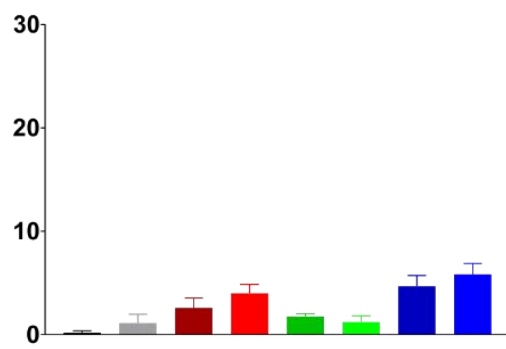

B

IgG1 isotype ctrl $10 \mathrm{ug}$

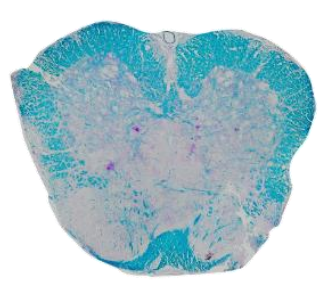

8.18c5 lgE $10 \mathrm{ug}$

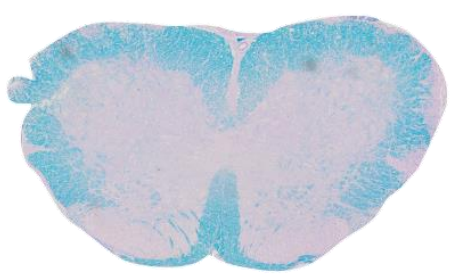

IgG1 isotype ctrl 50 ug

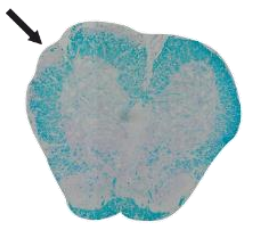

$8.18 c 5 \lg E 50$ ug

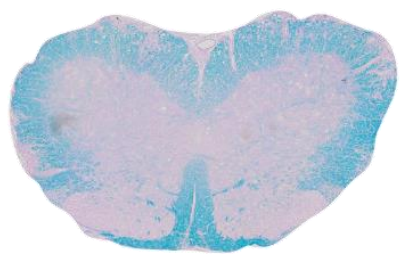

8.18c5 IgG1 $10 \mathrm{ug}$

$8.18 c 5 \lg G 150 u g$

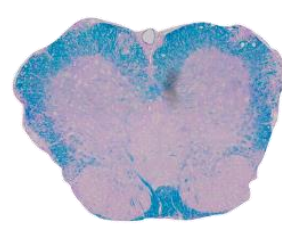

$8.18 \mathrm{c} 5 \lg G 2 \mathrm{~b} 10 \mathrm{ug}$

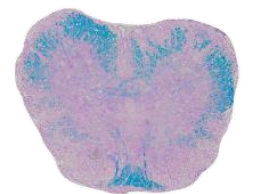

$8.18 c 5 \lg G 2 b 50$ ug
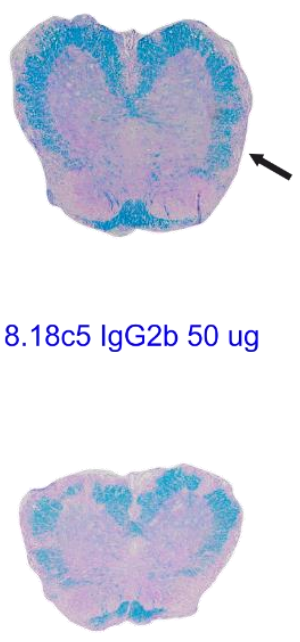

Figure 22. 8.18c5 IgGs enhance spinal cord demyelination when administered directly into the CNS. C57BI6/J were immunized with $50 \mu \mathrm{g}$ of $\mathrm{MOG}_{35-55}$ and injected intrathecally on d8 with 10 or $50 \mu \mathrm{g}$. Animals were sacrificed at the peak of the disease (Scores 6-7) and spinal cord sections were stained with LFB. A) Quantification of spinal cord demyelination percentage at the lumbar (left), thoracic (middle) and cervical (right) spinal cord regions. B) Representative LFB-stained spinal cord sections at the lumbar region are depicted. Arrows point at an example of demyelinating lesion considered for the analysis of the LFB staining. Scale bar: $200 \mu \mathrm{m}$. 


\subsubsection{Administration of $8.18 \mathrm{c} 5$ antibody isotypes at the peak of EAE does not lead to enhanced demyelination}

In our model, crucial immune processes such as priming of cells in the draining lymph nodes as well as T-cell reactivation in the meninges by local APCs have already occurred at the peak of the disease. Since we observed variations in the demyelination potential of different antibody isotypes when administered both i.v. or i.t. at early time points, which were not completely correlated to infiltration of macrophages and T cells, we investigated whether enhanced demyelination could also be observed upon antibody administration at later time points. In order to address this question, $\mathrm{C} 57 \mathrm{BI} / \mathrm{J}$ animals were immunized with $\mathrm{MOG}_{35-55}$ and injected i.t. with $10 \mu \mathrm{g}$ of different $8.18 \mathrm{c} 5$ antibody isotypes or $\lg \mathrm{G} 1$ isotype control at the peak of the disease. 24 hours after i.t. injections we did not find significant differences in demyelination between 8.18c5 IgG1 and IgG1 isotype control (Figure 23A and 23B, upper part). Moreover, no differences in spinal cord demyelination were found upon administration of 8.18c5 IgE and IgG2b compared to 8.18c5 IgG1. Quantification of MAC-3 staining and number of infiltrating CD3 positive $T$ cells did also show no difference among the different $8.18 \mathrm{c} 5$ antibodies. Since the chosen time point could be too early to observe any effects, these parameters were addressed 3 days after antibody i.t. injection at the peak of EAE. Similar to the previous experiment, demyelination was not significantly enhanced three days after administration of antibodies, although a clear tendency towards an enhanced demyelination could be observed upon administration of $8.18 \mathrm{c} 5$ IgG2b (Figure 23A and 23B, lower part). Moreover, no differences were found in the area covered by MAC-3 positive cells or numbers of infiltrating CD3 positive cells. These results indicate that in spite of the slight enhancement that can be observed upon administration of $8.18 \mathrm{c} 5 \lg 2 \mathrm{~b}, 8.18 \mathrm{c} 5$ antibodies exert their pathogenic functions efficiently when administered before the entrance of autoreactive cells to the CNS. 
A
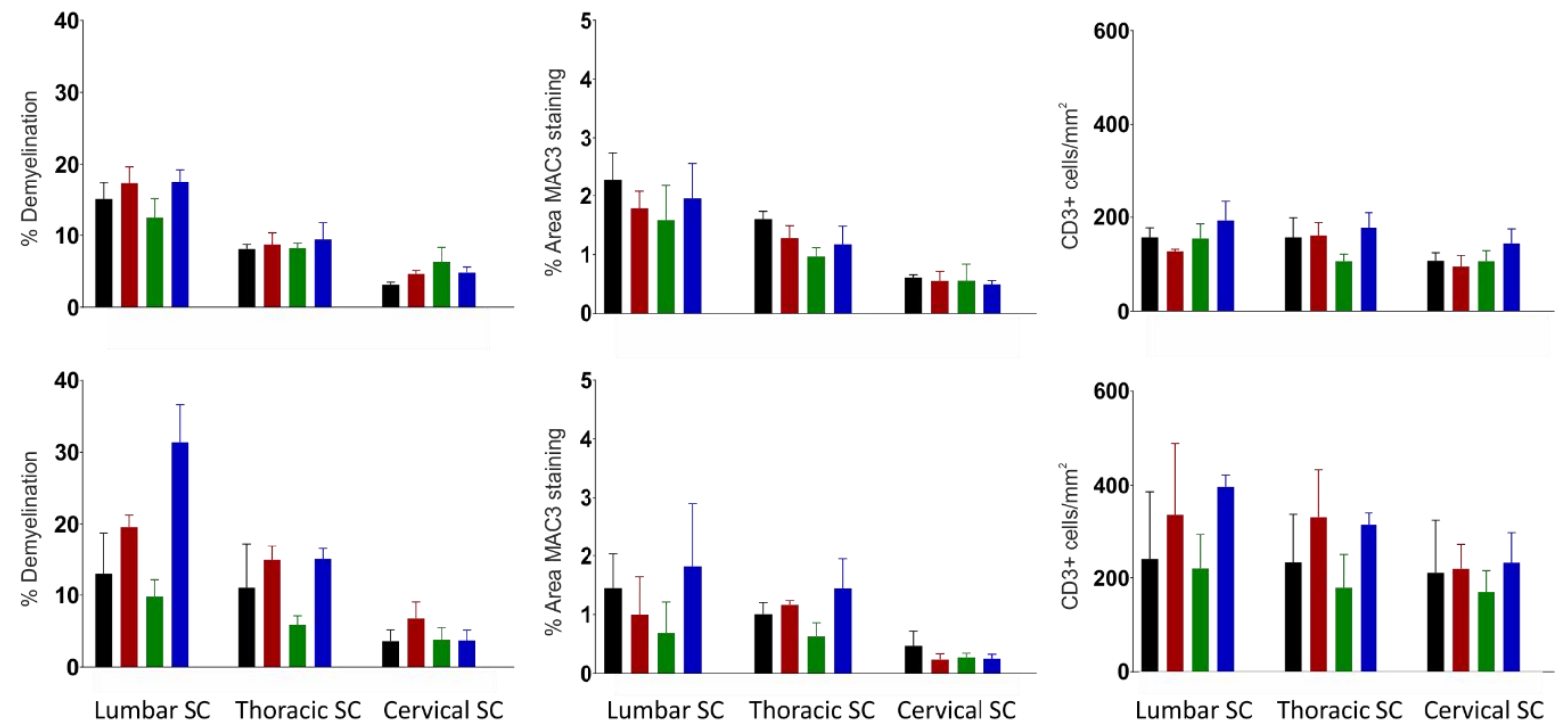

B

IgG1 isotype control

$8.18 \mathrm{c} 5 \lg \mathrm{g} 1$

$8.18 c 5 \lg E$
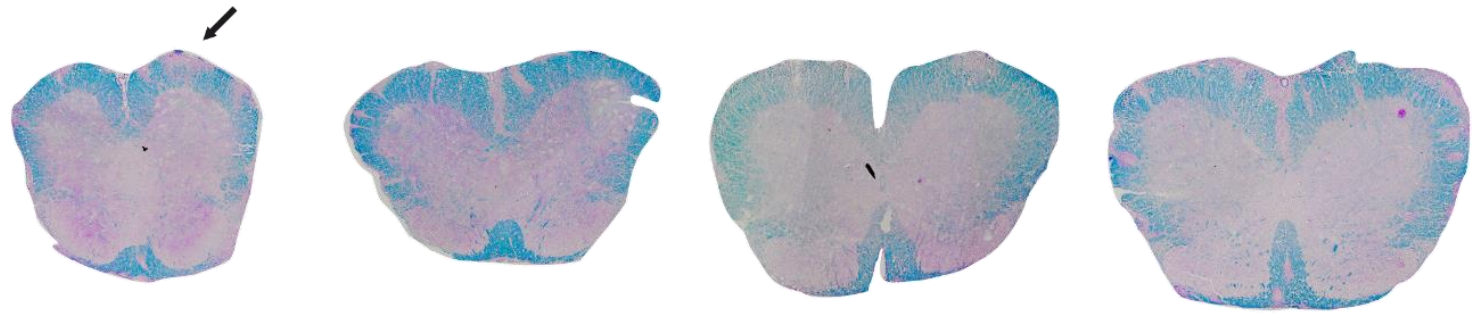

72 hours
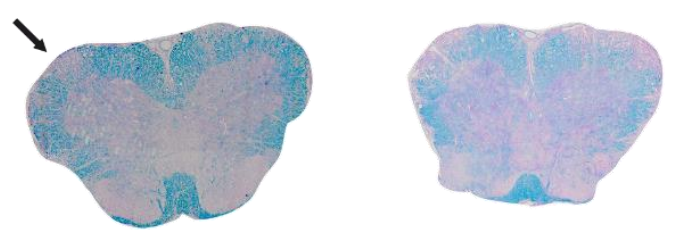

$8.18 c 5 \lg G 2 b$

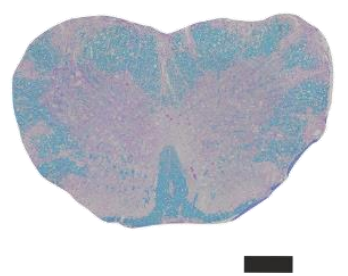

Figure 23. Intrathecal administration of 8.18c5 antibodies or isotype control at the peak of EAE does not lead to enhanced spinal cord demyelination. C57Bl6/J were immunized with $50 \mu \mathrm{g}$ of $\mathrm{MOG}_{35-55}$ and injected intrathecally at the peak of the disease (Scores 6-7) with $10 \mu \mathrm{g}$ of 8.18c5 IgG1, IgE, IgG2b or IgG1 isotype control. Animals were sacrified 24 hours later (upper panels) or 72 hours later (lower panels). A) Quantification of spinal cord demyelination percentage at the lumbar (left), thoracic (middle) and cervical (right) regions. B) Representative LFB-stained spinal cord sections at the lumbar region are depicted. Arrows point at an example of demyelinating lesion considered for the analysis of the LFB staining. Scale bar: $200 \mu \mathrm{m}$. 


\subsection{Antibody-dependent effector mechanisms within the CNS are dependent on FC receptors expression}

\subsubsection{MOG-specific IgG1 autoantibodies enhance CNS demyelination in Fcy receptor knock out animals but do not accelerate EAE onset}

Autoantibodies can target myelin structures upon generation of immunocomplexes and their subsequent recognition by cells expressing Fc receptors, which would ultimately exert CNS damage through ADCC. We reasoned that isotype-dependent demyelination patterns might be modified upon genetic removal of different Fc receptors. To this end, several KO models for different Fc receptors were tested. The animal models used in this experimental setup were the KO for the Fc neonatal receptor (FcRn), which is implicated in recycling and intracellular processing of IgGs and consequently increasing the half-life time of immunoglobulins ${ }^{113}$; KO animals for the common $\gamma$-chain + FcyRIIB, where activating FcyRs I, III and IV, and the inhibitory FcyRIIB are affected; KO animals for the alpha chains of FcyRs I and III; and KO animals for the alpha chains of FcyRI, IIB, III and IV (FcyRI-IV). The animals were immunized with $50 \mu \mathrm{g}$ of $\mathrm{MOG}_{35-55}$ plus PTX, and administered i.v. with $50 \mu \mathrm{g} 8.18 \mathrm{c} 5 \lg G 1$ or $\lg G 1$ isotype control shortly before the clinical onset of the disease. The acceleration of disease onset observed upon administration of 8.18c5 IgG1 in WT mice was reproduced in all the KO models, with the exception of the FcyRI-IV KO animals (Figure 24A), supporting previous observations from our department ${ }^{109}$. These latter animals showed no differences in the day of onset of EAE compared to isotype control, while this effect was still present in both WT animals and mice in which only parts of the FcyR repertoire were deleted. Demyelination was not enhanced upon administration of 8.18c5 IgG1 in WT animals (Figure 24B and 24C), similar to our previous observations. FcRn KO, FcR common- $\gamma+$ FcyRIIB KO and FcyRI+III KO animals did also not show enhanced demyelination when administered with 8.18c5 IgG1. However, a significant enhancement of demyelination was found in FcyRI-IV KO animals (Figure 24B and 24C). Intriguingly, these animals showed already enhanced demyelination compared to WT animals when treated with the isotype control antibody, indicating that CNS demyelination is generally enhanced upon removal of the Fcy receptor alpha chains. Upon treatment with MOG-specific antibodies, FcyRI-IV KO mice showed a highly significant increase in demyelination (Figure 24B and 24C), suggesting that 8.18c5 IgG1 antibodies are able to exert higher amounts of demyelination in the absence of Fcy receptors.

Importantly, the abrogation of disease acceleration by 8.18c5 IgG1 in FcyRI-IV KO animals together with the enhanced spinal cord demyelination suggest that disease acceleration and demyelination are driven by different mechanisms. These results also suggest that the role of antibodies in specific effector mechanisms can be modified through the ablation of certain molecular cues, resulting in different types of effector mechanism in the absence of the preferred system (for example, Fc receptor binding or complement cascade activation). 
Finally, quantification of MAC3 positive cells and CD3 positive cells in these samples did not show any significant difference among the $\mathrm{KO}$ animals tested, indicating the macrophage and $\mathrm{T}$ cell recruitment are not in principle responsible for the observed differences regarding EAE disease onset and enhanced demyelination in FcyRI-IV KO animals (Figure 24B). 

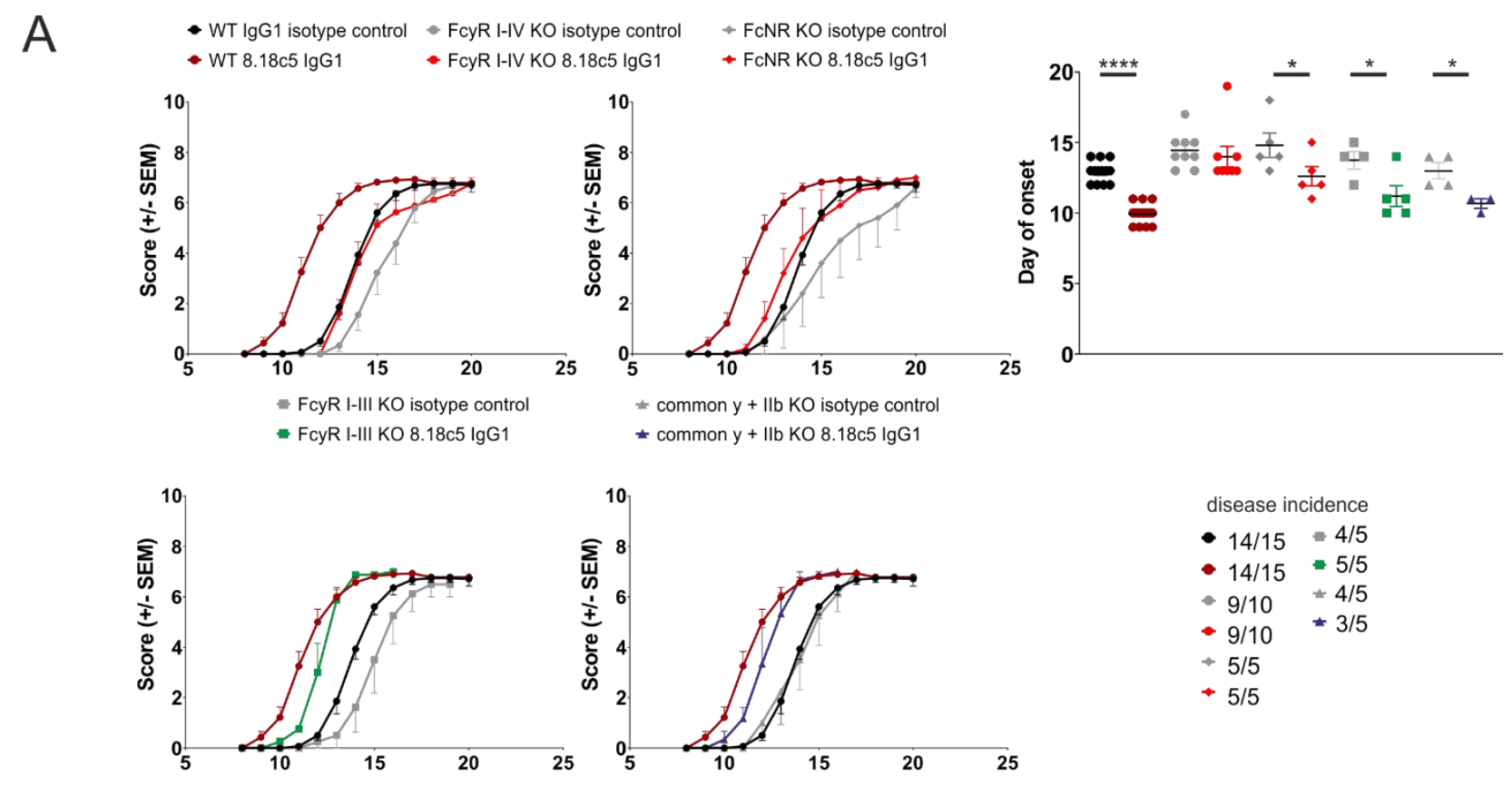

disease incidence

- $14 / 15 \quad 4 / 5$

- $14 / 15=5 / 5$

- $9 / 10 \neq 4 / 5$

- $9 / 10 \neq 3 / 5$

$+5 / 5$

$+5 / 5$

$\begin{array}{ll}\text { WT isotype control } & \text { FcNR KO isotype contro } \\ \text { WT 8.18c5 IgG1 } & \text { FcNR KO 8.18c5 IgG1 }\end{array}$ 40
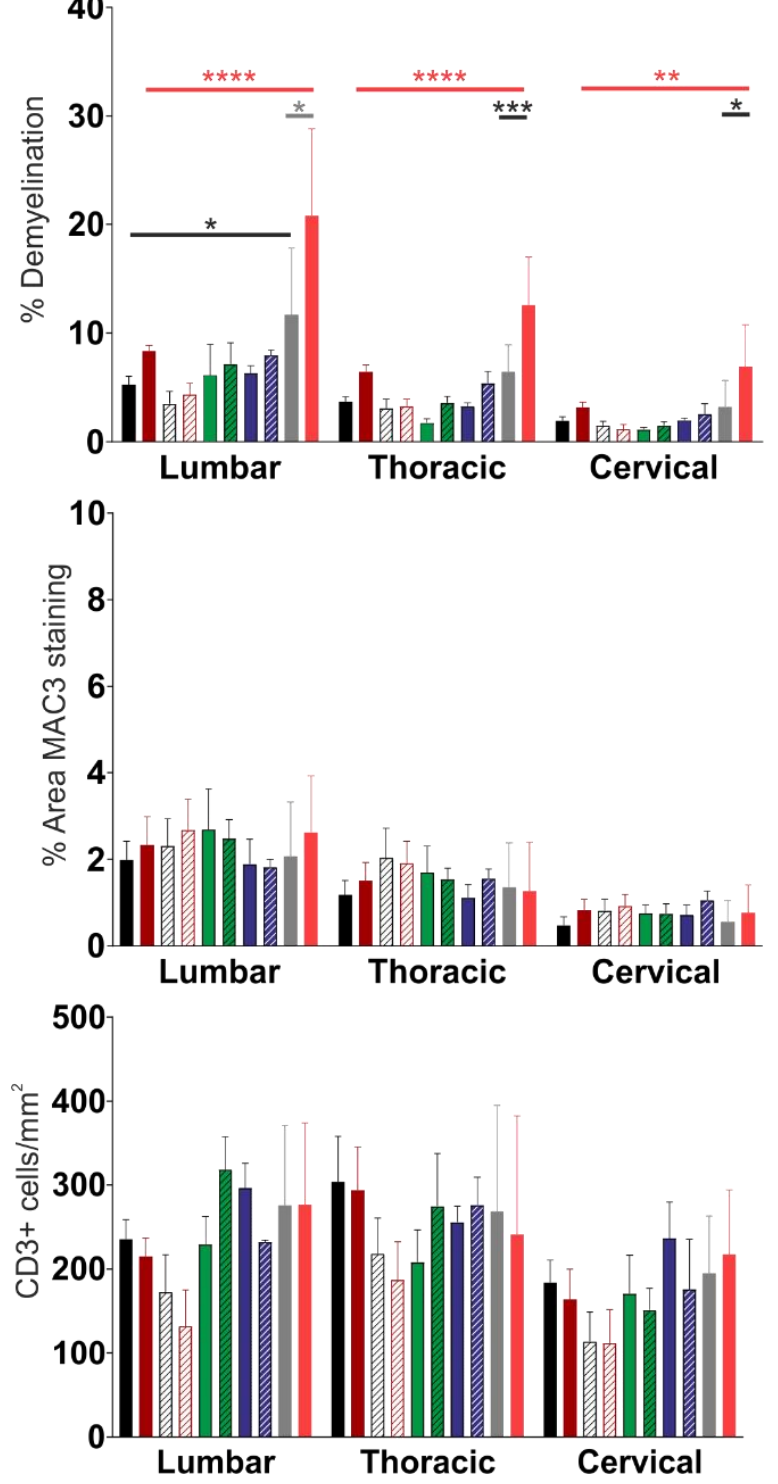
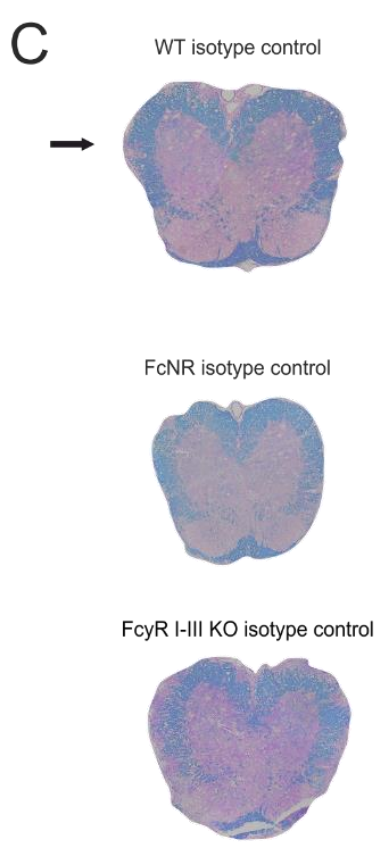

Common y + IIB isotype control

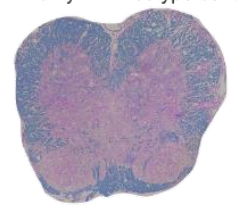

FcyR I-IV KO isotype control

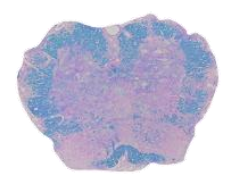

FcyR I-IV KO isotype control - FcyR I-IV KO 8.18c5 IgG1

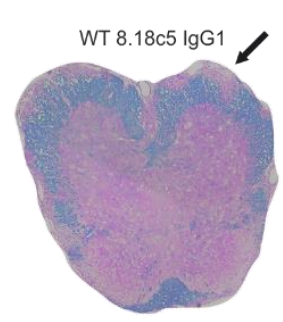

FcNR $8.18 \mathrm{c} 5 \lg$ G1

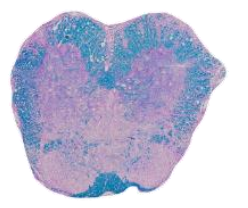

FcyR I-III KO 8.18c5 IgG1

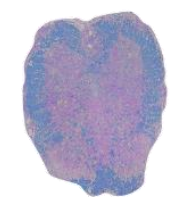

Common y + IIB 8.18c5 lgG1

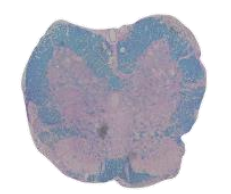

FcyR I-IV KO 8.18c5 IgG1

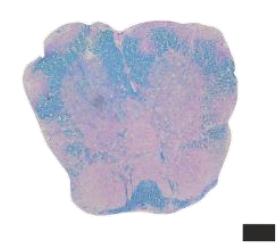


Figure 24. 8.18c5 IgG1 enhance spinal cord demyelination more efficiently upon knock-out of the activating Fc receptors alpha chain. C57BI6/J WT, FcyR I-III-IV KO, FCNR KO, FcyR I-III and common y chain KO animals were immunized with $50 \mu \mathrm{g}$ of $\mathrm{MOG}_{35-55}$ and intravenously injected with $50 \mu \mathrm{g}$ of $8.18 \mathrm{c} 5 \operatorname{lgG} 1$ or IgG1 isotype control. A) EAE clinical course of WT animals vs the corresponding KO model (left), day of onset (right upper), and disease incidence (right lower). EAE curves represent two independent experiments for WT and FcyR I-III-IV KO, and one experiment for the other KO models. B) Animals from A) were sacrificed at the peak of the disease and spinal cord sections were stained with LFB, and antiMAC3 and anti-CD3 antibodies. Quantification of spinal cord demyelination percentage at the different spinal cord levels (upper). Quantification of area occupied by the MAC-3 staining (middle). Quantification of CD3 positive infiltrates at the spinal cord (lower). C) Representative LFB-stained spinal cord sections from the different experimental groups at the lumbar region are depicted. Arrows point at an example of demyelinating lesion considered for the analysis of the LFB staining. Scale bar: $200 \mu \mathrm{m}$.

\subsubsection{Enhanced demyelination in FcyRI-IV knock out animals is isotype dependent}

Our observations concerning disease acceleration and demyelination in the FcpRI-IV knock out animals using low doses of $8.18 \mathrm{c} 5 \operatorname{lgG1}$ aroused the hypothesis that antibody isotypes that had been previously demonstrated to exert disease acceleration effects would not be able to perform the same effects in these animals. Furthermore, efficient demyelinating antibodies could also have higher demyelination potential in absence of the Fcy receptors. To asses this point, WT and FcyRI-IV knock out animals were immunized with 50 $\mu \mathrm{g}$ of $\mathrm{MOG}_{35-55}$ and treated with $50 \mu \mathrm{g}$ of 8.18c5 antibody isotypes shortly before disease onset. Accelerated disease onset upon administration of any antibody isotype was not observed in these animals, supporting the hypothesis that this effect is dependent on the presence of Fcy receptors, and not an antibody isotype-driven process (Figure 25A). In line with the previous results, an enhanced demyelination in FcpRI-IV KO animals treated with the IgG1 isotype control antibody compared to WT animals was observed, supporting the idea that in these animals there is a higher predisposition for demyelination. Additionally, 8.18c5 IgG1 did not induce a significant enhancement of spinal cord demyelination in the WT animals, while it did in the FcyRI-IV KO animals (Figure 25B), also reproducing the previous observations. Administration of 8.18c5 IgE did not enhance demyelination compared to $8.18 \mathrm{c} 5 \operatorname{lgG} 1$, confirming the low demyelination potential of this antibody also in this model. FcyRI-IV KO animals injected with 8.18c5 IgG2b also presented high levels of demyelination, but the degree of demyelination was comparable to the one seen in 8.18c5 IgG1 injected animals. Therefore, the demyelination potential of these two antibody isotypes seems to be comparable at lower doses in this animal model. In addition, quantification of macrophage and T cell infiltration was also not different in this experimental setup (Figure 25A). Taken together, these results indicate that the demyelinating potential of the antibodies can be modified upon ablation of $\mathrm{Fc} \gamma$ receptor expression, but this demyelinating potential is also determined by the isotype of the antibody, similar to the WT situation. 


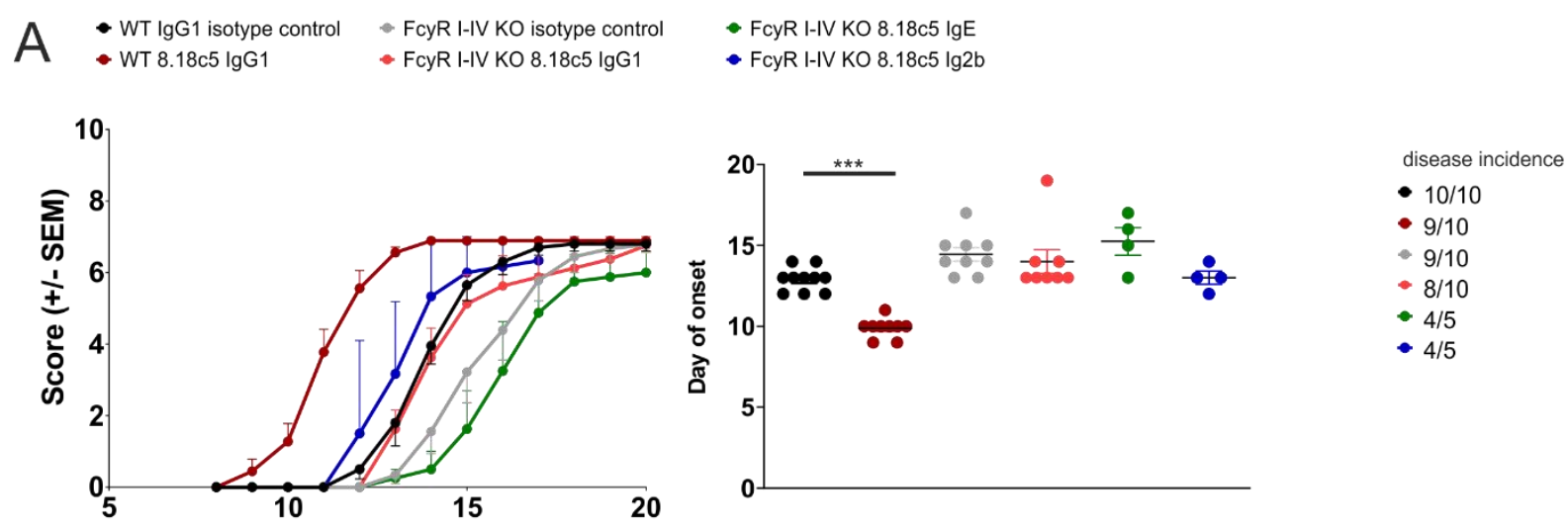

B
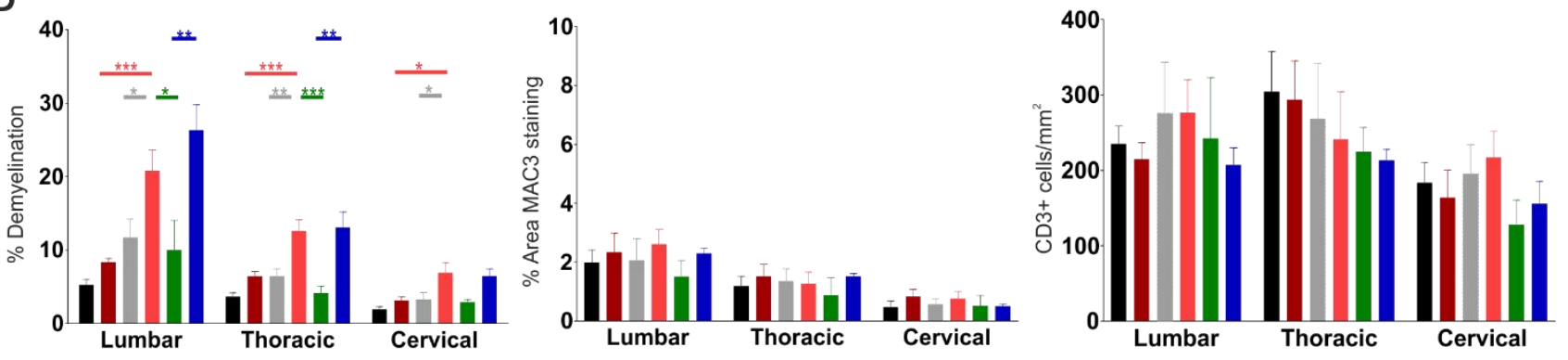

C
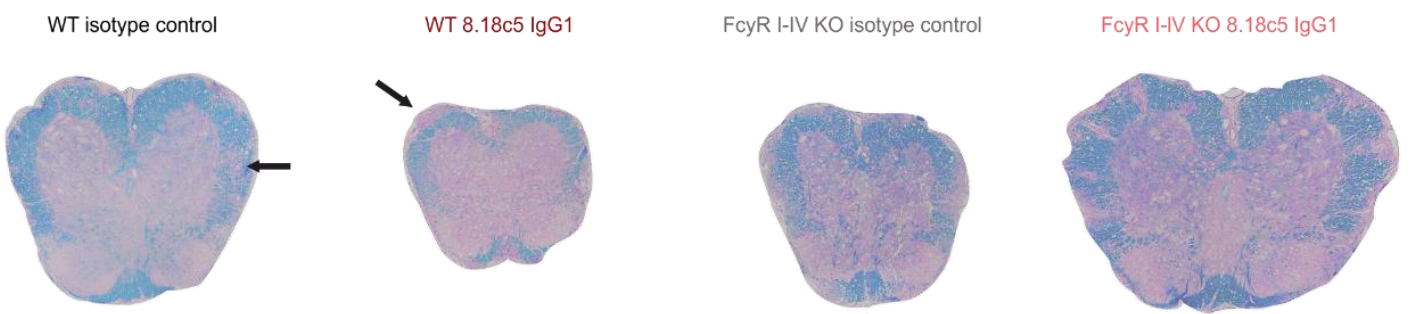

FcyR I-IV KO 8.18c5 IgE

FcyR I-IV KO 8.18c5 IgG2b
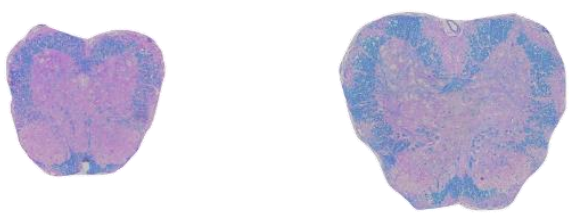

Figure 25. IgGs enhance spinal cord demyelination in Fc receptors alpha chain Knock-out animals more efficiently than other antibody isotypes. C57BI6/J WT or FcyR I-III-IV KO were immunized with $50 \mu \mathrm{g}$ of MOG $35-55$ and intravenously injected with $50 \mu \mathrm{g}$ of 8.18c5 IgG1, IgE, IgG2b or IgG1 isotype control. A) The clinical course of the disease (left), the day of onset (middle) and the disease incidence (right) are depicted. B) Animals were sacrificed at the peak of the disease and spinal cord sections were stained with LFB, and anti-MAC3 and anti-CD3 antibodies. Quantification of spinal cord demyelination percentage at the different spinal cord levels (left). Quantification of area occupied by the MAC-3 staining (middle). Quantification of CD3 positive infiltrates at the spinal cord (right). C) Representative LFB-stained spinal cord sections from the different experimental groups at the lumbar region are depicted. Arrows point at an example of demyelinating lesion considered for the analysis of the LFB staining. Scale bar: $200 \mu \mathrm{m}$. 


\subsection{Enzymatic cleavage of the antibody glycosylation pattern modifies the clinical outcome of EAE}

Further molecular properties of the antibodies have been shown to have crucial roles for their effector function, such as the antibody glycosylation pattern. In particular, glycosylation motifs located at the Asn 297 have been shown to exert crucial effects in the recognition of the Fc fragment by Fc receptors, and for C1q complement subunit binding to the antibodies, consequently triggering the activation of the classical complement pathway during the immune response ${ }^{103-114}$. We investigated whether enzymatic digestion of the glycosylation pattern of the 8.18c5 IgG1 antibody could influence the disease progression of EAE animals. To this end, $100 \mu \mathrm{g}$ of 8.18c5 IgG1 and IgG1 isotype control were processed for glycosylation removal under nondenaturing conditions (Figure 26A). Successful glycosylation removal was confirmed by gel electrophoresis. Enzymatic glycosylation removal did not impair the acceleration effect observed upon administration of 8.18c5 IgG1 compared to isotype control (Figure 26B). However, a slight non-significant decrease in disease severity was observed. These results indicate that modification of other molecular properties of antibodies such as glycosylation pattern can have an effect in our EAE model, but not sufficient to abrogate the observed disease acceleration effects.

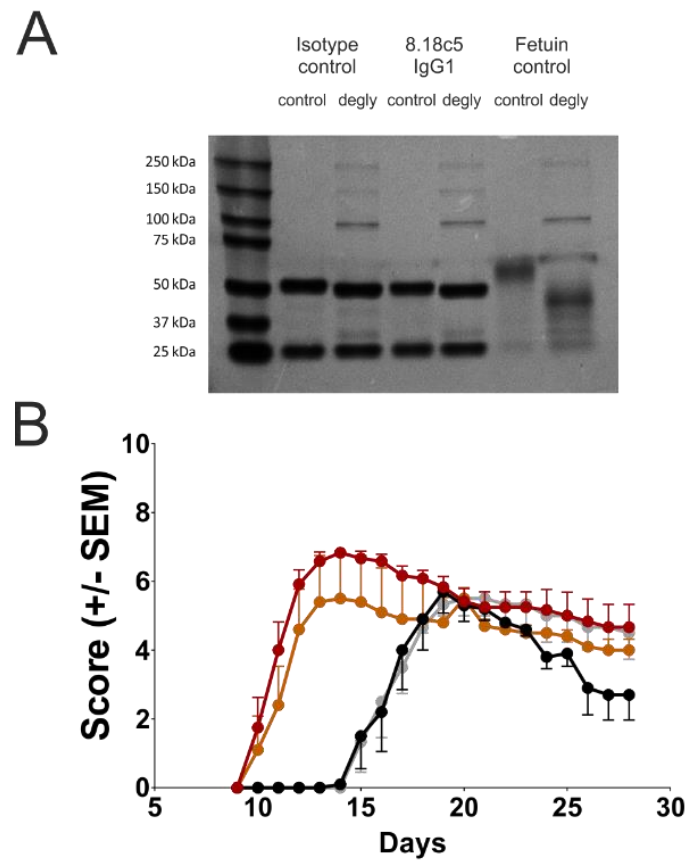

- IgG1 isotype control

- $8.18 \mathrm{c} 5 \lg \mathrm{lg} 1$

- IgG1 isotype control Deglycosilated

- 8.18c5 IgG1 Deglycosilated

disease incidence

- $5 / 5$

- $6 / 6$

- $3 / 4$

- $4 / 6$

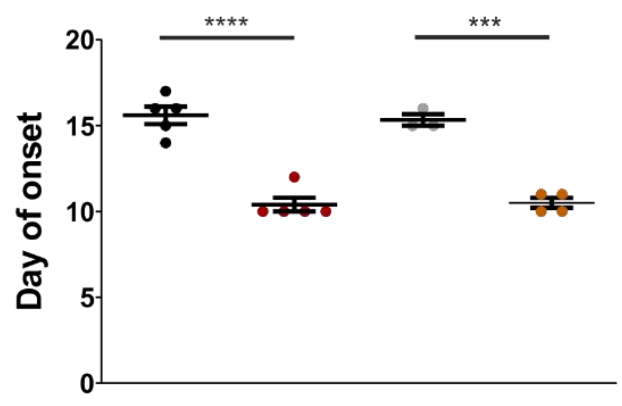

Figure 26. Removal of 8.18c5 IgG1 glycosylation motifs induce a slight decrease of EAE disease severity. A) $100 \mu \mathrm{g}$ of 8.18c5 IgG1 or IgG1 isotype control were deglycosylated under non-denaturing conditions with Protein Deglycosylation Mix II (New England Biolabs). Glycosylation removal of $8.18 \mathrm{c} 5$ IgG1 or IgG1 isotype control was confirmed by gel electrophoresis. B) C57BI6/J animals were intravenously injected with 8.18c5 IgG1 (red), deglycosylated 8.18c5 IgG1 (orange), IgG1 isotype control (black) or deglycosylated IgG1 isotype control (grey) on d8 after immunization with MOG $_{35}$ 55. The clinical course of the disease (left), disease incidence (right upper) and day of onset (right lower) is depicted. 


\section{Discussion}

Although autoreactive CNS-specific effector T cells are thought to be the main cell type responsible for the initiation of MS, increasing evidence suggests that B cells also play an essential role in the disease's pathogenesis, as well as in its animal model $E A E^{115,116}$. The aim of this project was to elucidate the role of the antibody isotype in antibody-mediated effector mechanisms within the CNS. Since antibodies of different isotypes are known to exert different effector functions due to their structure and molecular properties ${ }^{108,103}$, we sought to produce different antibody isotypes through genetic engineering of hybridoma cell lines, ensuring the maintenance of important post-translational modifications for antibody effector function such as the glycosylation pattern. A CRISPR-Cas9 system was designed in order to switch the expressed isotype in the $8.18 \mathrm{c} 5$ and $Z 2$ hybridoma cell lines, which led to the production of different antibody isotypes while preserving their specificity. Using these isotypes, we could show that both antibody-mediated acceleration of EAE onset and CNS demyelination were isotype dependent. Furthermore, we addressed the role of the Fcy receptors mediating antibody effector functions, and we could show that the genetic removal of all FcyRs abrogated the antibody-dependent disease acceleration effect, but potentiated CNS demyelination.

The CRISPR-Cas9 technology has been widely used in recent years in order to modify the genetic architecture of different target organisms and cell lines. Previous reports indicated that Cas 9 upstream targeting of the heavy chain genes codifying for different antibody isotypes lead to class switch in hybridoma cell lines ${ }^{45}$. Following a similar strategy, we were able to switch the isotype expressed by the 8.18c5 and Z2 hybridoma cell lines, which are known to produce demyelinating antibodies against MOG of the IgG1 and IgG2a isotype, respectively ${ }^{84}$. The purified antibodies from switched $8.18 \mathrm{c} 5$ and $Z 2$ cell lines were positive for the desired isotype and additionally they preserved the specificity for MOG, as we confirmed by WB, ELISA and by FACS analysis using T-mMOG cells (Figure 11, 12 and 14). We were able to generate IgE and IgG2b from the 8.18c5 hybridoma, and additionally this system allowed us to produce IgE and IgA in the Z2 hybridoma. Our experiments confirmed the results from previous studies reporting the possibility of driving isotype switch in cell lines while preserving the original antigen specificity.

CSR recombination implies the excision of gDNA regions contained between the $\mathrm{C} \mu$ and the expressed isotype (Figure 2) ${ }^{43}$. Considering the gDNA organization at the heavy chain locus of $8.18 \mathrm{c} 5$ and $\mathrm{Z2}$ hybridoma cell lines, it is not possible to produce the IgM and IgG3 isotypes in the $8.18 \mathrm{c} 5$ hybridoma using our CRISPR-Cas9 system, as well as the $\operatorname{lgM}$, $\operatorname{lgG} 3$, $\lg G 1$ and $\lg G 2 \mathrm{~b}$ antibody isotypes in the $Z 2$ hybridoma by this approach. Therefore, to produce those isotypes, the introduction of gDNA sequences codifying for the heavy chains of these isotypes by gene knock in is required while mutating the first constant exon domain of the next isotype at the same time. However, knock in approaches can induce DNA damage and cell death in a later stage, since long 
homologous regions are necessary for recombination into the target gDNA sequence ${ }^{117,118}$. A CRISPR-Cas9 targeting approach for gene knock in based on 20 nucleotides has been recently published, namely the CRISPRCas9 mediated homology-independent targeted integration (HITI) ${ }^{119}$. The HITI approach ensures the introduction of the desired DNA construct in the forward orientation, enabling the introduction of big DNA fragments without the previous requirement of long recombination regions, resulting in reduced cellular stress that might lead to cell death. Consequently, the HITI approach seems to be a suitable method for the generation of the remaining isotypes that need to be introduced into our hybridoma cell lines.

Within the CNS, antibodies can exert several effector mechanisms leading to CNS inflammation and nervous tissue destruction. These mechanisms include enhanced phagocytosis of immune complexes by meningeal cells, resulting in an increased T cell activation and disease acceleration, and direct binding to myelin, thereby enhancing CNS demyelination ${ }^{85}$. Additionally, antibodies might activate astrocytes and microglia, triggering their release of pro-inflammatory factors, which would induce the recruitment of more immune cells from the blood stream ${ }^{87}$. Taking these antibody effector mechanisms within the CNS into consideration, this study assessed the role of the antibody isotype in disease aggravation and T cell activation, as well as in CNS demyelination.

Linington and collaborators described the so-called "two hit model" for CNS autoimmunity"92,120, where encephalitogenic T cells initiate the inflammation and promote the disturbance of the blood-brain barrier and, as a consequence, anti-MOG autoantibodies would be able to access the CNS and exert damage to the myelin sheaths, aggravating the disease. The increased disease severity has been long considered to be due to enhanced demyelination and CNS tissue destruction, since autoantibodies can damage myelin structures through ADCC or activation of the complement cascade ${ }^{92}$. Although previous studies attributed special emphasis to the demyelinating potential of MOG autoantibodies, recent results from our laboratory showed that the role of demyelination by MOG-specific antibodies was not crucial when applied at low doses. Rather, the presence of MOG-specific autoantibodies accelerated the entry of autoreactive effector T cells into the CNS, leading to an earlier disease onset and disease aggravation ${ }^{87}$. These discrepancies could be explained by the fact that, in comparison to other studies where the administered doses of MOG-antibodies were relatively high (30 $\mu \mathrm{g} / \mathrm{g}$ i.v. and $200 \mu \mathrm{g} / \mathrm{g}$ i.p.), our system is characterized by the administration of relatively low antibody doses (50 $\mu \mathrm{g} / \mathrm{mouse}$ i.v. and $10 \mu \mathrm{g} /$ mouse i.t.). Therefore, our data indicate that, in addition to the role of demyelination in disease exacerbation, further mechanisms through which autoantibodies promote EAE development should be considered. For that reason, we sought to address the role of antibodies in both the disease acceleration effect and in CNS demyelination, taking the autoantibody isotype additionally into account. 
Our experiments in the active and transfer EAE models show that the disease acceleration effect was inducible upon administration of MOG-specific IgGs of different subclasses (IgG1, IgG2a and IgG2b), but not by IgE and IgA (Figure 15, 16, 18 and 21). Furthermore, animals injected with IgGs showed an earlier disease onset both when MOG-specific IgGs were administered i.v. and i.t., independently of the IgG subclass. These results indicate that, on the one hand, autoantibody-driven disease acceleration seems to be isotype dependent. On the other hand, these experiments confirm that the disease accelerating effect of MOG antibodies is a phenomena occurring within the CNS rather than in the periphery ${ }^{87}$, in contrast to other studies which highlight the importance of MOG-specific antibodies for T cell priming at the peripheral and CNS draining lymph nodes ${ }^{86}$. These studies reported disease-accelerating effects of MOG autoantibodies, where 2D2 and WT recipients were injected with serum from animals whose BCR is restricted to MOG (Th mice) $)^{86}$. The serum of these animals contains antibodies against MOG of different isotypes, being IgG2a the most frequent antibodies found in these samples ${ }^{32,87}$, and $58 \%$ of double transgenic animals harbouring MOG-specific $\mathrm{T}$ and B cells develop spontaneous disease ${ }^{121,18}$. The crucial role of MOG-specific antibodies regarding disease acceleration and spontaneous disease development has been previously described using animals harbouring mainly antibodies of the IgG isotype, providing further support to our observations. To our knowledge, in the current study it is the first time that the role of specific and well-defined isotypes in triggering diseasemodifying effects has been investigated.

We reasoned that different clinical outcomes upon administration of different isotypes could be due to differences in autoreactive T cell re-activation within the CNS. Binding of autoantibodies to their target antigen, and subsequent internalization of these immune complexes by APCs in a Fc-receptor dependent-manner, could have a more efficient reactivation of autoreactive effector T cells within the CNS as a consequence, leading to an earlier disease onset. In order to investigate this hypothesis, the T-cell activation in WT animals transferred with T cells from Nur77-GFP animals was measured by flow cytometry. In this set up, activated T cells are fluorescently detectable by FACs upon TCR stimulation, but not other stimuli such as cytokine or chemokine signalling ${ }^{101}$. Increased percentages of activated $\mathrm{T}$ cells in the spinal cord parenchyma and meninges were found upon administration of MOG-specific IgG1 at disease onset (Figure 19). Furthermore, administration of IgG2b led to a non-significant tendency towards an enhancement of the percentage of CD4+ GFP T cells. Nevertheless, since IgG2b induced disease acceleration in the EAE experiments, further experiments are required to clarify the role of $\operatorname{lgG} 2 \mathrm{~b}$ in $\mathrm{T}$ cell activation within the CNS. In contrast, administration of MOG-specific IgE did not lead to higher percentages of activated T cells within the meninges and spinal cord compared to its respective isotype control. Of note, antibody isotypes have different affinity for the Fc receptors, which might help to explain differences regarding T cell reactivation. For example, while IgG1 antibodies are reported to efficiently bind FcyRs but not to efficiently fix complement, IgG2b antibodies are known to efficiently perform in both processes ${ }^{122}$. This opens the possibility that IgG1-myelin immune 
complexes are mainly captured by APCs which would present antigen more efficiently to autoreactive T cells, being IgG1 only minimally involved in complement-related mechanisms ${ }^{123}$. In contrast, IgG2b antibodies would be able to support both mechanisms, being not as efficient as IgG1 in T cell reactivation, but maybe having a more important role in complement-dependent CNS demyelination.

Effector T cells produce cytokines upon activation that trigger the recruitment of other immune cells, promoting disease development ${ }^{87}$. Previously, it was reported that the disease acceleration effect was accompanied by an enhanced cytokine production in the CNS of animals administered with IgG1 autoantibodies ${ }^{87}$. This raised the question whether the enhanced cytokine production was a consequence of higher numbers of activated T cells infiltrating the CNS, or rather due to higher cytokine expression levels of individual activated T cells upon administration of MOG isotypes. Our results showed that, although sorted GFP positive T cells produced higher amounts of pro-inflammatory cytokines compared to non-activated sorted GFP negative T cells, similar amounts of cytokine transcripts were found in GFP+ activated T cells among all the different antibody isotypes and their respective isotype controls (Figure 19). These results indicate that enhanced cytokine production of individual cells upon administration of different antibody isotypes is not crucial for disease acceleration, but rather the enhanced numbers of CD4+ T cells reactivated within the CNS. However, the Nur77 GFP reporter might not be a suitable system with which evaluate the peak of cytokine production, since this has been reported to be measurable 30 min after stimulation of human CD4+ and CD8+ T cells, peaking at 3-4 hours post stimulation ${ }^{124,125,126}$. In contrast, detection of GFP in activated Nur77 GFP T cells is possible after two hours and has its maximum emission 12 to 24 hours after antigen stimulation in vitro ${ }^{101}$. Therefore, it is possible that the peak of GFP expression and cytokine production are not synchronised, preventing us from detecting differences upon administration of different antibody isotypes.

8.18c5 IgE, and $Z 2 \operatorname{lgE}$ and $\lg A$ did not have an effect in disease acceleration (Figure 16, 17 and 21). Furthermore, increased numbers of activated T cells were not observed when 8.18c5 IgE was administered (Figure 19). Although further experiments are required in order to confirm these results, it seems that IgE and IgA isotypes cannot accelerate disease onset and aggravate EAE. The absence of the aforementioned effects upon administration of these antibody isotypes could be due to their particular structural and functional properties.

Regarding IgE, murine half-life of IgE is short in comparison to other antibody isotypes ${ }^{62}$, which may explain the absence of pathogenic effects. Several mechanisms have been proposed to explain the short half-life of IgE, such as a higher sensitivity of IgE for proteases or competition with IgGs for binding available FcRn molecules at the endosomal compartments ${ }^{127}$. In order to substantiate that the absence of disease acceleration upon administration of IgE was related to the isotype and not to its short half-life, we administered IgE on three consecutive days. However, triple administration of IgE was not sufficient to trigger 
disease acceleration, nor was administration of higher doses of IgE, independently of the administration route (Figure 17, 21 and 22). Furthermore, while fluorescently labelled 8.18c5 IgG1 has been shown to be present within the CNS 4-5 days after administration ${ }^{87}$, there is no available data regarding the arrival of MOG-specific IgE to the CNS, and therefore 2-photon experiments using labelled IgE antibodies are necessary to investigate the role of this isotype in EAE.

IgE triggers its effector mechanisms through binding to the $\mathrm{F} c \varepsilon R I$, which is expressed by basophils and mast cells, and FceRII, which is expressed by $T$ and B cells as well as different myeloid cell populations ${ }^{128,129}$. Crosslinking of the FceRI lead to degranulation of basophils and mast cells, and activation of FceRII leads to suppression of IgE production by B cells, but it also may be involved in antigen presentation to allergen specific $\mathrm{T}_{\text {cells }}{ }^{130}$. Since we did not observe disease acceleration upon administration of IgE, the potential role of FcERII in our disease model might be not crucial for disease exacerbation. However, IgE might exert diseasemodifying effects through mast cell and basophils degranulation in EAE. Mast cells are known to regulate vascular permeability, and they are found in close relationship with BBB vasculature and with nerves ${ }^{131}$. Mast cells can not only be found in the non-inflammed CNS tissue, such as in the thalamus and hippocampus, but they have also been found in demyelinating lesions of MS patients, and also as transcripts of tryptase, which can promote demyelination ${ }^{131,132}$. Some authors have claimed a pathogenic role for mast cells using mast celldeficient mice (c-kit KO), since these animals had an ameliorated disease progression ${ }^{133,134}$. In these studies, bone marrow mononuclear cell transplantation reconstituted mast cell populations and rescued EAE severity. However, c-kit deficiency affects mast cells but also induce several defects including lymphocyte development, indicating that this model might not be a suitable one for studying mast cell biology. Other authors recently tried to address the role of mast cells in EAE in a more direct way using KO animals for carboxypeptidase $A$ and for c-kit. Their results showed that both KO models are equally susceptible to MOG-induced EAE, presenting similar clinical outcome and histpathological profiles and, consequently, suggesting a minor role for mast cells in the EAE model ${ }^{135}$. However, recent publications indicated a protective role of mast cells and basophils in $E A E$, since treatment with anti-FceRI antibodies enhanced EAE severity through a polarization of the immune response towards a Th1 and Th17 phenotype ${ }^{136}$. Future experiments in our model using the knock out mice for the Fc common- $\gamma$ chain, which is also part of the $\mathrm{Fc}_{\mathrm{CR}}{ }^{96}$, are required to further study the role of IgE in autoimmunity.

In contrast to humans, IgA is together with IgE the least abundant antibody isotype found in young and adult mice $^{50}$, and it also has a short half-life ${ }^{62}$. Since we did not observe disease-modifying effects upon administration of IgA (Figure 21), it is possible that this antibody isotype does not reach the CNS in enough quantities in order to exert these effects. Therefore, 2-photon in vivo studies using labelled IgA are required 
to test whether this isotype is able to reach the CNS, as well as administration of this isotype on consecutive time points.

IgA is known to play an important role in gut homeostasis through its binding to the plgR and the Fc $\alpha / \mu R$. While plgR mediate the transport across the epithelium of slgA in the gut, $F c \alpha / \mu R$ is internalized upon crosslinking with $\operatorname{IgM}$ and $\operatorname{IgA}$ on B cells and macrophages, and it presumably participates in antigen presentation ${ }^{137}$. Intestinal IgA-producing plasma cells have been recently reported to dampen EAE neuroinflammation through the production of IL-10, having therefore an anti-inflammatory effect in the CNS. However, these studies did not find significant evidence of IgA antibody-driven disease modulatory effects since irradiated $\mathrm{B}$ cell deficient animals $\left(\mathrm{Jht}^{-/}\right)$reconstituted either with IgA-deficient BM or WT BM developed a similar EAE severity ${ }^{138}$. The presence of IgA autoantibodies against native myelin basic protein in the CNS has been recently reported in MS patients ${ }^{139}$, and the role of the gut microbiome within MS pathology is progressively gaining attention ${ }^{140}$. Alterations in the gut microbiome lead to different outcomes in the EAE model. For example, mice kept in germ-free conditions present a decreased EAE incidence, fewer Peyer's patches and reduced production of $\lg \mathrm{A}^{140,141}$. In this line, antibiotic treatment with kanamycin, colistin and vancomycin one week before immunization reduced EAE severity, together with a decrease of IFN $\gamma$, TNF $\alpha$, IL6 and IL-17 production ${ }^{140}$. Additionally, B-cell development, activation and differentiation have been shown to be influenced by microbial products and gut microbiota, since stimulation of Toll-like receptors in the intestinal lumen trigger the release of BAFF and APRIL, leading to B cell activation and promoting CSR towards IgAproducing plasma cells ${ }^{142,143}$. Finally, IgA deficiency is the most common immunoglobulin deficiency in humans, which shows variances between different geographical regions, but it is also associated with autoimmunity ${ }^{144}$. Taking all these factors into consideration, further experiments are required to understand the role of IgA in our model.

The initial reports that pointed towards the role of $B$ cells in EAE made also reference to the participation of IgM antibodies in this model. Rats administered with anti-lgM antibodies failed to develop EAE when they were administered with guinea pig spinal cord homogenate or purified MBP ${ }^{145}$. This EAE resistance was counteracted upon administration of serum containing anti-MBP into anti-IgM treated animals, indicating that although IgM is not required in order to generate autoreactive effector T cells, this isotype facilitates the development of the disease ${ }^{146}$. On the other hand, IgM-dependent protection against autoimmunity has been also suggested, since patients with selective IgM deficiency are more prone to develop other autoimmune diseases ${ }^{147,148}$. Furthermore, Fc $\mu \mathrm{R}$ KO animals produce less antibodies in primary and memory immune responses and, as they become older, they produce more autoantibodies than control animals ${ }^{149}$. However, in our experimental setup, antibodies are administered briefly before disease onset, and therefore it is not likely that the reported tolerance induction mechanisms of IgM could take place. Although IgM present a half- 
life of two days in adult mice ${ }^{62}$, 2-Photon in vivo experiments using labelled IgM would also be required in order to test whether this isotype can reach the CNS, since given its high molecular weight, it is possible that IgM cannot cross the blood-brain barrier even when disrupted.

In this study, we additionally sought to address the question of whether Fcy receptors play a role in autoantibody-dependent disease acceleration in EAE. Our experiments revealed that elimination of the FcRn, Fcy type I activating receptors I and III, and the common- $\gamma$ chain plus Fc receptor IIB, did not modify the performance of $8.18 \mathrm{c} 5 \lg \mathrm{g} 1$ regarding disease acceleration. However, this effect was abrogated in FcYRI-IV KO animals in an isotype-independent manner (Figure 24 and 25), suggesting that the expression of FcyRs is crucial for an earlier disease manifestation.

FcRn plays important roles extending the half-life of immunoglobulins, specifically that of IgGs, since they are the most common antibodies found in serum and presumably they occupy the majority of FcRn units available ${ }^{113,127}$. Furthermore, the FcRn has been implicated in antigen processing by dendritic cells when upon uptake of immune complexes, avoiding their degradation in the lysosome compartment ${ }^{63}$. This is probably not the case in our experimental setup, since elimination of the FcRn was not sufficient to abrogate IgG1-driven disease acceleration effects (Figure 24). These results indicate that the effector mechanisms of IgG1 are triggered in a relatively short timeframe, which does not necessarily require the interaction with FcRn. An interesting follow-up experiment is the evaluation of $\mathrm{T}$ cell activation in FcRn KO mice upon adoptive transfer of cells from Nur77-GFP animals, since it is possible that upon administration of IgG1 antibodies similar amounts of activated T cells could be found in the CNS of these animals, even when the half-life of this immunoglobulin is compromised.

Regarding the $\mathrm{KO}$ animals for the FcyRI and III, other studies found EAE disease amelioration upon knock out of FcyRIIII50, and no effects compared to WT upon ablation of $\mathrm{Fc}^{15 R I^{151}}{ }^{15}$. In line with these latter studies, we did not find an abrogation of EAE disease acceleration (Figure 24). Based on recent results from our department, it seems that compensatory mechanisms concerning the expression among the different FcyRs could explain the continuation of the antibody-driven disease acceleration effect ${ }^{109}$.

The common- $\gamma$ chain FcyR KO model has been the most frequently used FcR KO model in the previous years, although conflictive results concerning the role of activating FcyRs in EAE susceptibility have been published using this model ${ }^{88,152}$. In these animals, expression of the activating FcyRs alpha chains is compromised but not completely abrogated ${ }^{96}$, which could be sufficient to mediate internalization and processing of immune complexes, leading to disease acceleration in our model. In fact, our experiments using common- $\gamma$ chain Fc $\gamma R$ KO animals and carrying additionally a KO for the FcyR IIB showed that knock out of these elements is not 
sufficient to abrogate the acceleration effect (Figure 24). Therefore, we could support other results which also found disease acceleration effects upon administration of $8.18 \mathrm{c} 5$ in this $\mathrm{KO}$ mode ${ }^{96}$.

In summary, our results indicate that the disease acceleration effect in the EAE model is isotype dependent, where IgGs play a crucial role, and it requires necessarily the expression of FcyRs in order to be triggered.

Regarding the other antibody-effector mechanism tested in this study, namely CNS demyelination, autoantibodies can target a cytotoxic attack to myelin structures through ADCC or activation of the complement cascade, leading to enhanced demyelination and tissue destruction ${ }^{38,84}$. Previous results from our department showed that animals administered with low doses of $8.18 \mathrm{c} 5 \operatorname{lgG} 1$ did not show enhanced demyelination briefly after disease onset, although the disease acceleration effect was still present ${ }^{87}$. However, several studies reported a high demyelinating potential of MOG-specific autoantibodies ${ }^{38,153}$, as well as upon administration of $150 \mu \mathrm{l}$ of serum from Th mice at later disease stages in 2D2 animals ${ }^{86}$. In comparison to these studies, our model is characterized by the administration of low quantities of autoantibodies. Therefore, we sought to address whether CNS demyelination could be affected by increasing doses of autoantibodies at the peak of the disease, as well as the role of different antibody isotypes targeting MOG regarding CNS demyelination.

We could show that low doses of 8.18c5 IgG1 did not enhanced demyelination, but in line with previous studies, higher doses of this antibody isotype led to enhanced demyelination of the spinal cord (Figure 20, 22 and Table 5). Irrespectively of the dose applied, all animals treated with 8.18c5 IgG1 presented an accelerated disease onset, suggesting that demyelination and disease acceleration are two independent mechanisms of autoantibodies. Furthermore, antibody-dependent demyelination seems not only to be modulated by the concentration of the antibody, but also by the isotype (Figure 20, 22 and Table 5). In this regard, CNS damage was more pronounced upon administration of $8.18 c 5 \operatorname{lgG} 2 \mathrm{~b}$ and $Z 2 \operatorname{lgG} 2 \mathrm{a}$, while $8.18 \mathrm{c} 5 \operatorname{lgE}, \mathrm{Z2} \operatorname{lgE}$, and Z2 IgA did not have an effect on spinal cord demyelination. Intrathecal administration of different antibody isotypes showed similar results (Figure 22), but only in case the antibodies were administered at early time points rather than at the peak of the disease (Figure 23). Taken together, these data indicate that IgGs are the only antibody isotypes with the capacity to trigger an enhancement of CNS demyelination but with differential outcome between the IgG subclasses.

It seems that the threshold required for IgG1 in order to exert disease acceleration and CNS demyelination is different, being more efficient in accelerating EAE onset than triggering demyelination and CNS damage through $A D C C$ and CDC. This could be a consequence of IgG1 molecular properties, since IgG1 antibodies are more efficient in binding to FcyRs than in complement fixation compared to other antibody isotypes ${ }^{122,64}$. Supporting this idea, previous studies suggested that the demyelinating potential of MOG autoantibodies is 
based on their ability to fix complement ${ }^{153}$, being $\mathrm{Z2}$ IgG2a antibodies more efficient triggering SC demyelination than $8.18 \mathrm{c} 5 \operatorname{lgG} 1$. In line with this, it is known that complement fixation is isotype-dependent, given that $\lg G 2 a$ and $\lg$ G2b are more efficient in this process than $\lg G 1$ antibodies ${ }^{64}$. Therefore, complement fixation seems to be a good candidate to investigate the exact mechanism of isotype-dependent demyelination in our model.

Increasing doses of IgE and IgA did not lead to enhanced demyelination, as well as direct administration of 8.18c5 IgE into the CNS (Figure 20, 22 and Table 5). Since CDCC and CDC are most likely driving CNS demyelination in our mode $\left.\right|^{84}$, it is not surprising that we did not observed IgE-mediated demyelinating effects, as this antibody is reported not to be able to fix complement ${ }^{154}$. However, infiltrating cells expressing nonoccupied FcERI molecules in their surface could recognize immune complexes containing myelin debris and IgE, and release pro-inflammatory cytokines and proteases which can exert further damage within the CNS tissue through $A D C C^{155,156}$. This seems not to be the case, since FcERIs non-occupied by IgE have a half-life of 24 hours in vitro, therefore the binding of IgE to this receptor is necessary in order to avoid the recycling of the receptor 68,157 . In vivo 2-Photon studies and FACs analyses tracking labelled IgE are required to substantiate our evidence of a lack of effect on demyelination of this antibody isotype seen so far in our model. On the other hand, IgA does not trigger the activation of the classical complement pathway, but it is known to activate the alternative complement pathway and the mannose lectin complement pathway ${ }^{73-74}$, which also have been reported to play a role in the EAE model ${ }^{158}$. Given the short half-life of $\operatorname{IgA}$, it is possible that larger amounts are required to trigger its demyelinating potential. Furthermore, in this study we did not test the effect of direct administration of IgA into the CNS, which could lead to enhanced demyelination by CDCC or CDC.

Taking the potential role of complement in our model into consideration, the generation of MOG-specific IgM and IgG3 in the 8.18c5 and Z2 hybridoma cell lines would be particularly interesting. These two antibody isotypes possess a particular immunostimulatory capacity including a positive feedback loop for antibody responses, which seems to be complement-dependent ${ }^{123,159}$. In this line, both antibodies are known to efficiently bind to complement ${ }^{123,160,161}$, and IgG3 can oligomerize recruiting more easily C1q subunits ${ }^{162}$. Consequently, in our model these antibody isotypes could significantly boost spinal cord demyelination through CDCC and CDC, and perhaps by the establishment of a positive feedback loop of antibody production. Concerning the function of IgM and IgG3 in a different context, in other EAE models using rhesus, marmoset and cynomolgus monkeys, EAE development was correlated with high anti-rhMOG IgM responses, indicating that the IgM-driven humoral response is essential in these models for disease induction. Finally, in MS patients it is described that intrathecal IgM production correlates with a severe disease course, leading to the appearance of more spinal cord lesions ${ }^{163}$. 
Variations in CNS demyelination was not accompanied by further infiltrating T cells (Figure 20), but upon higher doses of IgG2b and IgG2a, increased numbers of cells positive for MAC3 were present, indicating that these antibody isotypes can induce the recruitment of macrophages to the CNS, or activate microglial cells more efficiently. Characterization by qRT-PCR of cytokines such as TNF $\alpha$, IL-1 $\beta$, IL-6, and macrophagerecruiting chemokines such as CXCR5 and CCR5, in total CNS tissue, as well as per cell analyses of infiltrating macrophages and microglia cells, could help us to understand the role of IgG2b and IgG2a in macrophage recruitment. Finally, since histological approaches are conditioned by the analysed sections, which do not necessarily always reflect the infiltration status of the tissue, evaluation of macrophage infiltration should be further characterized by FACs staining of $C D 11 b+C D 45+$ populations to be able to get a better overview of the infiltration of myeloid cells.

The role of Fcy receptors in autoantibody-dependent demyelination was also addressed in this study. Our experiments revealed that elimination of the FcRn, Fc $\gamma$ type I activating receptors I and III, and the common- $\gamma$ chain plus Fcy receptor IIB, did not modify the performance of 8.18c5 IgG1 in SC demyelination (Figure 24). However, FcYRI-IV KO animals showed enhanced demyelination compared to WT animals when administered with isotype control, as well as enhanced demyelination upon administration of $50 \mu \mathrm{g}$ of $8.18 \mathrm{c} 5$ compared to FcyRI-IV treated with isotype control (Figure 24). Enhancement of demyelination in FcyRI-IV KO animals was also isotype dependent, since both IgG1 and IgG2b exerted higher demyelination than IgE (Figure 25), recreating a similar situation to the one observed in WT animals. Interestingly, in this setup both $50 \mu \mathrm{g}$ of $\lg \mathrm{g} 1$ and IgG2b exerted comparable CNS demyelination, suggesting that a maximum demyelination plateau can be reached in our model with no further possible exacerbation. Whether other antibody isotypes perform differently in FcyRs I-IV KO animals needs to be addressed using further antibody isotypes derived from the $8.18 c 5$ and $Z 2$ hybridoma cell lines.

Our data suggest that antibody-dependent disease acceleration and demyelination seem to be independent mechanisms. However, our experiments using the FcyRI-IV KO model suggest that these two mechanisms are closely interrelated. The abrogation of the disease acceleration effect and the same time the enhanced demyelination observed in FcYRI-IV KO animals could indicate that, since antibodies cannot bind to these receptors anymore, the uptake of immune complexes by APCs would be limited. Thereby, a more efficient reactivation of T cells would no longer be possible, leading to an abrogation of the disease accelerating effect. However, these antibodies would now preferentially bind to myelin and therefore could promote CNS demyelination through the only available system, the complement cascade, which has been extensively implicated in myelin destruction in the EAE model ${ }^{164}$. This could also imply that the threshold required for a particular isotype to fix complement would be more easily reached, which is supported by the enhanced demyelination found in FcyRI-IV KO animals treated with isotype control. Our immunization protocol using 
$M \mathrm{MG}_{35-55}$ peptide results in the production of very low endogenous MOG-autoantibody titers ${ }^{85}$. Consequently, in case that the FcyRs are not present, the threshold for antibodies to fix complement would be lowered and a small titer of antibodies upon immunization would be sufficient to promote CNS demyelination through CDCC or CDC. Murine IgG1 represents a good example for this interpretation, since it is known that this antibody isotype is more efficient binding to its FcyRs than fixing complement ${ }^{89,123,165}$. However, low amounts of this antibody isotype enhanced dramatically CNS demyelination when FcyRs expression was abrogated, indicating that the threshold for this isotype to trigger myelin damage is lowered in this context.

Furthermore, in the EAE model a crucial role has been shown for complement in autoantibody-mediated demyelination which seems to be independent of activating Fcy-receptors ${ }^{164}$. This study showed that KO animals for the complement subunit C1q, which triggers the activation of the classical complement pathway, did not show enhanced demyelination upon administration of $8.18 \mathrm{c} 5$, while WT animals and FcR common- $\gamma$ chain $\mathrm{KO}$ animals did. In addition, amelioration of other autoimmune diseases has been reported upon specific block of the different complement pathways ${ }^{90}$. Consequently, it is likely that the potential effector mechanisms implicated in CNS demyelination in the EAE model are CDCC and CDC, where the complement cascade would be triggered upon binding of autoantibodies to MOG. In our model, complete inhibition of the complement cascade could abrogate the IgG2b- and IgG2a-driven enhancement of demyelination, while IgGdependent disease acceleration could still be present or slightly ameliorated. Nevertheless, further experiments using $\mathrm{KO}$ animals for the complement subunit $\mathrm{C} 1 \mathrm{q}$, as well as pharmacological approaches using inhibitors for the different complement pathways are required to clarify the importance of the isotype in complement-mediated demyelination.

Further antibody molecular properties such as glycosylation influence antibody effector mechanisms ${ }^{114}$. Although a difference in the clinical outcome of EAE was not observed upon administration of deglycosylated 8.18c5 and isotype control in our experiments (Figure 26), further experiments are necessary to confirm these results. Moreover, since EAE clinics do not necessarily correlate with histopathological marks such as spinal cord demyelination ${ }^{87,3}$, further studies are required to confirm the effect of complete deglycosylation in our EAE model. Additionally, removal of specific residues at the antibody Asn 297 glycosylation site has been reported to modify the outcome of the immune response ${ }^{166,167}$. For example, while sialylation removal promote anti-inflammatory responses, $A D C C$ is boosted by removal of fucose. Therefore, our observations concerning antibody effector mechanisms such as disease acceleration or demyelination could be influenced by alterations in antibody glycosylation patterns.

In a previous report ${ }^{84}$, it was shown that the antibodies derived from the hybridoma cell lines $8.18 \mathrm{c} 5$ and $\mathrm{ZZ}$ exert different effector functions and differ in their demyelinating capacity. On the other hand, these antibodies are of a different isotype, but they also do not exactly bind to the same epitope at the FG loop of 
MOG $^{104,105}$. Therefore, it remained unclear whether the difference in effector function is due to the isotype or to the epitope specificity. With our approach, it is in principle possible to clarify this issue. In this study, the role of MOG-specific IgE antibodies derived from both hybridoma cell lines was addressed. In both cases, antibodies of the IgE isotype were not able to exert disease-modifying effects, either in the active EAE model or in the transfer EAE model. Unfortunately, antibodies of exactly the same isotype with the 8.18c5 and Z2 specificity could not be directly compared, but 8.18c5 IgG2b and Z2 IgG2a showed striking similarities both in respect to disease acceleration and demyelination. It therefore seems that the isotype rather than fine epitope specificity is crucial for the prediction of the effector mechanisms preferentially used by MOG-specific antibodies. Production of the remaining antibody isotypes from both hybridoma cell lines, and further experiments comparing systematically these isotypes, is required in order to finally address this question. 8.18c5 Fab fragments could not exert disease-modifying effects, and failed to accelerate the disease, underscoring that the relevant effector mechanisms are strictly dependent on the Fc portion of the antibodies and that the effects were not due to simple binding of the antibodies to their cognate antigen ${ }^{109}$. In addition, several publications reported previously that the specificity of antibodies can be modified upon $\operatorname{CSR}^{168,169}$. This would provide a scenario were CSR could induce fine modifications at the antigen binding site, making antibodies more efficient recognizing their targets and helping to explain the preference for the production of certain isotypes within concrete immune responses (such as viruses or helmints). This is probably not the case in our model, since our CRISPR-Cas9 based system is most likely not triggering the natural CSR process, but rather inducing a DNA DSB which functional consequence is uniquely a switch of the isotype.

Finally, ADCC and CDC are important FcR-mediated mechanisms in our model ${ }^{170,28}$. Antibodies binding directly to myelin could be recognized by macrophages, neutrophils and other Fc receptor expressing cells ${ }^{66}$, targeting myelin destruction. Generation of antibodies derived from the 8.18c5 and Z2 hybridoma cell lines carrying mutations at the $\mathrm{CH} 2-\mathrm{CH} 3$ region (where the Fc receptor binding site is located) will provide us a useful tool with which to further investigate the triggering of $A D C C$ in our model ${ }^{171,172}$. Regarding CDCC and CDC, mutations at the complement binding site in the $8.18 \mathrm{c} 5$ and $Z 2$ hybridoma cell lines will give us insights into the role of complement in our model ${ }^{89,173}$, since a relationship between complement and demyelinating antibodies concerning demyelination as an effector mechanism has been previously reported ${ }^{84}$. These studies showed that $\mathrm{Z2}$ IgG2a antibodies are more efficiently fixing complement and demyelinating rat CNS tissue than $8.18 \mathrm{c} 5 \lg \mathrm{l} 1$ antibodies, which goes in line with the literature regarding isotype-dependent complement fixation capacity, since $\lg M, \lg G 3, \lg G 2 a$ and $\lg G 2 b$ are known to fix complement more efficiently than $\lg G 1$, $\operatorname{IgA}$ and IgE in mice ${ }^{122,165,162}$. Therefore, production of these additional tools would help us to understand the relationship between FcR-mediated mechanisms and complement-dependent mechanisms in our model. 
In summary, antibodies exert different pathogenic mechanisms depending on their molecular properties in the EAE model, with the isotype being the main variable preferentially investigated is this study. Based on their affinity to FcRs and their ability to fix complement, antibody isotypes exert their effector mechanisms preferentially through one of these two systems, which can be balanced towards one or the other when some of these molecular cues are not available. 


\section{References}

1. Sospedra, M. \& Martin, R. Immunology of Multiple Sclerosis. Semin. Neurol. 36, 115-127 (2016).

2. Stadelmann, C., Wegner, C. \& Brück, W. Inflammation, demyelination, and degeneration - Recent insights from MS pathology. Biochim. Biophys. Acta - Mol. Basis Dis. 1812, 275-282 (2011).

3. Constantinescu, C. S., Farooqi, N., O'Brien, K. \& Gran, B. Experimental autoimmune encephalomyelitis (EAE) as a model for multiple sclerosis (MS). Br. J. Pharmacol. 164, 1079 (2011).

4. Lucchinetti, C. et al. Heterogeneity of multiple sclerosis lesions: implications for the pathogenesis of demyelination. Ann. Neurol. 47, 707-17 (2000).

5. O'Gorman, C., Lucas, R. \& Taylor, B. Environmental risk factors for multiple sclerosis: a review with a focus on molecular mechanisms. Int. J. Mol. Sci. 13, 11718-52 (2012).

6. Munger, K. L. et al. Vitamin D intake and incidence of multiple sclerosis. Neurology 62, 60-5 (2004).

7. Mowry, E. M. et al. Vitamin D status is associated with relapse rate in pediatric-onset MS. Ann. Neurol. 67, NA-NA (2010).

8. Sharif, K., Amital, H. \& Shoenfeld, Y. The role of dietary sodium in autoimmune diseases: The salty truth. Autoimmun. Rev. 17, 1069-1073 (2018).

9. Wingerchuk, D. M. Smoking: effects on multiple sclerosis susceptibility and disease progression. Ther. Adv. Neurol. Disord. 5, 13-22 (2012).

10. International Multiple Sclerosis Genetics Consortium et al. Genetic risk and a primary role for cellmediated immune mechanisms in multiple sclerosis. Nature 476, 214-219 (2011).

11. Wekerle, H. Lessons from multiple sclerosis: models, concepts, observations. Ann. Rheum. Dis. 67, iii56-iii60 (2008).

12. Gold, R., Linington, C. \& Lassmann, H. Understanding pathogenesis and therapy of multiple sclerosis via animal models: 70 years of merits and culprits in experimental autoimmune encephalomyelitis research. Brain 129, 1953-1971 (2006).

13. Burrows, D. J. et al. Animal models of multiple sclerosis: From rodents to zebrafish. Mult. Scler. J. 25, 306-324 (2019).

14. Jäger, A., Dardalhon, V., Sobel, R. A., Bettelli, E. \& Kuchroo, V. K. Th1, Th17, and Th9 Effector Cells Induce Experimental Autoimmune Encephalomyelitis with Different Pathological Phenotypes. J. Immunol. 183, 7169-7177 (2009).

15. Ben-Nun, A., Wekerle, H. \& Cohen, I. R. The rapid isolation of clonable antigen-specific T lymphocyte lines capable of mediating autoimmune encephalomyelitis. Eur. J. Immunol. 11, 195-199 (1981).

16. Pesic, M. et al. 2-photon imaging of phagocyte-mediated T cell activation in the CNS. J. Clin. Invest. 123, 1192-1201 (2013).

17. Lodygin, D. et al. A combination of fluorescent NFAT and H2B sensors uncovers dynamics of T cell activation in real time during CNS autoimmunity. Nat. Med. 19, 784-790 (2013).

18. Krishnamoorthy, G., Lassmann, H., Wekerle, H. \& Holz, A. Spontaneous opticospinal encephalomyelitis in a double-transgenic mouse model of autoimmune T cell/B cell cooperation. $J$. Clin. Invest. 116, 2385-2392 (2006).

19. Whitham, R. H. et al. Lymphocytes from SJL/J mice immunized with spinal cord respond selectively to a peptide of proteolipid protein and transfer relapsing demyelinating experimental autoimmune encephalomyelitis. J. Immunol. 146, 101-7 (1991).

20. McRae, B. L. et al. Induction of active and adoptive relapsing experimental autoimmune encephalomyelitis (EAE) using an encephalitogenic epitope of proteolipid protein. J. Neuroimmunol. 38, 229-40 (1992).

21. Pöllinger, B. et al. Spontaneous relapsing-remitting EAE in the SJL/J mouse: MOG-reactive transgenic T cells recruit endogenous MOG-specific B cells. J. Exp. Med. 206, 1303-1316 (2009).

22. Storch, M. K. et al. Cortical Demyelination Can Be Modeled in Specific Rat Models of Autoimmune Encephalomyelitis and Is Major Histocompatability Complex (MHC) Haplotype-Related. J. Neuropathol. Exp. Neurol. 65, 1137-1142 (2006). 
23. Stadelmann, C., Albert, M., Wegner, C. \& Brück, W. Cortical pathology in multiple sclerosis. Curr. Opin. Neurol. 21, 229-234 (2008).

24. Lodygin, D. et al. $\beta$-Synuclein-reactive T cells induce autoimmune CNS grey matter degeneration. Nature 566, 503-508 (2019).

25. Freedman, M. S. et al. Recommended Standard of Cerebrospinal Fluid Analysis in the Diagnosis of Multiple Sclerosis. Arch. Neurol. 62, 865-70 (2005).

26. Siritho, S. \& Freedman, M. S. The prognostic significance of cerebrospinal fluid in multiple sclerosis. J. Neurol. Sci. 279, 21-25 (2009).

27. Hauser, S. L. et al. B-Cell Depletion with Rituximab in Relapsing-Remitting Multiple Sclerosis. N. Engl. J. Med. 358, 676-688 (2008).

28. Golay, J. et al. Lessons for the clinic from rituximab pharmacokinetics and pharmacodynamics. MAbs 5, 826-37 (2013).

29. Keegan, M. et al. Relation between humoral pathological changes in multiple sclerosis and response to therapeutic plasma exchange. Lancet 366, 579-582 (2005).

30. Jarius, S. et al. Screening for MOG-IgG and 27 other anti-glial and anti-neuronal autoantibodies in 'pattern II multiple sclerosis' and brain biopsy findings in a MOG-IgG-positive case. Mult. Scler. J. 22, 1541-1549 (2016).

31. Serafini, B., Rosicarelli, B., Magliozzi, R., Stigliano, E. \& Aloisi, F. Detection of ectopic B-cell follicles with germinal centers in the meninges of patients with secondary progressive multiple sclerosis. Brain Pathol. 14, 164-74 (2004).

32. Litzenburger, T. et al. B Lymphocytes Producing Demyelinating Autoantibodies: Development and Function in Gene-targeted Transgenic Mice. J. Exp. Med. 188, 169-180 (1998).

33. Bettelli, E. et al. Myelin Oligodendrocyte Glycoprotein-specific T Cell Receptor Transgenic Mice Develop Spontaneous Autoimmune Optic Neuritis. J. Exp. Med. 197, 1073-1081 (2003).

34. Molnarfi, N. et al. MHC class II-dependent B cell APC function is required for induction of CNS autoimmunity independent of myelin-specific antibodies. J. Exp. Med. 210, 2921-2937 (2013).

35. Barr, T. A. et al. B cell depletion therapy ameliorates autoimmune disease through ablation of IL-6producing B cells. J. Exp. Med. 209, 1001-1010 (2012).

36. Shen, P. et al. IL-35-producing B cells are critical regulators of immunity during autoimmune and infectious diseases. Nature 507, 366-370 (2014).

37. Yoshizaki, A. et al. Regulatory B cells control T-cell autoimmunity through IL-21-dependent cognate interactions. Nature 491, 264-268 (2012).

38. Linington, C., Bradl, M., Lassmann, H., Brunner, C. \& Vass, K. Augmentation of demyelination in rat acute allergic encephalomyelitis by circulating mouse monoclonal antibodies directed against a myelin/oligodendrocyte glycoprotein. Am. J. Pathol. 130, 443-54 (1988).

39. Nutt, S. L., Hodgkin, P. D., Tarlinton, D. M. \& Corcoran, L. M. The generation of antibody-secreting plasma cells. Nat. Rev. Immunol. 15, 160-171 (2015).

40. Adler, L. N. et al. The Other Function: Class II-Restricted Antigen Presentation by B Cells. Front. Immunol. 8, 319 (2017).

41. Parker, D. C. T Cell-Dependent B Cell Activation. Annu. Rev. Immunol. 11, 331-360 (1993).

42. Kinoshita, K. \& Honjo, T. Linking class-switch recombination with somatic hypermutation. Nat. Rev. Mol. Cell Biol. 2, 493-503 (2001).

43. Stavnezer, J. \& Schrader, C. E. IgH Chain Class Switch Recombination: Mechanism and Regulation. J. Immunol. 193, 5370-5378 (2014).

44. Xu, Z., Zan, H., Pone, E. J., Mai, T. \& Casali, P. Immunoglobulin class-switch DNA recombination: induction, targeting and beyond. Nat. Rev. Immunol. 12, 517-531 (2012).

45. Cheong, T.-C., Compagno, M. \& Chiarle, R. Editing of mouse and human immunoglobulin genes by CRISPR-Cas9 system. Nat. Commun. 7, 10934 (2016).

46. Geha, R. S., Jabara, H. H. \& Brodeur, S. R. The regulation of immunoglobulin E class-switch recombination. Nat. Rev. Immunol. 3, 721-732 (2003).

47. Cerutti, A. The regulation of IgA class switching. Nat. Rev. Immunol. 8, 421-34 (2008). 
48. Rosati, S., Yang, Y., Barendregt, A. \& Heck, A. J. R. Detailed mass analysis of structural heterogeneity in monoclonal antibodies using native mass spectrometry. Nat. Protoc. 9, 967-976 (2014).

49. Schroeder, H. W., Cavacini, L. \& Cavacini, L. Structure and function of immunoglobulins. J. Allergy Clin. Immunol. 125, S41-52 (2010).

50. Klein-Schneegans, A. S., Kuntz, L., Fonteneau, P. \& Loor, F. Serum concentrations of IgM, IgG1, IgG2b, IgG3 and IgA in C57BL/6 mice and their congenics at the Ipr (lymphoproliferation) locus. J.

Autoimmun. 2, 869-75 (1989).

51. Boes, M. Role of natural and immune IgM antibodies in immune responses. Mol. Immunol. 37, 11411149 (2000).

52. Klimovich, V. B. IgM and its receptors: structural and functional aspects. Biochemistry. (Mosc). 76, 534-549 (2011).

53. Ehrenstein, M. R. \& Notley, C. A. The importance of natural IgM: scavenger, protector and regulator. Nat. Rev. Immunol. 10, 778-786 (2010).

54. Heyman, B., Pilström, L. \& Shulman, M. J. Complement activation is required for IgM-mediated enhancement of the antibody response. J. Exp. Med. 167, 1999-2004 (1988).

55. Liu, J. et al. Role of the IgM Fc Receptor in Immunity and Tolerance. Front. Immunol. 10, 529 (2019).

56. Kubagawa, H. et al. Functional Roles of the IgM Fc Receptor in the Immune System. Front. Immunol. 10, 945 (2019).

57. Kinet, J.-P. \& Launay, P. Fc $\alpha / \mu R$ : single member or first born in the family? Nat. Immunol. 1, 371-372 (2000).

58. Nguyen, T. T. T. et al. The IgM receptor $\mathrm{F} c \mu \mathrm{R}$ limits tonic $\mathrm{BCR}$ signaling by regulating expression of the IgM BCR. Nat. Immunol. 18, 321-333 (2017).

59. Preud'homme, J.-L., Petit, I., Barra, A., Morel, Jean-Claude Lecron, F. \& Lelièvre, E. Structural and functional properties of membrane and secreted IgD. Mol. Immunol. 37, 871-887 (2000).

60. Swenson, C. D., Amin, A. R., Xue, B. \& Thorbecke, G. J. Regulation of IgD-Receptor Expression on Murine T Cells: I. Characterization and Metabolic Requirements of the Process Leading to Their Expression. Cell. Immunol. 152, 405-421 (1993).

61. Shan, M. et al. Secreted IgD Amplifies Humoral T Helper 2 Cell Responses by Binding Basophils via Galectin-9 and CD44. Immunity 49, 709-724.e8 (2018).

62. Vieira, P. \& Rajewsky, K. The half-lives of serum immunoglobulins in adult mice. Eur. J. Immunol. 18, 313-316 (1988).

63. Qiao, S.-W. et al. Dependence of antibody-mediated presentation of antigen on FcRn. Proc. Natl. Acad. Sci. 105, 9337-9342 (2008).

64. Leatherbarrow, R. J. \& Dwek, R. A. Binding of complement subcomponent C1q to mouse IgG1, IgG2a and IgG2b: a novel C1q binding assay. Mol. Immunol. 21, 321-7 (1984).

65. Nimmerjahn, F. \& Ravetch, J. V. Divergent immunoglobulin g subclass activity through selective Fc receptor binding. Science 310, 1510-2 (2005).

66. Nimmerjahn, F. \& Ravetch, J. V. Fcy receptors as regulators of immune responses. Nat. Rev. Immunol. 8, 34-47 (2008).

67. Guilliams, M., Bruhns, P., Saeys, Y., Hammad, H. \& Lambrecht, B. N. The function of Fcgamma receptors in dendritic cells and macrophages. Nat. Rev. Immunol. 14, 94-108 (2014).

68. Stone, K. D., Prussin, C. \& Metcalfe, D. D. IgE, mast cells, basophils, and eosinophils. J. Allergy Clin. Immunol. 125, S73-80 (2010).

69. Motran, C. C. et al. Helminth Infections: Recognition and Modulation of the Immune Response by Innate Immune Cells. Front. Immunol. 9, 664 (2018).

70. Ettinger, R. et al. Pathogenic mechanisms of IgE-mediated inflammation in self-destructive autoimmune responses. Autoimmunity 50, 25-36 (2017).

71. Kehry, M. R. \& Yamashita, L. C. Low-affinity IgE receptor (CD23) function on mouse B cells: role in IgEdependent antigen focusing. Proc. Natl. Acad. Sci. U. S. A. 86, 7556-60 (1989).

72. $\mathrm{Li}, \mathrm{X} . \&$ Kimberly, R. P. Targeting the Fc receptor in autoimmune disease. Expert Opin. Ther. Targets 18, 335-50 (2014). 
73. Roos, A. et al. Human IgA Activates the Complement System Via the Mannan-Binding Lectin Pathway. J. Immunol. 167, 2861-2868 (2001).

74. Stad, R. K., van Gijlswijk-Janssen, D. J., van Es, L. A. \& Daha, M. R. Complement depletion abolishes IgA-mediated glomerular inflammation in rats. Exp. Nephrol. 2, 182-9

75. Pabst, O. New concepts in the generation and functions of IgA. Nat. Rev. Immunol. 12, 821-832 (2012).

76. Gommerman, J. L., Rojas, O. L. \& Fritz, J. H. Re-thinking the functions of IgA ${ }^{+}$plasma cells. Gut Microbes 5, 652-662 (2014).

77. Bournazos, S., Woof, J. M., Hart, S. P. \& Dransfield, I. Functional and clinical consequences of Fc receptor polymorphic and copy number variants. Clin. Exp. Immunol. 157, 244-254 (2009).

78. Woof, J. M. \& Russell, M. W. Structure and function relationships in IgA. Mucosal Immunol. 4, 590597 (2011).

79. Willis, S. N. et al. Investigating the Antigen Specificity of Multiple Sclerosis Central Nervous SystemDerived Immunoglobulins. Front. Immunol. 6, 600 (2015).

80. Brändle, S. M. et al. Distinct oligoclonal band antibodies in multiple sclerosis recognize ubiquitous self-proteins. Proc. Natl. Acad. Sci. 113, 7864-7869 (2016).

81. Krumbholz, M. \& Meinl, E. B cells in MS and NMO: pathogenesis and therapy. Semin. Immunopathol. 36, 339-350 (2014).

82. Pham-Dinh, D. et al. Myelin/oligodendrocyte glycoprotein is a member of a subset of the immunoglobulin superfamily encoded within the major histocompatibility complex. Proc. Natl. Acad. Sci. U. S. A. 90, 7990-4 (1993).

83. Elliott, C. et al. Functional identification of pathogenic autoantibody responses in patients with multiple sclerosis. Brain 135, 1819-1833 (2012).

84. Piddlesden, S. J., Lassmann, H., Zimprich, F., Morgan, B. P. \& Linington, C. The demyelinating potential of antibodies to myelin oligodendrocyte glycoprotein is related to their ability to fix complement. $J$. Neuroimmunol. 35, 111 (2003).

85. Weber, M. S., Hemmer, B. \& Cepok, S. The role of antibodies in multiple sclerosis. Biochim. Biophys. Acta - Mol. Basis Dis. 1812, 239-245 (2011).

86. Kinzel, S. et al. Myelin-reactive antibodies initiate T cell-mediated CNS autoimmune disease by opsonization of endogenous antigen. Acta Neuropathol. 132, 43-58 (2016).

87. Flach, A.-C. et al. Autoantibody-boosted T-cell reactivation in the target organ triggers manifestation of autoimmune CNS disease. Proc. Natl. Acad. Sci. 113, 3323-3328 (2016).

88. Abdul-Majid, K.-B. et al. Fc Receptors are Critical for Autoimmune Inflammatory Damage to the Central Nervous System in Experimental Autoimmune Encephalomyelitis. Scand. J. Immunol. 55, 7081 (2002).

89. Duncan, A. R. \& Winter, G. The binding site for C1q on IgG. Nature 332, 738-740 (1988).

90. Mihai, S. et al. Specific Inhibition of Complement Activation Significantly Ameliorates Autoimmune Blistering Disease in Mice. Front. Immunol. 9, 535 (2018).

91. Ran, F. A. et al. Genome engineering using the CRISPR-Cas9 system. Nat. Protoc. 8, 2281-2308 (2013).

92. Linington, C., Bradl, M., Lassmann, H., Brunner, C. \& Vass, K. Augmentation of demyelination in rat acute allergic encephalomyelitis by circulating mouse monoclonal antibodies directed against a myelin/oligodendrocyte glycoprotein. Am. J. Pathol. 130, 443-54 (1988).

93. LAEMMLI, U. K. Cleavage of Structural Proteins during the Assembly of the Head of Bacteriophage T4. Nature 227, 680-685 (1970).

94. Burnette, W. N. "Western Blotting": Electrophoretic transfer of proteins from sodium dodecyl sulfatepolyacrylamide gels to unmodified nitrocellulose and radiographic detection with antibody and radioiodinated protein A. Anal. Biochem. 112, 195-203 (1981).

95. Takai, T., Ono, M., Hikida, M., Ohmori, H. \& Ravetch, J. V. Augmented humoral and anaphylactic responses in FcyRII-deficient mice. Nature 379, 346-349 (1996).

96. Takai, T., Li, M., Sylvestre, D., Clynes, R. \& Ravetch, J. V. FcR gamma chain deletion results in pleiotrophic effector cell defects. Cell 76, 519-529 (1994). 
97. Roopenian, D. C. et al. The MHC Class I-Like IgG Receptor Controls Perinatal IgG Transport, IgG Homeostasis, and Fate of IgG-Fc-Coupled Drugs. J. Immunol. 170, 3528-3533 (2003).

98. Barnes, N. et al. FcgammaRI-deficient mice show multiple alterations to inflammatory and immune responses. Immunity 16, 379-89 (2002).

99. Hazenbos, W. L. et al. Impaired IgG-dependent anaphylaxis and Arthus reaction in Fc gamma RIII (CD16) deficient mice. Immunity 5, 181-8 (1996).

100. Nimmerjahn, F., Bruhns, P., Horiuchi, K. \& Ravetch, J. V. FcyRIV: A Novel FcR with Distinct IgG Subclass Specificity. Immunity 23, 41-51 (2005).

101. Moran, A. E. et al. T cell receptor signal strength in $\mathrm{T}_{\text {reg }}$ and iNKT cell development demonstrated by a novel fluorescent reporter mouse. J. Exp. Med. 208, 1279-1289 (2011).

102. Klüver, H. \& Barrera, E. A Method for the Combined Staining of Cells and Fibers in the Nervous System. J. Neuropathol. Exp. Neurol. 12, 400-403 (1953).

103. Jennewein, M. F. \& Alter, G. The Immunoregulatory Roles of Antibody Glycosylation. Trends Immunol. 38, 358-372 (2017).

104. Brehm, U., Piddlesden, S. J., Gardinier, M. V \& Linington, C. Epitope specificity of demyelinating monoclonal autoantibodies directed against the human myelin oligodendrocyte glycoprotein (MOG). J. Neuroimmunol. 97, 9-15 (1999).

105. Breithaupt, C. et al. Demyelinating Myelin Oligodendrocyte Glycoprotein-Specific Autoantibody Response Is Focused on One Dominant Conformational Epitope Region in Rodents. J. Immunol. 181, 1255-1263 (2008).

106. Faiß, L. Isotype switching of a MOG-specific hybridoma using the CRISPR Cas 9 system. (Göttingen university, 2018).

107. Brezski, R. J. \& Georgiou, G. Immunoglobulin isotype knowledge and application to Fc engineering. Curr. Opin. Immunol. 40, 62-9 (2016).

108. Janda, A., Bowen, A., Greenspan, N. S. \& Casadevall, A. Ig constant region effects on variable region structure and function. Front. Microbiol. 7, 1-10 (2016).

109. Strauß, J. Elucidating the mechanisms of disease-triggering myelin-specific autoantibodies. (Göttingen University, 2019).

110. Peschl, P. et al. Human antibodies against the myelin oligodendrocyte glycoprotein can cause complement-dependent demyelination. J. Neuroinflammation 14, 208 (2017).

111. Stadelmann, C., Wegner, C. \& Brück, W. Inflammation, demyelination, and degeneration - Recent insights from MS pathology. Biochimica et Biophysica Acta - Molecular Basis of Disease (2011). doi:10.1016/j.bbadis.2010.07.007

112. Kurschus, F. T cell mediated pathogenesis in EAE: Molecular mechanisms. Biomed. J. 38, 183 (2015).

113. Ober, R. J., Martinez, C., Vaccaro, C., Zhou, J. \& Ward, E. S. Visualizing the site and dynamics of IgG salvage by the MHC class I-related receptor, FcRn. J. Immunol. 172, 2021-9 (2004).

114. van de Bovenkamp, F. S., Hafkenscheid, L., Rispens, T. \& Rombouts, Y. The Emerging Importance of IgG Fab Glycosylation in Immunity. J. Immunol. 196, 1435-1441 (2016).

115. Hemmer, B., Kerschensteiner, M. \& Korn, T. Role of the innate and adaptive immune responses in the course of multiple sclerosis. Lancet Neurol. 14, 406-419 (2015).

116. Steinman, L. Immunology of Relapse and Remission in Multiple Sclerosis. Annu. Rev. Immunol. 32, 257-281 (2014).

117. Capecchi, M. R. Gene targeting in mice: functional analysis of the mammalian genome for the twentyfirst century. Nat. Rev. Genet. 6, 507-512 (2005).

118. Petrezselyova, S. et al. Homology arms of targeting vectors for gene insertions and CRISPR/Cas9 technology: size does not matter; quality control of targeted clones does. Cell. Mol. Biol. Lett. 20, 773-87 (2015).

119. Suzuki, K. et al. In vivo genome editing via CRISPR/Cas9 mediated homology-independent targeted integration. Nature 540, 144-149 (2016). 
120. Schluesener, H. J., Sobel, R. A., Linington, C. \& Weiner, H. L. A monoclonal antibody against a myelin oligodendrocyte glycoprotein induces relapses and demyelination in central nervous system autoimmune disease. J. Immunol. 139, 4016-21 (1987).

121. Bettelli, E., Baeten, D., Jäger, A., Sobel, R. A. \& Kuchroo, V. K. Myelin oligodendrocyte glycoproteinspecific T and B cells cooperate to induce a Devic-like disease in mice. J. Clin. Invest. 116, 2393-2402 (2006).

122. Collins, A. M. IgG subclass co-expression brings harmony to the quartet model of murine IgG function. Immunol. Cell Biol. 94, 949-954 (2016).

123. Díaz de Ståhl, T., Dahlstrom, J., Carroll, M. C. \& Heyman, B. A role for complement in feedback enhancement of antibody responses by IgG3. J. Exp. Med. 197, 1183-90 (2003).

124. Abdalla, A. O. et al. Kinetics of Cytokine Gene Expression in Human CD4+ and CD8+ T-Lymphocyte Subsets Using Quantitative Real-Time PCR. Scand. J. Immunol. 58, 601-606 (2003).

125. Pala, P., Verhoef, A., Lamb, J. R. \& Openshaw, P. J. M. Single cell analysis of cytokine expression kinetics by human CD4+ T-cell clones during activation or tolerance induction. Immunology 100, 209216 (2000).

126. SARENEVA, T. et al. Kinetics of cytokine and NFAT gene expression in human interleukin-2-dependent T lymphoblasts stimulated via T-cell receptor. Immunology 93, 350-357 (2001).

127. Lawrence, M. G. et al. Half-life of IgE in serum and skin: Consequences for anti-IgE therapy in patients with allergic disease. J. Allergy Clin. Immunol. 139, 422-428.e4 (2017).

128. Acharya, M. et al. CD23/FceRII: molecular multi-tasking. Clin. Exp. Immunol. 162, 12-23 (2010).

129. Sutton, B. J. \& Davies, A. M. Structure and dynamics of IgE-receptor interactions: FceRI and CD23/FcERII. Immunol. Rev. 268, 222-235 (2015).

130. Poole, J. A., Meng, J., Cao, L., Bates, C. \& Rosenwasser, L. J. The low affinity IgE receptor, CD23, expressed on antigen presenting cells alters T-cell response to allergen. J. Allergy Clin. Immunol. 113, S210 (2004).

131. Brown, M. A. \& Weinberg, R. B. Mast Cells and Innate Lymphoid Cells: Underappreciated Players in CNS Autoimmune Demyelinating Disease. Front. Immunol. 9, 514 (2018).

132. Lock, C. et al. Gene-microarray analysis of multiple sclerosis lesions yields new targets validated in autoimmune encephalomyelitis. Nat. Med. 8, 500-508 (2002).

133. Brown, M. A., Tanzola, M. B. \& Robbie-Ryan, M. Mechanisms underlying mast cell influence on EAE disease course. Mol. Immunol. 38, 1373-8 (2002).

134. Secor, V. H., Secor, W. E., Gutekunst, C.-A. \& Brown, M. A. Mast Cells Are Essential for Early Onset and Severe Disease in a Murine Model of Multiple Sclerosis. J. Exp. Med. 191, 813-822 (2000).

135. Feyerabend, T. B. et al. Cre-Mediated Cell Ablation Contests Mast Cell Contribution in Models of Antibody- and T Cell-Mediated Autoimmunity. Immunity 35, 832-844 (2011).

136. Musio, S. et al. Treatment with anti-FceRla antibody exacerbates EAE and T-cell immunity against myelin. Neurol. Neuroimmunol. Neurolnflammation 4, (2017).

137. Kinet, J. P. \& Launay, P. Fc alpha/microR: single member or first born in the family? Nature immunology 1, 371-372 (2000).

138. Rojas, O. L. et al. Recirculating Intestinal IgA-Producing Cells Regulate Neuroinflammation via IL-10. Cell 176, 610-624.e18 (2019).

139. Schumacher, H. et al. IgA autoantibodies against native myelin basic protein in a patient with MS. Neurol. - Neuroimmunol. Neuroinflammation 6, e569 (2019).

140. van den Hoogen, W. J., Laman, J. D. \& 't Hart, B. A. Modulation of Multiple Sclerosis and Its Animal Model Experimental Autoimmune Encephalomyelitis by Food and Gut Microbiota. Front. Immunol. 8, 1081 (2017).

141. Pröbstel, A.-K. \& Baranzini, S. E. The Role of the Gut Microbiome in Multiple Sclerosis Risk and Progression: Towards Characterization of the "MS Microbiome". Neurotherapeutics 15, 126-134 (2018).

142. Kim, M. \& Kim, C. H. Regulation of humoral immunity by gut microbial products. Gut Microbes 8, 392 399 (2017). 
143. Litinskiy, M. B. et al. DCs induce CD40-independent immunoglobulin class switching through BLyS and APRIL. Nat. Immunol. 3, 822-829 (2002).

144. Singh, K., Chang, C. \& Gershwin, M. E. IgA deficiency and autoimmunity. Autoimmun. Rev. 13, 163177 (2014).

145. Willenborg, D. O. \& Prowse, S. J. Immunoglobulin-deficient rats fail to develop experimental allergic encephalomyelitis. J. Neuroimmunol. 5, 99-109 (1983).

146. WILLENBORG, D., SJOLLEMA, P. \& DANTA, G. Immunoglobulin deficient rats as donors and recipients of effector cells of allergic encephalomyelitis. J. Neuroimmunol. 11, 93-103 (1986).

147. Nguyen, T. T. T., Elsner, R. A. \& Baumgarth, N. Natural IgM prevents autoimmunity by enforcing B cell central tolerance induction. J. Immunol. 194, 1489-1502 (2015).

148. Nguyen, T. T. T. \& Baumgarth, N. Natural IgM and the Development of B Cell-Mediated Autoimmune Diseases. Crit. Rev. Immunol. 36, 163-177 (2016).

149. Ouchida, R. et al. Critical role of the IgM Fc receptor in IgM homeostasis, B-cell survival, and humoral immune responses. Proc. Natl. Acad. Sci. 109, E2699-E2706 (2012).

150. Pedotti, R. et al. Multiple elements of the allergic arm of the immune response modulate autoimmune demyelination. Proc. Natl. Acad. Sci. 100, 1867-1872 (2003).

151. Szalai, A. J., Hu, X., Raman, C. \& Barnum, S. R. Requirement of the Fc receptor common $\gamma$-chain fory $\delta$ T cell-mediated promotion of murine experimental autoimmune encephalomyelitis. Eur. J. Immunol. 35, 3487-3492 (2005).

152. Breij, E. C. W. et al. The FcRy Chain Is Not Essential for Induction of Experimental Allergic Encephalomyelitis (EAE) or Anti-Myelin Antibody-Mediated Exacerbation of EAE. J. Neuropathol. Exp. Neurol. 64, 304-311 (2005).

153. Piddlesden, S. J., Lassmann, H., Zimprich, F., Morgan, B. P. \& Linington, C. The demyelinating potential of antibodies to myelin oligodendrocyte glycoprotein is related to their ability to fix complement. Am. J. Pathol. 143, 555-64 (1993).

154. Wills-Karp, M. Complement activation pathways: a bridge between innate and adaptive immune responses in asthma. Proc. Am. Thorac. Soc. 4, 247-51 (2007).

155. Costanza, M., Colombo, M. P. \& Pedotti, R. Mast cells in the pathogenesis of multiple sclerosis and experimental autoimmune encephalomyelitis. Int. J. Mol. Sci. 13, 15107-15125 (2012).

156. Johnson, D., Seeldrayers, P. A. \& Weiner, H. L. The role of mast cells in demyelination. 1. Myelin proteins are degraded by mast cell proteases and myelin basic protein and $\mathrm{P} 2$ can stimulate mast cell degranulation. Brain Res. 444, 195-198 (1988).

157. MacGlashan, D. IgE receptor and signal transduction in mast cells and basophils. Curr. Opin. Immunol. 20, 717-723 (2008).

158. Nataf, S., Carroll, S. L., Wetsel, R. A., Szalai, A. J. \& Barnum, S. R. Attenuation of Experimental Autoimmune Demyelination in Complement-Deficient Mice. J. Immunol. 165, 5867-5873 (2000).

159. Heyman, B., Andrighetto, G. \& Wigzell, H. Antigen-dependent IgM-mediated enhancement of the sheep erythrocyte response in mice. Evidence for induction of B cells with specificities other than that of the injected antibodies. J. Exp. Med. 155, 994-1009 (1982).

160. Sharp, T. H. et al. Insights into IgM-mediated complement activation based on in situ structures of IgM-C1-C4b. Proc. Natl. Acad. Sci. 116, 201901841 (2019).

161. Ding, Z. et al. Complement-activating IgM enhances the humoral but not the $T$ cell immune response in mice. PLoS One 8, e81299 (2013).

162. Zhang, L., Ding, Z. \& Heyman, B. IgG3-antigen complexes are deposited on follicular dendritic cells in the presence of C1q and C3. Sci. Rep. 7, 5400 (2017).

163. Ozakbas, S., Cinar, B. P., Özcelik, P., Baser, H. \& Kosehasanoğullari, G. Intrathecal IgM index correlates with a severe disease course in multiple sclerosis: Clinical and MRI results. Clin. Neurol. Neurosurg. 160, 27-29 (2017).

164. Urich, E., Gutcher, I., Prinz, M. \& Becher, B. Autoantibody-mediated demyelination depends on complement activation but not activatory Fc-receptors. Proc. Natl. Acad. Sci. 103, 18697-18702 (2006). 
165. Klaus, G. G., Pepys, M. B., Kitajima, K. \& Askonas, B. A. Activation of mouse complement by different classes of mouse antibody. Immunology 38, 687 (1979).

166. Anthony, R. M. \& Nimmerjahn, F. The role of differential IgG glycosylation in the interaction of antibodies with FcyRs in vivo. Curr. Opin. Organ Transplant. 16, 7-14 (2011).

167. Chung, A. W. et al. Identification of antibody glycosylation structures that predict monoclonal antibody Fc-effector function. AIDS 28, 2523-30 (2014).

168. McLean, G. R., Torres, M., Elguezabal, N., Nakouzi, A. \& Casadevall, A. Isotype Can Affect the Fine Specificity of an Antibody for a Polysaccharide Antigen. J. Immunol. 169, 1379-1386 (2002).

169. Casadevall, A. \& Janda, A. Immunoglobulin isotype influences affinity and specificity. Proc. Natl. Acad. Sci. 109, 12272-12273 (2012).

170. Wu, Y., Zhong, L. \& Geng, J. Neuromyelitis optica spectrum disorder: Pathogenesis, treatment, and experimental models. Mult. Scler. Relat. Disord. 27, 412-418 (2019).

171. Wines, B. D., Powell, M. S., Parren, P. W. H. I., Barnes, N. \& Hogarth, P. M. The IgG Fc Contains Distinct Fc Receptor (FcR) Binding Sites: The Leukocyte Receptors FcyRI and FcyRlla Bind to a Region in the Fc Distinct from That Recognized by Neonatal FcR and Protein A. J. Immunol. 164, 5313-5318 (2000).

172. Lund, J. et al. Multiple binding sites on the $\mathrm{CH} 2$ domain of IgG for mouse Fc gamma R11. Mol. Immunol. 29, 53-9 (1992).

173. Saunders, K. O. Conceptual Approaches to Modulating Antibody Effector Functions and Circulation Half-Life. Front. Immunol. 10, 1296 (2019). 


\section{Curriculum Vitae}

Personal data

Name

Address

Phone

E-mail

2015-2016

2013-2015

2010-2013

\section{César Cordero Gómez}

Institute for Neuroimmunology \& Multiple Sclerosis Research (IMSF)

Von Siebold-Straße 3A

37075 Göttingen

+49 17674687556

ccorgomez@gmail.com

Academic Education

PhD student at the Institute for Neuroimmunology \& Multiple Sclerosis Research (IMSF) in Göttingen, Germany.

Research assistant at the institute for Neuroimmunology \& Multiple Sclerosis Research (IMSF) in Göttingen, Germany.

Master in Neuroscience. Autonomous University of Madrid (UAM), Spain.

Master Thesis - "Role of the endocannabinoid 2-AG on inflammation in the acute phase of the infection with Theiler's murine encephalomyelitis virus" at Cajal Institute's neuroimmunology research group in Madrid, Spain.

Academic studies in Biomedical Sciences. Autonomous University of Barcelona (UAB), Spain.

Research associate as Transmission Electron Microscope technician at "BBG - Glial Research Team", Unit of Histology, Department of Cell Biology, Physiology and Immunology, Faculty of Medicine, Autonomous University of Barcelona (UAB), Spain. 


\section{Publications}

Flach AC, Litke T, Strauss J, Haberl M, Gómez CC, Reindl M, Saiz A, Fehling HJ, Wienands J, Odoardi F, Lühder F, Flügel A. (2016) Autoantibody-boosted T-cell reactivation in the target organ triggers manifestation of autoimmune CNS disease. Proc Natl Acad Sci U S A doi: 10.1073/pnas.1519608113

Miriam Mecha, Ana Feliú, Isabel Machín, Cesar Cordero, Francisco Carrillo-Salinas, Leyre Mestre, Gloria Hernández-Torres, Silvia Ortega-Gutiérrez, María L López-Rodríguez, Fernando de Castro, Diego Clemente, Carmen Guaza. (2018) 2-AG limits Theiler's virus induced acute neuroinflammation by modulating microglia and promoting MDSCs. Glia 10.1002/glia.23317 